\title{
Normally hyperbolic operators, the Huygens property and conformal geometry
}

\author{
H. Baum \\ I. Kath
}

Sfb 288 Preprint No. 212

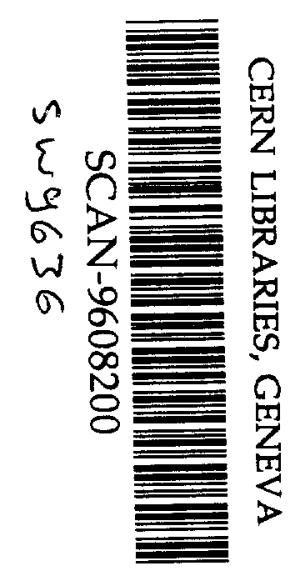

Diese Arbeit ist mit Unterstützung des von der Deutschen Forschungsgemeinschaft getragenen Sonderforschungsbereiches 288 entstanden und als Manuskript vervielfältigt worden.

Berlin, Juli 1996

The list of preprints of the Sonderforschungsbereich 288 is available at: http://www-sfb288. math.tu-berlin.de 


\title{
Normally hyperbolic operators, the Huygens property and conformal geometry
}

\author{
Helga Baum and Ines Kath
}

July 12,1996

In memory of Paul Günther

\begin{abstract}
In this paper we give a review on normally hyperbolic operators of Huygens type. The methods to determine Huygens operators we explain here were essentially influenced and developed by Paul Günther.
\end{abstract}

\section{Contents}

1 Introduction 2

2 Riesz distributions 4

2.1 Riesz distributions on Minkowski spaces . . . . . . . . . . . . 4

2.2 Riesz distributions on geodesically normal domains in Lorentzian manifolds . . . . . . . . . . . . . . . . . . . 6

3 Normally hyperbolic operators $\quad \mathbf{8}$

3.1 Definition and examples . . . . . . . . . . . . . . . 8

3.2 The Weitzenböck formula . . . . . . . . . . . . . . . . 9

3.3 Hadamard coefficients . . . . . . . . . . . . . . . . 10

3.4 Fundamental solution $\ldots \ldots \ldots \ldots \ldots$

4 Huygens operators $\quad 14$

5 Conformal gauge invariance of Huygens operators $\quad 15$

6 The moments of normally hyperbolic operators 18

6.1 Mappings with simple transformation law . . . . . . . . . . 18

6.2 The conformal gauge derivative . . . . . . . . . . . 20

6.3 The trace free part of mappings with simple transformation law . . . . 26

6.4 Kernels with simple transformation law . . . . . . . . . . . 27

6.5 Construction of the moments . . . . . . . . . . . 33

6.6 Moments of small order . . . . . . . . . . . . 36 
7 Applications $\quad \mathbf{5 7}$

7.1 Dirac operators . . . . . . . . . . . . . . . . 57

7.2 Hodge-Laplace operators . . . . . . . . . . . . . . . . . . . . 59

7.3 The Yamabe operator . . . . . . . . . . . . . . . . . . 59

7.4 The wave operator of Riemannian symmetric spaces . . . . . . . 60

7.5 The D'Alambert operator plus potentials on the Minkowski space . . . . 61

\section{Introduction}

It is a familiar phenomenon of daily life that waves propagate quite differently in 2 and 3 dimensions. When a pebble falls into water at a certain point $x_{0}$, circular waves around $x_{0}$ are formed. A given point near $x_{0}$ will be hit by an initial ripple and later by residual waves. 3-dimensionally, the situation is quite different. If we produce a sound localized at the neighbourhood of a point $x_{0}$ then someone near $x_{0}$ will hear the sound during a certain time interval but no longer. There are no residual waves like those present on the water surface; thus music can exist in $\mathbb{R}^{3}$ but not in $\mathbb{R}^{2}$. The mathematical reason for this different behaviour is a special property of the fundamental solution of the wave operator $\square_{m}$ of the $\mathbb{R}^{m}$ in dimension $m=3$. Whereas in general the forward fundamental solution of $\square_{m}$ with respect to the point $o \in \mathbb{R}^{m+1}$ is supported in the future cone $\mathcal{J}_{+}(o)=\left\{(x, t) \in \mathbb{R}^{m} \times \mathbb{R} \mid\|x\| \leq t\right\}$ the forward fundamental solution in dimension $m=3$ and each other odd dimension $m \geq 3$ is supported even in the light cone $\mathcal{C}_{+}(o)=\left\{(x, t) \in \mathbb{R}^{m} \times \mathbb{R} \mid\|x\|=t\right\}$. This produces a "sharp" wave propagation. Operators describing a "sharp" wave propagation such as $\square_{3}$ are called operators of Huygens type or shortly Huygens operators. In 1923, in his Yale Lectures, J. Hadamard posed the problem of finding all normally hyperbolic operators of Huygens type (see [Had23], p.236). In spite of its age this problem is still far from being completely solved.

Already in the beginning of his mathernatical work P. Günther dealt with Huygens operators. He found the first geometric conditions for normally hyperbolic operators acting on functions of a 4-dimensional manifold to be of Huygens type [Gün52]. Furthermore he found the first examples of nontrivial Huygens operators on 4-dimensional manifolds (see [Gün65]). Later on he developed in collaboration with V. Wünsch and R. Schimming the idea to characterize the Huygens property by a sequence of conformal invariants, the so-called moments of a normally hyperbolic operator (see [Wün76], [Sch78], [Sch84], [GW85], [GW86], [Gün88]).

Let us briefly define Huygens operators and describe the methods to determine operators of Huygens type. An operator $P$ acting on a vector bundle $E$ over an $n$ dimensional manifold $M$ is called normally hyperbolic if its principal symbol is given by a Lorentzian metric $g$. Because of this property the operator $P$ admits a Weitzenböck formula; therefore, there is a uniquely determined covariant derivative $\nabla^{P}$ in $E$ and a homomorphism $H_{P}$ on $E$ belonging to $P$. Each normally hyperbolic operator has uniquely determined local forward and backward fundamental solutions with respect to a point $x$ of a causal domain of $M$. As in the case of the wave operator of the $\mathbb{R}^{m}$ these fundamental solutions are supported in the future and past cone of $x$ in 
general. An operator is called Huygens operator if there exists a covering of $M$ by causal domains such that all forward and backward fundamental solutions with respect to these domains are supported in the forward or backward light cone. Using this property of the fundamental solution it can be proved that Huygens operators are those operators describing a "sharp" wave propagation i.e. an observer receives a signal from a bounded source only during a bounded time interval. The general properties of the fundamental solution show that there are no Huygens operators in odd dimension $n$ or $n=2$; so one has to study the problem only in even dimensions $n \geq 4$.

Using methods of Harmonic Analysis Helgason, Olafsson and Schlichtkrull were able to calculate the fundamental solution of the wave operator of non-compact Riemannian symmetric spaces and determine those Riemannian symmetric spaces whose wave operator is of Huygens type ([Hel92], [Hel94], [OS92]).

Another method to find Huygens operators is given by the Hadamard criterion. With the normally hyperbolic operator $P$ and each geodesic normal domain $\Omega$ of $M$ there is associated a series of smooth sections $U_{k} \in \Gamma\left(\Omega \times \Omega, E^{*} \otimes E\right), k=1,2, \ldots$, the so-called Hadamard coefficients of $P$, defined by a recursive system of differential equations using the covariant derivative on $E$ assigned to $P$ by its Weitzenböck formula. The operator $P$ is of Huygens type if the Hadamard coefficient $U_{\frac{n-2}{2}}$ vanishes on a certain subset of $\Omega \times \Omega$. For special classes of normally hyperbolic operators it was possible to calculate the Hadamard coefficients $U_{\frac{n-2}{2}}$ and to check that they vanish ([Gün65], [Sch71], [BV94a], [BV94b], [Bau96]).

The third method to determine Huygens operators makes use of the conformal gauge invariance of the Huygens property. A normally hyperbolic operator $P$ is of Huygens type if and only if each of its conformal gauge transforms is of Huygens type. Hence, there should be a complete system of conformal gauge invariants which describes the set of Huygens operators in the set of all normally hyperbolic operators acting on a certain vector bundle $E$. Such conformal gauge invariants, the so-called moments of a normally hyperbolic operator, were defined first for special classes of operators in various ways and finally described completely by $\mathrm{P}$. Günther [Gün88]. To define the moments of an operator $P$ one applies a certain differentiation procedure to the tail term of the fundamental solution of $P$. In general a conformal gauge invariant assigned to $P$ after differentiation with respect to the covariant derivative defined by $P$ via its Weitzenböck formula is no longer gauge invariant. Therefore, a new theory of the socalled conformal gauge derivatives was developed. This sort of derivatives respects a slightly larger class of tensors assigned to $P$ than the conformal gauge invariants, namely the tensors with simple transformation law. It turns out that certain combinations of conformal gauge derivatives of the tail term of the fundamental solution of $P$ are conformally gauge invariant. In this way one can assign to each normally hyperbolic operator $P$ acting on a vector bundle $E$ over a manifold $M^{n}$ of even dimension $n \geq 4$ a sequence $I_{k}(P) \in \Gamma\left(S^{k}\left(T^{*} M\right) \otimes \operatorname{Hom}(E, E)\right), k=0,1, \ldots$, of trace free conformal gauge invariants of order $k$ and weight $\omega=1-\frac{n}{2}$, the moments of $P$. If $P$ is of Huygens type, all of its moments $I_{k}(P)$ vanish. If $M$ and $P$ are real analytic the vanishing of all moments is also sufficient for the operator to be of Huygens type. In dimension 
$n=4$ there are explicit formulas which express the first five moments in terms of the curvature of the manifold $M$ and the curvature of the covariant derivative assigned to the operator by its Weitzenböck formula. This provides an effective method to study the Huygens property for operators on 4-dimensional manifolds. In this way, for example, all 4-dimensional Lorentzian manifolds have been found on which the Hodge-Laplace operator on forms and the square of the Dirac operator are of Huygens type and a large class of 4-dimensional manifolds (conjectured to be all) on which the Yamabe operator is Huygens ([McL69], [Sch78], [Wün78], [Wün79], [Wün80], [Wün85], [CM86], [CM87], [CM88], [CCMW91], [AM93], [AM94], [Wün94]).

\section{Riesz distributions}

\subsection{Riesz distributions on Minkowski spaces}

In this chapter we recall some properties of the Riesz distributions which we will need to describe the fundamental solution of a normally hyperbolic operator. The proofs can be found in [Gün88], , chap. 2.

We consider the Minkowski space $\left(\mathbb{R}^{n}, g_{0}\right)$, where $g_{0}$ is the inner product defined by

$$
g_{0}(x, y)=-x_{1} y_{1}+x_{2} y_{2}+\ldots+x_{n} y_{n} .
$$

As usual we set $x=\left(x_{1}, x_{2}, \ldots, x_{n}\right)$ and $y=\left(y_{1}, y_{2}, \ldots, y_{n}\right)$. Let $\sigma(x)$ denote the quadratic distance from the point $x \in \mathbb{R}^{n}$ to $0 \in \mathbb{R}^{n}$, i.e.

$$
\sigma(x):=g_{0}(x, x)=-x_{1}^{2}+x_{2}^{2}+\ldots+x_{n}^{2} .
$$

Then

$$
\mathcal{J}_{+}(0):=\left\{x \in \mathbb{R}^{n} \mid-\sigma(x) \geq 0, x_{1} \geq 0\right\}
$$

is the future of $0 \in \mathbb{R}^{n}$ and

$$
\mathcal{C}_{+}(0):=\partial \mathcal{J}_{+}(0)=\left\{x \in \mathbb{R}^{n} \mid \sigma(x)=0, x_{1} \geq 0\right\}
$$

the future light cone of $0 \in \mathbb{R}^{n}$. Similarly, we define $\mathcal{J}_{-}(0)$ and $\mathcal{C}_{-}(0)$, where now $x_{1} \leq 0$.

Definition 2.1 Let $\alpha \in \mathbb{C}$ be a complex number with Re $(\alpha) \geq n=\operatorname{dim} \mathbb{R}^{n}$. Then we define regular distributions $R_{+}(\alpha)$ and $R_{-}(\alpha)$ on $C_{0}^{\infty}\left(\mathbb{R}^{n}\right)$ by

$$
\left\langle R_{ \pm}(\alpha), \varphi\right\rangle:=\frac{\pi^{\frac{2-n}{2}} 2^{1-\alpha}}{\Gamma\left(\frac{\alpha}{2}\right) \Gamma\left(\frac{\alpha-n}{2}+1\right)} \int_{\mathcal{J}_{ \pm}(0)}(-\sigma(x))^{\frac{\alpha-n}{2}} \varphi(x) \mu_{0}(x)
$$

for all test functions $\varphi \in C_{0}^{\infty}\left(\mathbb{R}^{n}\right)$. Here $\Gamma$ denotes the gamma-function and $\mu_{0}$ the Lebesgue measure.

$R_{+}(\alpha)$ and $R_{-}(\alpha) \in \mathcal{D}^{\prime}\left(\mathbb{R}^{n}\right)$ are called Riesz distributions . 


\section{Remark}

1. For any fixed test function $\varphi$ the function $\left\langle R_{ \pm}(\alpha), \varphi\right\rangle$ is holomorphic in $\alpha$ in $\{\alpha \in \mathbb{C} \mid \operatorname{Re} \alpha>n\}$. We will say that $R_{ \pm}(\cdot)$ are holomorphic distributions in $\{\alpha \in \mathbb{C} \mid \operatorname{Re} \alpha>n\}$.

2. Let $\varphi$ be in $C_{0}^{\infty}\left(\mathbb{R}^{n}\right)$. With $\hat{\varphi}\left(x_{1}, \ldots, x_{n}\right):=\varphi\left(-x_{1}, x_{2}, \ldots, x_{n}\right)$ we have

$$
\left\langle R_{-}(\alpha), \varphi\right\rangle=\left\langle R_{+}(\alpha), \hat{\varphi}\right\rangle .
$$

The Riesz distributions $R_{+}(\alpha)$ have the following properties.

Proposition 2.1 If $\operatorname{Re} \alpha>n$ then

(i) $-\sigma R_{ \pm}(\alpha)=\alpha(\alpha-n+2) R_{ \pm}(\alpha+2)$

(ii) $-\partial_{i} \sigma \cdot R_{ \pm}(\alpha)=2 \alpha \partial_{i} R_{ \pm}(\alpha+2)$

(iii) $R_{ \pm}(\alpha)=\square R_{ \pm}(\alpha+2)$ for $\operatorname{Re} \alpha>n+2$,

where $\square=\left(\partial_{1}\right)^{2}-\left(\partial_{2}\right)^{2}-\ldots-\left(\partial_{n}\right)^{2}$ is the wave operator of $\mathbb{R}^{n}$.

From (iii) of Proposition 2.1 we see that for any test function $\varphi \in C_{0}^{\infty}\left(\mathbb{R}^{n}\right)$ the function $\left\langle R_{+}(\cdot), \varphi\right\rangle$ admits a holomorphic extension to $\mathbb{C}$. Hence, we obtain

Proposition $2.2 R_{+}(\cdot)$ has a holomorphic extension to $\mathbb{C}$. The properties (i), (ii) und (iii) of Proposition 2.1 are valid for any $\alpha \in \mathbb{C}$.

For $\alpha \notin\{0,-2,-4,-6, \ldots\} \cup\{n-2, n-4, n-6, \ldots\}$ we have

$$
\operatorname{supp} R_{+}(\alpha)=\mathcal{J}_{+}(0), \quad \operatorname{singsupp} R_{+}(\alpha) \subseteq \mathcal{C}_{+}(0)
$$

and for all other $\alpha$

$$
\operatorname{supp} R_{+}(\alpha)=\operatorname{singsupp} R_{+}(\alpha) \subseteq \mathcal{C}_{+}(0)
$$

holds.

\section{Proposition 2.3}

1. Let be $n \geq 3$ and $k \in\left\{1,2, \ldots,\left[\frac{n-1}{2}\right]\right\}$.

Then we have $\operatorname{supp} R_{+}(n-2 k)=\mathcal{C}_{+}(0)$.

2. $R_{+}(0)=\delta_{0}$, where $\delta_{0}$ is the Dirac measure with support at the origin.

A look at Proposition 2.3 and property (iii) of Proposition 2.1 now shows

Corollary 2.1 $\square R_{+}(2)=R_{+}(0)=\delta_{0}$, i.e. $R_{+}(2)$ is a fundamental solution of $\square$. 


\subsection{Riesz distributions on geodesically normal domains in Lorentzian manifolds}

During this section let $\left(M^{n}, g\right)$ be a Lorentzian manifold and $\mu_{g}$ its volume form.

Definition 2.2 An open subset $\Omega$ of $M^{n}$ is called a geodesically normal domain if it is a normal neighborhood for each of its points. In particular, for any pair $x, y$ of points in $\Omega$ there exists a unique geodesic $c:[0,1] \longmapsto \Omega$ with $c(0)=x, c(1)=y$. Let $\sigma: \Omega \times \Omega \longrightarrow \mathbb{R}$ be the quadratic geodesic distance defined by

$$
\begin{aligned}
& \sigma(x, y):=g_{c(t)}\left(c^{\prime}(t), c^{\prime}(t)\right) \\
& \text { where } c:[0,1] \rightarrow \Omega \text { is the unique geodesic from } x \text { to } y
\end{aligned}
$$

and denote $\sigma_{x}:=\sigma(x, \cdot)$.

Let $\Omega \subset M^{n}$ be a space and time oriented geodesically normal domain. Let $x_{0}$ be in $\Omega$. Then

$$
\begin{aligned}
& \mathcal{J}_{+}^{\Omega}\left(x_{0}\right):= \\
& \quad\left\{x \in \Omega \mid \text { the unique geodesic in } \Omega \text { from } x_{0} \text { to } x \text { is causal and future oriented }\right\}
\end{aligned}
$$

is the future of $x_{0} \in \Omega$ and

$\mathcal{C}_{+}^{\Omega}\left(x_{0}\right):=\partial \mathcal{J}_{+}^{\Omega}\left(x_{0}\right)$

$=\left\{x \in \Omega \mid\right.$ the unique geodesic in $\Omega$ from $x_{0}$ to $x$ is light like and future oriented $\}$

the future light cone of $x_{0} \in \Omega$. Similarly, we define $\mathcal{J}_{-}^{\Omega}\left(x_{0}\right)$ and $\mathcal{C}_{-}^{\Omega}\left(x_{0}\right)$, where now the geodesic is past oriented.

We fix a point $x_{0} \in \Omega$ and a positive oriented pseudo-orthonormal basis $X_{1}, \ldots, X_{n}$ in the tangent space $T_{x_{0}} \Omega$. Here let $X_{1}$ be future oriented. We extend this basis parallel along the geodesics through $x_{0}$ and obtain a local frame $X_{1}(x), \ldots, X_{n}(x)$ on $\Omega$. In this way we get the following trivialization $\pi$ of the tangent space $T \Omega$ :

$$
\begin{aligned}
\pi: \Omega \times \mathbb{R}^{n} & \longrightarrow T \Omega \\
\left(x, \xi_{1}, \ldots, \xi_{n}\right) & \longmapsto \sum_{i=1}^{n} \xi_{i} X_{i}(x) .
\end{aligned}
$$

In particular, $\pi_{x}:=\left.\pi\right|_{\{x\} \times \mathbb{R}^{n}}$ is an isometry from the Minkowski space $\left(\{x\} \times \mathbb{R}^{n}, g_{0}\right)$ to $\left(T_{x} \Omega, g_{x}\right)$.

For any $x \in \Omega$ we now define the map

$$
\begin{aligned}
\mathfrak{a}_{x}: \mathbb{R}^{n} & \stackrel{\pi_{x}}{\longrightarrow} T_{x} \Omega \stackrel{\exp _{x}}{\longrightarrow} \Omega \\
\left(\xi_{1}, \ldots, \xi_{n}\right) & \longmapsto \exp _{x}\left(\sum_{i=1}^{n} \xi_{i} X_{i}(x)\right) .
\end{aligned}
$$


These $\mathfrak{a}_{x}$ are orientation preserving $C^{\infty}$-maps. Their restrictions to $\mathfrak{a}_{x}^{-1}(\Omega)$ are diffeomorphisms from $\mathfrak{a}_{x}^{-1}(\Omega)$ to $\Omega$. The map $\mathfrak{a}_{x}^{-1}$ yields the normal coordinates on $\Omega$ relative to $X_{1}(x), \ldots, X_{n}(x)$.

Furthermore we define by

$$
\left(\mathfrak{a}_{x}\right)^{*} \mu_{g}=m_{x} \cdot \mu_{0}
$$

positive smooth functions $m_{x} \in C^{\infty}\left(\mathfrak{a}_{x}^{-1}(\Omega)\right)$.

Let now $\varphi$ be in $C_{0}^{\infty}(\Omega)$. For any $x \in \Omega$ we have the following function $\widetilde{\varphi}_{x} \in$ $C_{0}^{\infty}\left(\mathbb{R}^{n}\right)$ :

$$
\widetilde{\varphi}_{x}(\xi)=\left\{\begin{array}{cll}
m_{x}(\xi) \cdot \varphi\left(\mathfrak{a}_{x}(\xi)\right) & : \xi \in \mathfrak{a}_{x}^{-1}(\Omega) \\
0 & : & \text { else }
\end{array}\right.
$$

Definition 2.3 Let $\Omega$ be a time and space oriented geodesically normal domain and $x \in \Omega$. The Riesz distribution with respect to $x$ on $\Omega$ is defined by

$$
\left\langle R_{ \pm}^{\Omega}(\alpha, x), \varphi\right\rangle:=\left\langle R_{ \pm}(\alpha), \tilde{\varphi}_{x}\right\rangle \quad, \quad \varphi \in C_{0}^{\infty}(\Omega) .
$$

The properties of the Riesz distributions on the Minkowski space yield the following properties of the Riesz distributions on $\Omega$.

\section{Proposition 2.4}

1. $R_{ \pm}^{\Omega}(\cdot, x) \in \mathcal{D}^{\prime}(\Omega)$ is holomorphic on $\mathbb{C}$.

2. If $\operatorname{Re} \alpha>n$ then

$$
\left\langle R_{ \pm}^{\Omega}(\alpha, x), \varphi\right\rangle=\frac{\pi^{\frac{2-n}{2}} 2^{1-\alpha}}{\Gamma\left(\frac{\alpha}{2}\right) \Gamma\left(\frac{\alpha-n}{2}+1\right)} \int_{\mathcal{J}_{ \pm}^{\Omega}(x)}\left(-\sigma_{x}(y)\right)^{\frac{\alpha-n}{2}} \varphi(y) \mu_{g}(y) .
$$

3. The multiplication by $-\sigma_{x}$ increases the value of $\alpha$ :

$$
-\sigma_{x} \cdot R_{ \pm}^{\Omega}(\alpha, x)=\alpha(\alpha-n+2) R_{ \pm}^{\Omega}(\alpha+2, x)
$$

4. Let $Y \in \mathfrak{X}(\Omega)$ be a smooth vector field on $\Omega$. If we consider $Y$ as a differential operator then

$$
Y\left(-\sigma_{x}\right) \cdot R_{ \pm}^{\Omega}(\alpha, x)=2 \alpha \cdot Y R_{ \pm}^{\Omega}(\alpha+2, x)
$$

holds.

5. For $\alpha \notin\{0,-2,-4,-6, \ldots\} \cup\{n-2, n-4, n-6, \ldots\}$ we have

$$
\operatorname{supp} R_{+}^{\Omega}(\alpha, x)=\mathcal{J}_{+}^{\Omega}(x), \quad \operatorname{singsupp} R_{+}^{\Omega}(\alpha, x) \subseteq \mathcal{C}_{+}^{\Omega}(x)
$$

and for all other $\alpha \in \mathbb{C}$ we get

$$
\operatorname{supp} R_{+}^{\Omega}(\alpha, x)=\operatorname{singsupp} R_{+}^{\Omega}(\alpha, x) \subseteq \mathcal{C}_{+}^{\Omega}(x) .
$$

6. If $n \geq 3$ and $k \in\left\{1,2, \ldots,\left[\frac{n-1}{2}\right]\right\}$ then $\operatorname{supp} R_{+}(n-2 k, x)=\mathcal{C}_{+}^{\Omega}(x)$.

7. $R_{+}^{\Omega}(0, x)=\delta_{x}$ where $\delta_{x}$ is the Dirac distribution with respect to $x$. 


\section{Normally hyperbolic operators}

In this section we define normally hyperbolic operators and explain some of their fundamental analytic properties.

\subsection{Definition and examples}

Definition 3.1 Let $M^{n}$ be a smooth $n$-dimensional manifold, $(E, p, M)$ a real or complex vector bundle over $M$.

A differential operator $P: \Gamma(E) \longrightarrow \Gamma(E)$ of second order on $E$ is called normally hyperbolic if there exists a Lorentzian metric such that the principal symbol $\sigma(P)$ of $P$ is given by $\sigma(P)_{x}(\xi)=-g_{x}(\xi, \xi) I d_{E_{x}}$, where $x \in M$ and $\xi \in T M^{*} \backslash 0$.

We denote by $\mathcal{N H}(E)$ the set of all normally hyperbolic operators on $E$.

Remark In local coordinates on $M$ and a local trivialization of $E$ a normally hyperbolic operator can be expressed in the form

$$
P=-\sum_{i, j=1}^{n} g^{i j}(x) \frac{\partial^{2}}{\partial x_{i} \partial x_{j}}+\sum_{k=1}^{n} A^{k}(x) \frac{\partial}{\partial x_{k}}+B(x)
$$

where $\left(g^{i j}\right)$ is the inverse matrix of the coefficients of the Lorentzian metric.

\section{Examples}

1. The wave operator of a Riemannian manifold

Let $(X, h)$ be a Riemannian manifold and let $\Delta_{X}$ be the Laplace-Beltrami operator of $(X, h)$. Consider the Lorentzian product $M:=\mathbb{R} \times X ; g:=-d t^{2}+h$. Then the wave operator

$$
\square_{X}:=-\frac{\partial^{2}}{\partial t^{2}}+\Delta_{X}: C^{\infty}(M) \rightarrow C^{\infty}(M)
$$

is normally hyperbolic.

2. The Yamabe operator of a Lorentzian manifold

Let $\left(M^{n}, g\right)$ be a Lorentzian manifold with scalar curvature $R$ and let $\Delta_{0}$ denote the Laplace-Beltrami operator of $\left(M^{n}, g\right)$. Then the Yamabe operator

$$
Y=\Delta_{0}+\frac{n-2}{4(n-1)} R: C^{\infty}(M) \rightarrow C^{\infty}(M)
$$

is normally hyperbolic.

3. The Hodge-Laplace operator on forms of a Lorentzian manifold

Let $\left(M^{n}, g\right)$ be an oriented Lorentzian manifold. Then the Hodge-Laplace operator on $\mathrm{k}$-forms

$$
\Delta_{k}=d d^{*}+d^{*} d: \Omega^{k}(M) \longrightarrow \Omega^{k}(M)
$$

is normally hyperbolic. 
4. The Dirac operator of a Lorentzian spin manifold

Let $(M, g)$ be an oriented Lorentzian spin manifold with spinor bundle $S$. Then the square of the Dirac operator $D$

$$
D^{2}: \Gamma(S) \longrightarrow \Gamma(S)
$$

is normally hyperbolic. The same is true for coupled Dirac operators.

\subsection{The Weitzenböck formula}

To each normally hyperbolic operator a covariant derivative and a homomorphism can be associated which we will define now.

Let $\nabla$ be a covariant derivative on a vector bundle $E$ over a Lorentzian manifold $(M, g)$. Let denote by $\nabla^{T^{*} M \otimes E}$ the covariant derivative defined by the Levi-Civita connection of $(M, g)$ and $\nabla$. The operator

$$
\Delta^{\nabla}:=-\operatorname{Trace}_{g}\left(\nabla^{T^{*} M \otimes E} \circ \nabla\right)
$$

is called Bochner Laplace operator defined by $\nabla$.

A normally hyperbolic operator has the following property

Proposition 3.1 Let $P: \Gamma(E) \longrightarrow \Gamma(E)$ be a normally hyperbolic operator on $E$ and let us denote by $g_{P}$ the Lorentzian metric given by the principal symbol of $P$. Then there exist a uniquely determined covariant derivative $\nabla^{P}: \Gamma(E) \longrightarrow \Gamma\left(T^{*} M \otimes E\right)$ and a homomorphism $H_{P} \in \Gamma(\operatorname{Hom}(E, E))$ such that

$$
P=\Delta^{\nabla^{P}}+H_{P}
$$

Notation In the following we will write $\Delta^{P}$ instead of $\Delta^{\nabla^{P}}$.

Proof. For an arbitrary covariant derivative $\nabla$, a function $f \in C^{\infty}(\mathrm{M})$, and a section $s \in \Gamma(E)$ we have

$$
\left[\Delta^{\nabla}, f\right] s:=\Delta^{\nabla}(f s)-f \Delta^{\nabla} s=-2 \nabla_{\operatorname{grad}(f)} s+\left(\Delta_{0} f\right) s
$$

Hence, if $\nabla$ is a covariant derivative such that $P-\Delta^{\nabla}$ is a homomorphism, then $\nabla$ has to satisfy the condition

$$
\nabla_{h \operatorname{grad}(f)} s=\frac{1}{2} h\left\{\left(\Delta_{0} f\right) s-P(f s)+f P(s)\right\}
$$

But, (4) defines a covariant derivative on $E$ for which (3) is satisfied.

(3) is called Weitzenböck formula for $P$. Hence, a Lorentzian metric on $M$, a covariant derivative and a homomorphism on $E$ are associated to each normally hyperbolic operator $P \in \mathcal{N H}(E)$. 


\section{Examples}

1. Yamabe operator

For the Yamabe operator $Y$ the covariant derivative is given by $\nabla_{X} f=X(f)$ and the homomorphism is $H=\frac{n-2}{4(n-1)} R$.

2. Hodge-Laplace operator on forms

For the Hodge-Laplace operator on $k$-forms the covariant derivative is given by the Levi-Civita connection of the Lorentzian manifold $(M, g)$ and the homomorphism can be expressed by the curvature of $g$. For example, for 1 -forms the homomorphism $H$ is the Ricci curvature of $g$ which is considered as homomorphism on $T^{*} M$

\section{Dirac operator}

For the square of the classical Dirac operator $D^{2}: \Gamma(S) \longrightarrow \Gamma(S)$ on a Lorentzian spin manifold the covariant derivative is the usual spinor derivative $\nabla^{S}$ and the homomorphism is $\frac{1}{4} R$.

Let $A$ be a connection in a principal fibre bundle $Q$ and let $F^{A}$ denote the curvature defined by $A$ in a vector bundle $E$ associated to $Q$. Then, for the square of the Dirac operator $D_{A}^{2}: \Gamma(S \otimes E) \longrightarrow \Gamma(S \otimes E)$ coupled to the connection $A$, the covariant derivative and the homomorphism corresponding to $D_{A}^{2}$ are given by

$$
\begin{aligned}
\nabla & =\nabla^{S} \otimes 1+1 \otimes \nabla^{A} \\
H & =\frac{1}{4} R+\sum_{i<j} s_{i} \cdot s_{j} \cdot \otimes F^{A}\left(s_{i}, s_{j}\right)
\end{aligned}
$$

where $\left(s_{1}, \ldots, s_{n}\right)$ denotes a local orthonormal basis on $(M, g)$ and $X \cdot$ is the Clifford multiplication by the vector field $X$.

\subsection{Hadamard coefficients}

For two vector bundles $E$ and $F$ over $M^{n}$ we denote by $E \otimes F$ the external tensor product of these bundles over $M \times M$ :

$$
E \otimes F:=p r_{1}^{*} E \otimes p r_{2}^{*} F .
$$

We often identify the fibre of $E^{*} \otimes E$ over a point $(x, y) \in M \times M$ with the set of homomorphisms $\operatorname{Hom}\left(E_{x}, E_{y}\right)$. Let $\Omega \subset M$ be a geodesically normal domain in $M$. To each normally hyperbolic operator $P$ we can associate a sequence of smooth sections $U_{k} \in \Gamma\left(\Omega \times \Omega, E^{*} \otimes E\right), k=0,1,2, \ldots$, the so-called Hadamard coefficients of $P$, which we will define in the following.

Let $\mathbf{r}_{x}$ denote the radial vector field on $\Omega$ with respect to $x \in \Omega$ and recall that $\sigma: \Omega \times \Omega \longrightarrow \mathbb{R}$ is the quadratic geodesic distance function on $\Omega$ and $\sigma_{x}: \Omega \longrightarrow \mathbb{R}$ is 
given by $\sigma_{x}(y):=\sigma(x, y)$. Then

$$
\begin{aligned}
\mathbf{r}_{x} & =\frac{1}{2} \operatorname{grad} \sigma_{x} \\
\mathbf{r}_{x}(\gamma(t)) & =t \gamma^{\prime}(t)
\end{aligned}
$$

where $\gamma$ is a geodesic starting at $x$. The function $m \in C^{\infty}(\Omega \times \Omega)$

$$
m(x, \cdot):=-\frac{1}{2} \Delta \sigma_{x}-n
$$

is called divergence measure of $\Omega$. By $\tau \in C^{\infty}(\Omega \times \Omega)$ we denote the function

$$
\tau(x, y):=\exp \left\{\frac{1}{2} \int_{0}^{1} \frac{m(x, \gamma(s))}{s} d s\right\}
$$

where $\gamma:[0,1] \longrightarrow \Omega$ denotes the unique geodesic in $\Omega$ joining $x$ and $y$. The function $\tau_{x}: \Omega \longrightarrow \mathbb{R}$ is given by $\tau_{x}(y):=\tau(x, y)$. Then it is easy to check that

$$
\mathbf{r}_{x}\left(\tau_{x}\right)=\frac{1}{2} \tau(x, \cdot) m(x, \cdot)
$$

Proposition 3.2 Let $P \in \mathcal{N H}(E)$ be a normally hyperbolic operator and let $\Omega \subset M$ be a geodesically normal domain. Then there exists a uniquely determined sequence of sections $U_{k} \in \Gamma\left(\Omega \times \Omega, E^{*} \otimes E\right), k=0,1,2, \ldots$, such that the following differential equations and initial conditions are satisfied on $\Omega$ for all $x \in \Omega$ :

$$
\begin{gathered}
\nabla_{\mathbf{r}_{x}}^{P} U_{k}(x, \cdot)+\left(\frac{1}{2} m(x, \cdot)+k\right) U_{k}(x, \cdot)=-\frac{1}{2} P\left(U_{k-1}(x, \cdot)\right) \\
U_{-1}=0 \\
U_{0}(x, x)=I d_{E_{x}}
\end{gathered}
$$

Let denote $\mathcal{P}(x, y) \in \operatorname{Hom}\left(E_{x}, E_{y}\right)$ the parallel displacement along the geodesic $\gamma$, joining $x$ and $y$ in $\Omega$. Then the sections $U_{k}$ satisfy

$$
\begin{aligned}
U_{0}(x, y) & =\frac{1}{\tau(x, y)} \mathcal{P}(x, y) \\
U_{k}(x, y) & =-\frac{1}{2 \tau(x, y)} \int_{0}^{1} t^{1-k} \tau(x, \gamma(t)) \mathcal{P}(\gamma(t), y) P\left(U_{k-1}(x, \cdot)\right)(\gamma(t)) d t
\end{aligned}
$$

All differentiations refer to the second component.

Proof. Consider first the case $k=0$ : Let $U_{0}$ be a solution of (8) for $k=0$, considered as a homomorphism. Using (7), we obtain for each $e \in E_{x}$

$$
\begin{aligned}
\frac{1}{\tau(x, \cdot)} \nabla_{\mathbf{r}_{x}}\left[\tau_{x} U_{0}(x, \cdot)(e)\right] & =\frac{1}{\tau(x, \cdot)} \mathbf{r}_{x}\left(\tau_{x}\right) U_{0}(x, \cdot) e+\nabla_{\mathbf{r}_{x}}\left(U_{0}(x, \cdot) e\right) \\
& =\frac{1}{2} m(x, \cdot) U_{0}(x, \cdot) e+\nabla_{\mathbf{r}_{x}}\left(U_{0}(x, \cdot) e\right) \\
& =0
\end{aligned}
$$


Hence $\tau(x, \cdot) U_{0}(x, \cdot) e$ is parallel along each geodesic starting at $x$. From the initial condition for $U_{0}$ results

$$
\tau(x, x) U_{0}(x, x) e=e
$$

Therefore, the homomorphism $\tau(x, y) U_{0}(x, y)$ is the parallel displacement from $E_{x}$ to $E_{y}$ along the geodesic $\gamma$, joining $x$ and $y$. On the other hand it is easy to see by differentiation, that $U_{0}(x, y)=\frac{1}{\tau(x, y)} \mathcal{P}(x, y)$ is a solution of $(8)$ for $k=0$.

Now, let $U_{k}$ be a solution of (8) for $k>0$. Using (6) and (7) we obtain

$$
\begin{aligned}
& \left\{\nabla_{\mathbf{r}_{x}}\left[t^{k} \tau_{x} U_{k}(x, \cdot) e\right]\right\}(\gamma(t)) \\
& =\mathbf{r}_{x}\left[t^{k} \tau_{x}\right]\left(U_{k}(x, \cdot) e\right) \gamma(t)+t^{k} \tau(x, \gamma(t))\left\{\nabla_{\mathbf{r}_{x}}\left(U_{k}(x, \cdot) e\right\}(\gamma(t))\right. \\
& =\left\{k t^{k} \tau(x, \gamma(t))+\frac{1}{2} t^{k} \tau(x, \gamma(t)) m(x, \gamma(t))\right\} U_{k}(x, \gamma(t)) e+ \\
& \quad t^{k} \tau(x, \gamma(t))\left\{\nabla_{\mathbf{r}_{x}}\left(U_{k}(x, \cdot) e\right)\right\}(\gamma(t)) \\
& =t^{k} \tau(x, \gamma(t))\left\{\left[k+\frac{1}{2} m(x, \gamma(t))\right] U_{k}(x, \gamma(t)) e+\left[\nabla_{\mathbf{r}_{x}}\left(U_{k}(x, \cdot) e\right)\right](\gamma(t))\right\} \\
& =-\frac{1}{2} t^{k} \tau(x, \gamma(t))\left[P\left(U_{k-1}(x, \cdot) e\right)\right](\gamma(t)) .
\end{aligned}
$$

Hence, using the relation of the covariant derivative $\nabla$ and the parallel displacement $\mathcal{P}$

$$
\nabla_{\gamma^{\prime}(t)} \hat{e}=\left.\frac{d}{d s}[\mathcal{P}(\gamma(s), \gamma(t)) \hat{e}(\gamma(s))]\right|_{s=t} \quad \text { for } \quad \hat{e} \in \Gamma(E)
$$

we obtain

$$
\begin{gathered}
\left.\frac{d}{d s}\left\{\mathcal{P}(\gamma(s), \gamma(t))\left[s^{k} \tau(x, \gamma(s)) U_{k}(x, \gamma(s)) e\right]\right\}\right|_{s=t} \\
=-\frac{1}{2} t^{k-1} \tau(x, \gamma(t))\left[P\left(U_{k-1}(x, \cdot) e\right)\right](\gamma(t))
\end{gathered}
$$

By applying $\mathcal{P}(\gamma(t), y)$ to both sides of (11) and integrating, the following results

$$
\tau(x, y) U_{k}(x, y) e=-\frac{1}{2} \int_{0}^{1} t^{k-1} \tau(x, \gamma(t)) \mathcal{P}(\gamma(t), y)\left[P\left(U_{k-1}(x, \cdot) e\right)\right](\gamma(t)) d t
$$

Hence $U_{k}$ satisfies (10). On the other hand it is easy to check by differentiation that a section $U_{k}$ defined by (10) satisfies (8).

Definition 3.2 The sections $U_{k} \in \Gamma\left(\Omega \times \Omega, E^{*} \otimes E\right)$ described in Proposition 3.2 are called Hadamard coefficients of $P$ with respect to $\Omega$.

\subsection{Fundamental solution}

Let $(E, p, M)$ be a vector bundle over $\mathrm{M}, \Omega \subset M$ a domain , $x \in \Omega$ and $V$ a vector space. By $\mathcal{D}^{\prime}\left(\Omega, E^{*} ; V\right)$ we denote the space of distributions on $\left.E^{*}\right|_{\Omega}$ with values in $V$

$$
\mathcal{D}^{\prime}\left(\Omega, E^{*} ; V\right):=\left\{T: \Gamma_{0}\left(\Omega, E^{*}\right) \longrightarrow V \mid T \text { linear and continuous }\right\}
$$


Each differential operator $P$ on $E$ extends to $\mathcal{D}^{\prime}\left(\Omega, E^{*} ; V\right)$ in the following way. If $P^{*}$ denotes the dual operator on $E^{*}$, defined by

$$
\int_{M} \psi(P \varphi) \mu=\int_{M}\left(P^{*} \psi\right)(\varphi) \mu, \quad \psi \in \Gamma_{0}\left(E^{*}\right), \varphi \in \Gamma_{0}(E)
$$

then for each distribution $T \in \mathcal{D}^{\prime}\left(\Omega, E^{*} ; V\right)$ the distribution $P T$ is defined by

$$
\langle P T, u\rangle:=\left\langle T, P^{*} u\right\rangle \quad u \in \Gamma_{0}\left(\Omega, E^{*}\right)
$$

A distribution $G \in \mathcal{D}^{\prime}\left(\Omega, E^{*} ; E_{x}^{*}\right)$ is called fundamental solution of $P$ with respect to $(\Omega, x)$, if $P G=\delta_{x}^{E^{*}}$, where $\delta_{x}^{E^{*}}$ is the Dirac distribution of $E_{\Omega}^{*}$ supported in $x$ : $\delta_{x}^{E^{*}}(u):=u(x)$.

In general there exists no fundamental solution of a normally hyperbolic operator $P$ with respect to $(\Omega, x)$. One has to restrict oneself to a certain class of domains $\Omega$, the so-called causal domains.

Definition 3.3 A domain $\Omega_{0} \subset M$ is called causal domain if

1. $\Omega_{0}$ is contained in a time and space oriented geodesically normal domain $\Omega$ and

2. $\mathcal{J}_{+}^{\Omega}(x) \cap \mathcal{J}_{-}^{\Omega}(y)$ is compact (or empty) and contained in $\Omega_{0}$ for all $x, y \in \Omega_{0}$.

Proposition 3.3 ([Fri75], Th.4.4.1)

Each Lorentzian manifold can be covered by causal domains.

Definition 3.4 A subset $A \subset \Omega_{0}$ of a causal domain $\Omega_{0}$ is called past compact (or future compact), if $A \cap \mathcal{J}_{-}^{\Omega_{0}}(x)$ (or $A \cap \mathcal{J}_{+}^{\Omega_{0}}(x)$ ) is compact (or empty) for all $x \in \Omega_{0}$.

Notation: $\mathcal{D}_{+}^{\prime}\left(\Omega_{0}, E^{*} ; E_{x}^{*}\right) \subset \mathcal{D}^{\prime}\left(\Omega_{0}, E^{*} ; E_{x}^{*}\right)$ denotes the subset of all distributions with past compact support. $\mathcal{D}_{-}^{\prime}\left(\Omega_{0}, E^{*} ; E_{x}^{*}\right) \subset \mathcal{D}^{\prime}\left(\Omega_{0}, E^{*} ; E_{x}^{*}\right)$ denotes the subset of all distributions with future compact support.

Theorem 3.1 ([Gün88], chap.3.3)

Let $P \in \mathcal{N} \mathcal{H}(E)$ be a normally hyperbolic operator, let $\Omega_{0} \subset M$ be a causal domain and $x \in \Omega_{0}$. Then there exists exactly one fundamental solution $G_{+}^{\Omega_{0}}(x) \in \mathcal{D}_{+}^{\prime}\left(\Omega_{0}, E^{*} ; E_{x}^{*}\right)$ of $P$ with respect to $\left(\Omega_{0}, x\right)$ with past compact support (forward fundamental solution) and there exists exactly one fundamental solution $G_{-}^{\Omega_{0}}(x) \in \mathcal{D}_{-}^{\prime}\left(\Omega_{0}, E^{*} ; E_{x}^{*}\right)$ of $P$ with respect to $\left(\Omega_{0}, x\right)$ with future compact support (backward fundamental solution).

The support and singular support of these fundamental solutions satisfy

$$
\begin{aligned}
\operatorname{supp} G_{ \pm}^{\Omega_{0}}(x) & \subset \mathcal{J}_{ \pm}^{\Omega_{0}}(x) \\
\operatorname{singsupp} G_{ \pm}^{\Omega_{0}}(x) & \subset \mathcal{C}_{ \pm}^{\Omega_{0}}(x)
\end{aligned}
$$

If $n$ is odd or $n=2$, then the fundamental solution has the following structure:

$$
G_{ \pm}^{\Omega_{0}}(x)=T(x, .) R_{ \pm}^{\Omega_{0}}(2, x)
$$

where $R_{ \pm}^{\Omega_{0}}(2, x) \in \mathcal{D}^{\prime}\left(\Omega_{0}\right)$ is the Riesz distribution of the Lorentzian manifold for the parameter $\alpha=2$ and $T \in \Gamma\left(\Omega_{0} \times \Omega_{0}, E^{*} \otimes E\right)$ is a smooth section with the following 
asymptotic behaviour at the zero set $C=\left\{(x, y) \in \Omega_{0} \times \Omega_{0} \mid \sigma(x, y)=0\right\}$ of the quadratic geodesic distance function $\sigma$ :

$$
T(x, y) \underset{(x, y) \rightarrow C}{\sim} \sum_{k=0}^{\infty}(-1)^{k} c_{(4-n, k)}^{-1} U_{k}(x, y) \sigma^{k}(x, y)
$$

If $n$ is even and $n \geq 4$, then the fundamental solution has the following structure

$$
G_{ \pm}^{\Omega_{0}}(x)=\sum_{k=0}^{\frac{n-4}{2}} c_{(2, k)} U_{k}(x, .) R_{ \pm}^{\Omega_{0}}(2+2 k, x)+c_{\left(2, \frac{n-2}{2}\right)} T(x, .) R_{ \pm}^{\Omega_{0}}(n, x)
$$

where $R_{ \pm}^{\Omega_{0}}(\alpha, x)$ are the Riesz distributions of the Lorentzian manifold and $T \in \Gamma\left(\Omega_{0} \times\right.$ $\left.\Omega_{0}, E^{*} \otimes E\right)$ is a smooth section with the following asymptotic behaviour at the set $C$ :

$$
T(x, y) \underset{(x, y) \rightarrow C}{\underset{1}{2}} \sum_{k=0}^{\infty}(-1)^{k} c_{(2, k)}^{-1} U_{\frac{n-2}{2}+k}(x, y) \sigma^{k}(x, y)
$$

Here, for $a \in \mathbb{C}$ and $k \in \mathbb{N}, c_{(a, k)}$ denotes the number

$$
c_{(a, 0)}:=1, \quad c_{(a, k)}:=a(a+2)(a+4) \cdots(a+2 k-2)
$$

Definition 3.5 The smooth section $T \in \Gamma\left(\Omega_{0} \times \Omega_{0}, E^{*} \otimes E\right)$ appearing in the regular part of the fundamental solution $G_{ \pm}^{\Omega_{0}}(x)$ for even dimension $n \geq 4$ (see (12)) is called tail term of the fundamental solution $G_{ \pm}^{\Omega_{0}}(x)$.

\section{Huygens operators}

Let $(M, g)$ be a Lorentzian manifold. We consider a real or complex vector bundle $\mathrm{E}$ over $\mathrm{M}$ and a normally hyperbolic operator $P: \Gamma(E) \longrightarrow \Gamma(E)$.

Definition 4.1 Let $\Omega_{0} \subset M$ be a causal domain and $x \in \Omega_{0}$. The operator $P$ is called a Huygens operator with respect to $\left(\Omega_{0}, x\right)$ if its forward and its backward fundamental solution $G_{ \pm}^{\Omega_{0}}(x)$ satisfy $\operatorname{supp} G_{ \pm}^{\Omega_{0}}(x) \subset \mathcal{C}_{ \pm}^{\Omega_{0}}(x)$.

$P$ is called a Huygens operator or an operator of Huygens type if there exists a covering $\Sigma$ of $M$ by causal domains such that $P$ is a Huygens operator with respect to all $\left(\Omega_{0}, x\right)$ with $\Omega_{0} \in \Sigma, x \in \Omega_{0}$.

We denote the set of all Huygens operators on $E$ by $\mathcal{H U \mathcal { Y }}(E)$.

Huygens operators can be characterized also in the following way.

Theorem 4.1 [Gün88] Let $\Omega_{0} \subset M$ be a causal domain and $x \in \Omega_{0}$. The operator $P$ is a Huygens operator with respect to $\left(\Omega_{0}, x\right)$ if for any distribution with compact support $f \in \mathcal{E}^{\prime}\left(\Omega_{0}, E^{*}\right)$ and supp $f \cap \mathcal{C}_{-}^{\Omega_{0}}(x)=\emptyset$ the forward solution $u_{+}$of $P u_{+}=f$ and for any $g \in \mathcal{E}^{\prime}\left(\Omega_{0}, E^{*}\right)$ with $\operatorname{supp} g \cap \mathcal{C}_{+}^{\Omega_{0}}(x)=\emptyset$ the backward solution $u_{-}$of $P u_{-}=g$ vanish at $x$. 
Another characterization of Huygens operators can be given by properties of solutions of the Cauchy problem (see [Gün88] Th. 4.1.12).

Using the description of the fundamental solutions $G_{ \pm}^{\Omega_{0}}(x)$ given in Theorem 3.1 and the properties of $\operatorname{supp} R_{ \pm}^{\Omega_{0}}(x, \alpha)$ one obtains

Theorem 4.2 1. If the dimension $n$ of $M$ is odd or equal to 2 , then $P$ can never be a Huygens operator.

2. If $n$ is even and $n \geq 4$ then $P$ is a Huygens operator if and only if there exists a covering $\Sigma$ by causal domains such that the tail term $T(x, y)$ of the fundamental solutions $G_{+}^{\Omega_{0}}(x)$ and $G_{-}^{\Omega_{0}}(x)$ with respect to $\left(\Omega_{0}, x\right)$ satisfies

$$
T(x, y)=0
$$

for all $\Omega_{0} \in \Sigma, x \in \Omega_{0}, y \in \mathcal{J}^{\Omega_{0}}(x)$.

Using the asymptotic expansion (13) of $T(x, y)$ one obtains

Theorem 4.3 (Hadamard Criterion) If $n$ is even and $n \geq 4$ then $P$ is a Huygens operator if and only if there exists a covering $\Sigma$ by causal domains such that the Hadamard coefficient $U_{\frac{n-2}{2}}$ of $P$ satisfies

$$
U_{\frac{n-2}{2}}(x, y)=0
$$

for all $\Omega_{0} \in \Sigma, x \in \Omega_{0}, y \in \mathcal{C}^{\Omega_{0}}(x)$

or, equivalently, that the Hadamard coefficient $U_{\frac{n-4}{2}}$ of $P$ satisfies

$$
P\left(U_{\frac{n-4}{2}}(x, \cdot)\right)(y)=0
$$

for all $\Omega_{0} \in \Sigma, x \in \Omega_{0}, y \in \mathcal{C}^{\Omega_{0}}(x)$.

Example Let $\left(\mathbb{R}^{n, 1}, g_{0}\right)$ be the Minkowski space, $n \geq 4$ and $n$ even. Consider the Laplace-Beltrami operator

$$
\Delta_{0}=\frac{\partial^{2}}{\partial x_{1}^{2}}-\frac{\partial^{2}}{\partial x_{2}^{2}}-\ldots-\frac{\partial^{2}}{\partial x_{n}^{2}}
$$

of $\left(\mathbb{R}^{n, 1}, g_{0}\right)$. Using (9) it can be checked that the Hadamard coefficient $U_{0}$ of $\Delta_{0}$ is given by $U_{0} \equiv 1$. Hence, $U_{k} \equiv 0$ for all $k \geq 1$. Therefore, $\Delta_{0}$ is a Huygens operator.

\section{Conformal gauge invariance of Huygens operators}

Let $M^{n}$ be a n-dimensional manifold, $E$ a real or complex vector bundle over $M$ and $P: \Gamma(E) \longrightarrow \Gamma(E)$ a normally hyperbolic operator with principal symbol given by a metric $g$. Furthermore let $\varphi \in C^{\infty}(M)$ be a smooth function and $A \in \Gamma($ Aut $E)$ an invertible homomorphism. 
Definition 5.1 1. The operator $P_{\varphi, A}: \Gamma(E) \longrightarrow \Gamma(E)$ defined by

$$
P_{\varphi, A}(u)=e^{-\frac{n+2}{2} \varphi} \cdot A^{-1} P\left(e^{\frac{n-2}{2} \varphi} \cdot A u\right)
$$

is called the conformal gauge transform of $P$.

2. Let $\widetilde{P}: \Gamma(E) \longrightarrow \Gamma(E)$ be a further second order differential operator. The operators $P, \widetilde{P}$ are called conformal gauge equivalent if there exist a smooth function $\varphi \in C^{\infty}(M)$ and an invertible homomorphism $A \in \Gamma($ Aut $E)$ such that $\widetilde{P}=P_{\varphi, A}$.

A staightforward calculation leads to

Proposition 5.1 Let $\widetilde{P}=P_{\varphi, A}$ be a conformal gauge transform of the normally hyperbolic operator $P$. Then $\widetilde{P}$ is normally hyperbolic. The principal symbol of $\widetilde{P}$ is given by the metric $\widetilde{g}=e^{2 \varphi} g$. The covariant derivative $\nabla^{\widetilde{P}}$ and the homomorphism $H_{\widetilde{P}}$ in $E$ arising from the Weitzenböck formula for $\widetilde{P}$ relates to those of $P$ denoted by $\nabla^{P}$ and $H_{P}$ in the following way

$$
\begin{gathered}
\nabla^{\widetilde{P}}=A^{-1} \nabla^{P} A \\
H_{\widetilde{P}}=e^{-2 \varphi}\left(A^{-1} H_{P} A+e^{-\frac{n-2}{2} \varphi} \Delta_{g}\left(e^{\frac{n-2}{2} \varphi}\right)\right)
\end{gathered}
$$

Here $\Delta_{g}$ denotes the Laplace-Beltrami operator with respect to $g$.

Consequently, the group $\mathcal{G}_{c}(E):=C^{\infty}(M) \times \Gamma(\operatorname{Aut} E)$ acts from the right on the set of normally hyperbolic operators of $E$. It is called the conformal gauge group.

Proposition 5.2 ([Gün88] Th. 6.5.1)

Let $\widetilde{P}=P_{\varphi, A}$ be the conformal gauge transform of a normally hyperbolic operator $P$ on a bundle $E$ over a manifold of even dimension $n \geq 4$. Let $T, \widetilde{T} \in \Gamma\left(\Omega_{0} \times \Omega_{0}, E^{*} \otimes E\right)$ denote the tail terms of the fundamental solution of $P$ and $\widetilde{P}$, respectively. Considered as elements in $\operatorname{Hom}\left(E_{x}, E_{y}\right)$ the following transformation law holds:

$$
\widetilde{T}(x, y)=e^{-\frac{n-2}{2}(\varphi(x)+\varphi(y))} A^{-1}(y) T(x, y) A(x) \quad x \in \Omega_{0}, y \in \mathcal{J}_{ \pm}^{\Omega_{0}}(x)
$$

As a corollary we obtain that the subset of Huygens operators in the set of all normally hyperbolic operators on $E$ is invariant under the action of the conformal gauge group:

Theorem 5.1 (Conformal gauge invariance of $\mathcal{H U} \mathcal{Y}(E)$ )

Let $n \geq 4$ be even. Then $\mathcal{H U} \mathcal{Y}(E) \cdot \mathcal{G}_{c}(E) \subset \mathcal{H U} \mathcal{Y}(E)$.

Definition 5.2 A normally hyperbolic operator $P$ acting on a $\mathbb{K}(=\mathbb{R}, \mathbb{C})$-vector bundle $E$ of rank $m$ is called trivial if for each $x \in M^{n}$ there exists a coordinate neighbourhood $U$ and a trivialization of $\left.E\right|_{U}$ such that the operator $\left.P\right|_{U}$ acting on $\Gamma\left(\left.E\right|_{U}\right) \simeq C^{\infty}\left(U, \mathbb{K}^{m}\right)$ is conformally gauge equivalent to $m$ copies $\left(\triangle_{0}, \ldots, \triangle_{0}\right)$ of the Laplace-Beltrami operator of the Minkowski space. 
Since the Laplacian of the Minkowski space of even dimension $n \geq 4$ is Huygens, the conformal gauge invariance of the set of Huygens operators yields that each trivial operator on a manifold of even dimension $n \geq 4$ is of Huygens type.

Let $P$ be a normally hyperbolic operator on a bundle $E$. Denote by $W_{P} \in \Gamma\left(\left(T^{*} M\right)^{4}\right)$ the Weyl tensor of the Lorentzian metric $g_{P}$ associate to $P$ and by $F^{P} \in \Gamma\left(\Lambda^{2} M \otimes\right.$ $\operatorname{Hom}(E, E))$ the bundle curvature of the covariant derivative $\nabla^{P}$ associated to $P$. Furthermore, let

$$
C_{P}:=H_{P}-\frac{n-2}{4(n-1)} R \in \Gamma(\operatorname{Hom}(E, E))
$$

be the so called Cotton invariant of $P$, where $H_{P}$ denotes the homomorhism in the Weitzenböck formula of $P$ and $R$ is the scalar curvature of $g_{P}$.

Then we have the following criterion for the triviality of a normally hyperbolic operator:

Theorem 5.2 ([Sch78], Prop.2.8)

A normally hyperbolic operator $P \in \mathcal{N H}(E)$ is trivial if and only if $W_{P}=0, F^{P}=0$ and $C_{P}=0$.

This theorem provides further examples of Huygens operators:

1. The Yamabe operator $Y=\Delta_{0}+\frac{n-2}{4(n-1)} R$ on a conformally flat Lorentzian manifold is trivial. In particular, the operator $\Delta_{0}+\frac{n(n-2)}{4} K$ on a Lorentzian manifold $\left(M^{n}, g\right)$ of constant sectional curvature $K$ and the shifted wave operator $P=\frac{\partial^{2}}{\partial t^{2}}+\Delta_{F}+\frac{(n-2)^{2}}{4} K_{0}$ on a Lorentzian product of $\mathbb{R}$ with a Riemannian manifold $\left(F^{n-1}, h\right)$ of constant sectional curvature $K_{0}$ are trivial.

2. Let $\left(M^{n}, g\right)$ be a Lorentzian spin manifold with spinor bundle $S$ and spinor derivative $\nabla^{S}$. A spinor field $\varphi \in \Gamma(S)$ is called a Killing spinor to the Killing number $\lambda \in \mathbb{C}$ if $\nabla_{X}^{\lambda} \varphi:=\nabla_{X}^{S} \varphi-\lambda X \cdot \varphi=0$ for all vector fields $X$ on $M$.

Now, let $\left(M^{n}, g\right)$ be a simply connected Lorentzian manifold of constant sectional curvature $K$. Then the spinor bundle $S$ of $\left(M^{n}, g\right)$ can be trivialized by Killing spinors to the Killing number $\mu$, where $\mu^{2}=\frac{1}{4} K$. Denote by $D$ the Dirac operator of $\left(M^{n}, g\right)$. The Weitzenböck formula for the operator $(D-\mu)^{2}$ is

$$
(D-\mu)^{2}=\Delta^{\nabla^{\mu}}+\frac{1}{4}(n-1)^{2} K
$$

Hence the operator $(D-\mu)^{2}-\frac{1}{4} K$ is trivial.

In chapter 7 we give examples of non-trivial Huygens operators.

To determine the class of all Huygens operators on a given bundle, it is necessary to find a system of conformal gauge invariants which determine the subset $\mathcal{H U} \mathcal{Y}(E)$ of Huygens operators in the set $\mathcal{N H}(E)$ of all normally hyperbolic operators. We now define the notion of a conformal gauge invariant. In the next section we describe the construction of the so-called moments of a normally hyperbolic operator introduced by Günther in [Gün88], chap.6, which serve as a complete system of conformal gauge invariants determing $\mathcal{H U Y}(E)$. 
Definition 5.3 Denote by $\mathcal{E}_{r s t u}$ the bundle $\otimes^{r} T^{*} M \otimes \otimes^{s} T M \otimes \otimes^{t} E^{*} \otimes \otimes^{u} E$ over $M$.

An invertible homomorphism $A \in \Gamma(\operatorname{Aut} E)$ acts on $\mathcal{E}_{r s t u}$ in the following way. First the action of $A$ on $E^{*}$ is defined by $A f=\left(A^{*}\right)^{-1}(f)$ for any $f \in E^{*}$. Then we can define the action on $\mathcal{E}_{r s t u}$ by

$$
\begin{aligned}
& A \cdot\left(\tau_{1} \otimes \ldots \otimes \tau_{r} \otimes t_{1} \otimes \ldots \otimes t_{s} \otimes f_{1} \otimes \ldots \otimes f_{k} \otimes e_{1} \otimes \ldots \otimes e_{l}\right)= \\
& \quad=\tau_{1} \otimes \ldots \otimes \tau_{r} \otimes t_{1} \otimes \ldots \otimes t_{s} \otimes A f_{1} \otimes \ldots \otimes A f_{k} \otimes A e_{1} \otimes \ldots \otimes A e_{l}
\end{aligned}
$$

for all $\tau_{1} \otimes \ldots \otimes \tau_{r} \otimes t_{1} \otimes \ldots \otimes t_{s} \otimes f_{1} \otimes \ldots \otimes f_{k} \otimes e_{1} \otimes \ldots \otimes e_{l} \in \mathcal{E}_{r s t u}$

Definition 5.4 Let $\mathcal{P}$ be a set of normally hyperbolic operators closed under the action of the conformal gauge group $\mathcal{G}_{c}(E)$. A map $T: \mathcal{P} \longmapsto \Gamma\left(\mathcal{E}_{r s t u}\right)$ is called a conformal gauge invariant of weight $\omega$ if

$$
T\left(P_{\varphi, A}\right)=e^{2 \omega \varphi} A^{-1} \cdot T(P)
$$

for all $(\varphi, A) \in \mathcal{G}_{c}(E)$ and all $P \in \mathcal{P}$.

Examples of conformal gauge invariants are

1. the Cotton invariant $C: \mathcal{P} \ni P \longrightarrow C_{P} \in \Gamma\left(E^{*} \otimes E\right)=\Gamma(\operatorname{Hom}(E, E))$ which assigns to an operator $P$ the Cotton invariant of the Lorentzian metric $g_{P}$ associated to $P$. Its weight equals $\omega=-1$.

2. the bundle curvature $F: \mathcal{P} \ni P \longrightarrow F^{P} \in \Gamma\left(\Lambda^{2} M \otimes \operatorname{Hom}(E, E)\right)$ which assigns to an operator $P$ the curvature of that covariant derivative $\nabla^{P}$ in $E$ that occurs in the Weitzenböck formula for $P$. The weight of $F$ equals $\omega=0$.

3. the Weyl tensor $W: \mathcal{P} \ni P \longrightarrow W_{P} \in \Gamma\left(\otimes^{4} T^{*} M\right)$ which assigns to an operator $P$ the Weyl tensor of the Lorentzian metric $g_{P}$ associated to $P$. Its weight is $\omega=1$.

\section{The moments of normally hyperbolic operators}

\subsection{Mappings with simple transformation law}

The aim of this section is to generalize the notion of a conformal gauge invariant. Let $E$ be a vector bundle over a smooth n-dimensional manifold $M^{n}$ and let $\mathcal{P}$ be a set of normally hyperbolic operators on $E$ closed under conformal gauge transformation. Let $\mathcal{E}_{r s t u}$ be the bundles over $M^{n}$ defined in the previous section. For a mapping $T: \mathcal{P} \longmapsto \Gamma\left(\mathcal{E}_{r s t u}\right)$ the sections $T(P)$ and $e^{-2 \omega \varphi} A \cdot T\left(P_{\varphi, A}\right)$ now shall be allowed to differ by symmetric polynomials in first derivatives of $\varphi$. 
Definition 6.1 $A$ mapping $T: \mathcal{P} \longmapsto \Gamma\left(\mathcal{E}_{\text {rstu }}\right)$ has a simple transformation law with conformal weight $\omega$ if there exist linear maps

$$
\mathfrak{X}_{\nu} T: \mathcal{P} \longrightarrow \Gamma\left(S^{\nu}(T M) \otimes \mathcal{E}_{r s t u}\right) ; \quad 1 \leq \nu \leq N(T)
$$

such that

$$
T\left(P_{\varphi, A}\right)=e^{2 \omega \varphi} A^{-1} \cdot\left(T(P)+\sum_{\nu=1}^{N(T)}\left(\mathfrak{X}_{\nu} T\right)(P)(\underbrace{d \varphi, \ldots, d \varphi}_{\nu \text { times }})\right)
$$

holds for all $(\varphi, A) \in \mathcal{G}_{c}(E)$ and all $P \in \mathcal{P}$.

$\mathfrak{X} T:=\mathfrak{X}_{1} T: \mathcal{P} \longmapsto \Gamma\left(T M \otimes \mathcal{E}_{\text {rstu }}\right)$ is called the linear term of $T$.

Proposition 6.1 If $T: \mathcal{P} \longrightarrow \Gamma\left(\mathcal{E}_{\text {rstu }}\right)$ has a simple transformation law given by $\mathfrak{X}_{\nu} T: \mathcal{P} \longrightarrow \Gamma\left(S^{\nu}(T M) \otimes \mathcal{E}_{\text {rstu }}\right), \quad 1 \leq \nu \leq N(T)$, then the linear maps $\mathfrak{X}_{\nu} T$ have a simple transformation law and

$$
\mathfrak{X}_{\mu}\left(\mathfrak{X}_{\nu} T\right)=\left(\begin{array}{c}
\mu+\nu \\
\nu
\end{array}\right) \mathfrak{X}_{\mu+\nu} T ; \quad \mu+\nu \leq N(T) .
$$

In particular, all maps $\mathfrak{X}_{\nu} T$ in the transformation law of $T$ are uniquely determined by the linear term $\mathfrak{X T}$. More precisely, we have

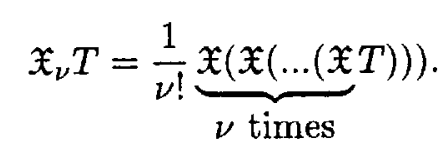

Proposition 6.2 Let $T: \mathcal{P} \longrightarrow \Gamma\left(\mathcal{E}_{r s t u}\right)$ have a simple transformation law. Then $T$ is a conformal gauge invariant if and only if $\mathfrak{X} T=0$.

We have the following rules for the linear term of a mapping with simple transformation law.

1. If $T, \widetilde{T}: \mathcal{P} \longrightarrow \Gamma\left(\mathcal{E}_{r s t u}\right)$ have a simple transformation laws with the same conformal weight $\omega$ and let $\lambda, \tilde{\lambda}$ be in $\mathbb{R}$ or $\mathbb{C}$ then $\lambda T+\widetilde{\lambda} \widetilde{T}$ has a simple transformation law with conformal weight $\omega$ and linear term

$$
\mathfrak{X}(\lambda T+\tilde{\lambda} \tilde{T})=\lambda \mathfrak{X} T+\tilde{\lambda} \mathfrak{X} \tilde{T} .
$$

2. $\mathfrak{X}$ commutes with contractions in the $T M$ - and $T^{*} M$-components of $\mathcal{E}_{\text {rstu }}$ and with contraction in the $E$ - and $E^{*}$-components of $\mathcal{E}_{r s t u}$.

3. If $T: \mathcal{P} \longrightarrow \Gamma\left(\mathcal{E}_{r s t u}\right)$ and $\widetilde{T}: \mathcal{P} \longrightarrow \Gamma\left(\mathcal{E}_{\text {tuij }}\right)$ have simple transformation laws with conformal weights $\omega$ and $\widetilde{\omega}$, respectively, then $T \otimes \widetilde{T}: \mathcal{P} \longmapsto \Gamma\left(\mathcal{E}_{r+t s+u k+i l+j}\right)$ has a simple transformation law with conformal weight $\omega+\widetilde{\omega}$. 
Now let $P: \Gamma(E) \longrightarrow \Gamma(E)$ be a normally hyperbolic operator and $\nabla^{P}: \Gamma(E) \longrightarrow$ $\Gamma\left(T^{*} M \otimes E\right)$ the covariant derivative occurring in the Weitzenböck formula of $P$. Then $\nabla^{P}$ induces a covariant derivative $\nabla^{P}$ in $\otimes^{k} E^{*} \otimes \otimes^{l} E$. Let $\widetilde{P}=P_{\varphi, A}$ be a conformal gauge transform of $P$. Then $\nabla^{\widetilde{P}}=A^{-1} \circ \nabla^{P} \circ A$ holds on $E$ (Proposition 14). The same formula is true for the induced covariant derivative on $\otimes^{k} E^{*} \otimes \bigotimes^{l} E$ where now $A$ acts as defined in (14). On the other hand $P$ defines a Lorentzian metric $g=g_{P}$. The associated Levi-Civita connection induces a covariant derivative $\nabla^{g}$ on $\otimes^{r} T^{*} M \otimes \otimes^{s} T M . \quad \nabla^{P}$ and $\nabla^{g}$ now define a covariant derivative on $\mathcal{E}_{r s t u}$ by the Leibniz rule. We also denote this by $\nabla^{P}$.

Definition 6.2 Let $T: \mathcal{P} \longrightarrow \Gamma\left(\mathcal{E}_{\text {rstu }}\right)$ be a mapping. Then the covariant derivative $\nabla T$ is defined to be the mapping

$$
\begin{gathered}
\nabla T: \mathcal{P} \longrightarrow \Gamma\left(T^{*} M \otimes \mathcal{E}_{r s t u}\right) \\
(\nabla T)(P):=\nabla^{P}(T(P)) .
\end{gathered}
$$

Remark If $T: \mathcal{P} \longrightarrow \Gamma\left(\mathcal{E}_{r s t u}\right)$ is a mapping with simple transformation law then in general $\nabla T: \mathcal{P} \longrightarrow \Gamma\left(T^{*} M \otimes \mathcal{E}_{\text {rstu }}\right)$ does not have a simple transformation law.

Therefore we are now going to modify this covariant derivative in order to obtain a derivative that preserves the property of being a map with simple transformation law.

\subsection{The conformal gauge derivative}

Let $g$ be a metric on $M^{n}$. Then the Schouten tensor $L_{g}$ of type $(2,0)$ associated to $g$ is given by

$$
L_{g}=\frac{1}{n-2}\left(\frac{1}{2(n-1)} R \cdot g-\mathrm{Ric}\right),
$$

where $R$ is the scalar curvature and Ric the Ricci tensor of $\left(M^{n}, g\right)$. We denote by the same symbol $L_{g}$ the Schouten map

$$
\begin{gathered}
L_{g}: T M \longrightarrow T^{*} M \\
L_{g}(X)(Y)=L_{g}(X, Y) .
\end{gathered}
$$

Definition 6.3 Let $T: \mathcal{P} \longrightarrow \Gamma\left(\mathcal{E}_{\text {rstu }}\right)$ be a mapping with simple transformation law and linear term $\mathfrak{X} T: \mathcal{P} \longmapsto \Gamma\left(T M \otimes \mathcal{E}_{\text {rstu }}\right)$. We denote by $\left.L\right\lrcorner \mathfrak{X} T$ the map

$$
\begin{gathered}
L\lrcorner \mathfrak{X} T: \mathcal{P} \longrightarrow \Gamma\left(T^{*} M \otimes \mathcal{E}_{r s t u}\right) \\
(L\lrcorner \mathfrak{X} T)(P)\left(X_{1}\right):=(\mathfrak{X T})(P)\left(L_{g}\left(X_{1}\right)\right)
\end{gathered}
$$

where $g$ is the Lorentzian metric induced by $P$.

Notation Here and in the following we only write those variables which are of special interest and not treated in the standard way. So $X_{1}, X_{2}, \ldots$ denotes the first, second etc. variable in $(T M)^{r}$ and $\sigma_{1}, \sigma_{2}, \ldots$ the first, second etc. variable in $\left(T^{*} M\right)^{s}$. 
Definition 6.4 Let $T: \mathcal{P} \longrightarrow \Gamma\left(\mathcal{E}_{\text {rstu }}\right)$ be a mapping with simple transformation law and linear term $\mathfrak{X} T$. Then the conformal gauge derivative $\mathfrak{C T}$ of $T$ is defined to be the following mapping

$$
\begin{gathered}
\mathfrak{C} T: \mathcal{P} \longrightarrow \Gamma\left(T^{*} M \otimes \mathcal{E}_{\text {rstu }}\right) \\
\mathfrak{C} T(P):=\nabla T(P)-(L \smile \mathfrak{X} T)(P) .
\end{gathered}
$$

Notation In the following we identify $T^{*} M \otimes \mathcal{E}_{r s t u}$ with $\mathcal{E}_{r+1} s k l$ where now the additional $T^{*} M$ is the first factor in $\mathcal{E}_{r+1} s k l$ etc.

The next aim is to describe the transformation law of the conformal gauge derivative of a mapping with simple transformation law.

Definition 6.5 Let be $\sigma \in T^{*} M, X \in T M$ and $g$ a metric on $M^{n}$. Then we define

$$
\begin{aligned}
K_{g}(\sigma, X): T M & \longrightarrow T M \\
Y & \longmapsto \sigma(X) Y+\sigma(Y) X-g(X, Y) \sigma^{\sharp}
\end{aligned}
$$

and

$$
\begin{gathered}
K_{g}(\sigma, X): T^{*} M \longrightarrow T^{*} M \\
\left(K_{g}(\sigma, X)(\omega)\right)(Y):=-\omega\left(K_{g}(\sigma, X)(Y)\right)
\end{gathered}
$$

where $\sigma^{\sharp}$ denotes the dual vector field of $\sigma$ with respect to $g$.

By direct calculations we obtain

Proposition 6.3 Let $g$ and $\widetilde{g}=e^{2 \varphi} g$.

1. The Schouten map $L_{\tilde{g}}$ of $\left(M^{n}, \tilde{g}\right)$ satisfies

$$
L_{\widetilde{g}}(X)=L_{g}(X)+\frac{1}{2}\|d \varphi\|^{2} g(X, \cdot)+\nabla_{X} d \varphi-d \varphi(X) \cdot d \varphi .
$$

2. The Levi-Civita connection $\tilde{\nabla}$ of $\left(M^{n}, \tilde{g}\right)$ on $T M$ as well as on $T^{*} M$ satisfies

$$
\widetilde{\nabla}_{X}=\nabla_{X}+K_{g}(d \varphi, X)
$$

where $\nabla$ is the Levi-Civita connection of $\left(M^{n}, g\right)$.

We now extend the map $K_{g}(\sigma, X)$ to $\mathcal{E}_{r s t u}$ in the following way. We set

$$
\begin{aligned}
& K_{g}(\sigma, X)\left(\sigma_{1} \otimes \ldots \otimes \sigma_{r} \otimes X_{1} \otimes \ldots \otimes X_{s} \otimes \Phi\right)= \\
& \left.\sum_{i} \sigma_{1} \otimes \ldots \otimes K_{g}(\sigma, X)\left(\sigma_{i}\right) \otimes \ldots \otimes \sigma_{r} \otimes X_{1} \otimes \ldots \otimes X_{s} \otimes \Phi\right) \\
& \left.+\sum_{j} \sigma_{1} \otimes \ldots \otimes \sigma_{r} \otimes X_{1} \otimes \ldots \otimes K_{g}(\sigma, X)\left(X_{j}\right) \otimes \ldots \otimes X_{s} \otimes \Phi\right)
\end{aligned}
$$

where $\Phi$ is in $\otimes^{k} E^{*} \otimes \bigotimes^{l} E$. In particular, $K_{g}(\sigma, X) \equiv 0$ on $\mathcal{E}_{r s t u}$ if $r=s=0$. 
Proposition 6.4 Let $P$ be a normally hyperbolic operator on $E$ and $\widetilde{P}=P_{\varphi, A}$ a conformal gauge transform of $P$. Furthermore let $\nabla^{P}$ and $\nabla^{\widetilde{P}}$ be the induced derivatives on $\mathcal{E}_{\text {rstu }}$. Then we have

$$
\nabla^{\widetilde{P}}=A^{-1} \circ\left(\nabla^{P}+K_{g}(d \varphi, \cdot)\right) \circ A .
$$

Definition 6.6 Let $T: \mathcal{P} \longrightarrow \Gamma\left(\mathcal{E}_{\text {rstu }}\right)$ be a mapping. Then the mapping $K T$ is defined by

$$
\begin{gathered}
K T: \mathcal{P} \longrightarrow \Gamma\left(\mathcal{E}_{r+1 s+1 k l}\right) \\
(K T)(P)\left(X_{1}, \sigma_{1}\right):=K_{g}\left(\sigma_{1}, X_{1}\right)(T(P))
\end{gathered}
$$

where $g$ is the Lorentzian metric induced by $P$.

Let $\Sigma \in T M \otimes T^{*} M$ be the Kronecker tensor on $M^{n}$, i.e. $\Sigma(\sigma, X):=\sigma(X)$.

Definition 6.7 The extension of a mapping $T: \mathcal{P} \longrightarrow \Gamma\left(\mathcal{E}_{r s t u}\right)$ (by the Kronecker tensor) is defined to be the mapping

$$
\begin{aligned}
E T: \mathcal{P} & \longrightarrow \Gamma\left(T M \otimes T^{*} M \otimes \mathcal{E}_{r s t u}\right) \\
P & \longmapsto \Sigma \otimes T(P)
\end{aligned}
$$

Theorem 6.1 Let $T: \mathcal{P} \longrightarrow \Gamma\left(\mathcal{E}_{\text {rstu }}\right)$ be a mapping with simple transformation law and conformal weight $\omega$. Then the conformal gauge derivative

$$
\mathfrak{C} T: \mathcal{P} \longrightarrow \Gamma\left(T^{*} M \otimes \mathcal{E}_{r s t u}\right)
$$

of $T$ has a simple transformation law with conformal weight $\omega$ and linear term

$$
\begin{gathered}
\mathfrak{X}(\mathfrak{C} T): \mathcal{P} \longrightarrow \Gamma\left(\mathcal{E}_{r+1 s+1 t u}\right) \\
\mathfrak{X}(\mathfrak{C} T)=\mathfrak{C} \mathfrak{X} T+K T+2 \omega E T .
\end{gathered}
$$

Proof. Let $\widetilde{P}=P_{\varphi, A}$ be a conformal gauge transform of a normally hyperbolic operator $P$ and $g$ and $\tilde{g}$ the Lorentzian metrics on $M^{n}$ associated to $P$ and $\widetilde{P}$, respectively. By Definition 6.2 we have $\mathfrak{C} T(\widetilde{P})=\nabla T(\widetilde{P})-(L\lrcorner \mathfrak{X} T)(\widetilde{P})$. The first term of the r. h. s. equals

$$
\begin{aligned}
\nabla T & (\widetilde{P})\left(X_{1}\right)=\nabla_{X_{1}}^{\widetilde{P}}(T \widetilde{P}) \\
& =A^{-1}\left(\nabla_{X_{1}}^{P}+K_{g}\left(d \varphi, X_{1}\right)\right) A(T \widetilde{P}) \\
& =A^{-1}\left(\nabla_{X_{1}}^{P}+K_{g}\left(d \varphi, X_{1}\right)\right) e^{2 \omega \varphi}\left(T P+\sum_{\nu=1}^{N} \mathfrak{X}_{\nu} T(P)(\underbrace{d \varphi, \ldots, d \varphi}_{\nu \text { times }})\right) \\
& =e^{2 \omega \varphi} A^{-1}\left(\nabla_{X_{1}}^{P}(T P)+\sum_{\nu=1}^{N} \nabla_{X_{1}}^{P}\left(\mathfrak{X}_{\nu} T(P)(d \varphi, \ldots, d \varphi)\right)\right.
\end{aligned}
$$




$$
\begin{aligned}
& +2 \omega d \varphi\left(X_{1}\right) \cdot\left(T P+\sum_{\nu=1}^{N} \mathfrak{X}_{\nu} T(P)(d \varphi, \ldots, d \varphi)\right) \\
& \left.+K_{g}\left(d \varphi, X_{1}\right)\left(T P+\sum_{\nu=1}^{N} \mathfrak{X}_{\nu} T(P)(d \varphi, \ldots, d \varphi)\right)\right) \\
=e^{2 \omega \varphi} A^{-1}\left(\nabla_{X_{1}}^{P}(T P)\right. & \\
+ & \sum_{\nu=1}^{N}\left(\nabla_{X_{1}}^{P} \mathfrak{X}_{\nu} T(P)\right)(d \varphi, \ldots, d \varphi)+\sum_{\nu=1}^{N} \mathfrak{X}_{\nu} T(P)(\nabla_{X_{1}}^{g} d \varphi, \underbrace{d \varphi, \ldots, d \varphi}_{\nu-1 \text { times }}) \\
& +2 \omega E T(P)\left(X_{1}, d \varphi\right)+2 \omega \sum_{\nu=1}^{N}\left(E \mathfrak{X}_{\nu} T\right)(P)(X_{1}, \underbrace{d \varphi, \ldots, d \varphi}_{\nu+1 \text { times }}) \\
& \left.+K T(P)\left(X_{1}, d \varphi\right)+\sum_{\nu=1}^{N}\left(K \mathfrak{X}_{\nu} T\right)(P)(X_{1}, \underbrace{d \varphi, \ldots, d \varphi}_{\nu+1 \text { times }})\right)
\end{aligned}
$$

Since we are only interested in the transformation law and the linear term of $\mathfrak{C} T$ it suffices to consider this result modulo terms containing only powers of first derivatives of $\varphi$ of at least second order. Hence,

$$
\begin{aligned}
\nabla T(\widetilde{P})\left(X_{1}\right) \equiv e^{2 \omega \varphi} A^{-1} & \left(\nabla_{X_{1}}^{P}(T P)\right. \\
& +\nabla_{X_{1}}^{P} \mathfrak{X T}(P)(d \varphi)+\sum_{\nu=1}^{N} \nu \mathfrak{X}_{\nu} T(P)\left(\nabla_{X_{1}}^{g} d \varphi, d \varphi, \ldots, d \varphi\right) \\
& \left.+2 \omega E T(P)\left(X_{1}, d \varphi\right)+K T(P)\left(X_{1}, d \varphi\right)\right) .
\end{aligned}
$$

Now we treat the second term of $\mathfrak{C} T$ in the same manner.

$$
\begin{aligned}
\left(L_{\perp} \mathfrak{X} T\right)(\widetilde{P})\left(X_{1}\right)=\mathfrak{X} T(\widetilde{P})\left(L_{\tilde{g}}\left(X_{1}\right)\right)=\mathfrak{X}_{1} T(\widetilde{P})\left(L_{\tilde{g}}\left(X_{1}\right)\right) & \\
= & e^{2 \omega \varphi} A^{-1}\left(\mathfrak{X}_{1} T(P)\left(L_{\tilde{g}}\left(X_{1}\right)\right)+\sum_{\nu=1}^{N} \mathfrak{X}_{\nu} \mathfrak{X}_{1} T(P)(L_{\tilde{g}}\left(X_{1}\right), \underbrace{d \varphi, \ldots, d \varphi}_{\nu \text { times }})\right) \\
= & e^{2 \omega \varphi} A^{-1}\left(\mathfrak{X}_{1} T(P)\left(L_{\tilde{g}}\left(X_{1}\right)\right)+\sum_{\nu=1}^{N-1}(\nu+1) \mathfrak{X}_{\nu+1} T(P)\left(L_{\tilde{g}}\left(X_{1}\right), d \varphi, \ldots, d \varphi\right)\right) \\
= & e^{2 \omega \varphi} A^{-1}\left(\mathfrak{X} T(P)\left(L_{g}\left(X_{1}\right)+\frac{1}{2}\|d \varphi\|^{2} g\left(X_{1}, \cdot\right)+\nabla_{X_{1}}^{g} d \varphi-d \varphi\left(X_{1}\right) \cdot d \varphi\right)\right. \\
& \quad+\sum_{\nu=1}^{N-1}(\nu+1) \mathfrak{X}_{\nu+1} T(P)\left(L_{g}\left(X_{1}\right)+\frac{1}{2}\|d \varphi\|^{2} g\left(X_{1}, \cdot\right)+\right. \\
& \nabla_{X_{1}}^{g} d \varphi-d \varphi\left(X_{1}\right) \cdot d \varphi, \underbrace{d \varphi, \ldots, d \varphi}_{\nu \text { times }})
\end{aligned}
$$




$$
\begin{aligned}
\equiv e^{2 \omega \varphi} A^{-1} & ((L\lrcorner \mathfrak{X} T)(P)\left(X_{1}\right)+\mathfrak{X} T(P)\left(\nabla_{X_{1}}^{g} d \varphi\right)+2 \mathfrak{X}_{2} T(P)\left(L_{g}\left(X_{1}\right), d \varphi\right) \\
& \left.+\sum_{\nu=1}^{N-1}(\nu+1) \mathfrak{X}_{\nu+1} T(P)(\nabla_{X_{1}}^{g} d \varphi, \underbrace{d \varphi, \ldots, d \varphi}_{\nu \text { times }})\right) \\
=e^{2 \omega \varphi} A^{-1} & \left.((L\lrcorner \mathfrak{X} T)(P)\left(X_{1}\right)+(L\lrcorner \mathfrak{X}(\mathfrak{X} T)\right)(P)\left(X_{1}, d \varphi\right) \\
& \left.+\sum_{\nu=0}^{N-1}(\nu+1) \mathfrak{X}_{\nu+1} T(P)(\nabla_{X_{1}}^{g} d \varphi, \underbrace{d \varphi, \ldots, d \varphi}_{\nu \text { times }})\right)
\end{aligned}
$$

where the equivalence is defined as above. The result now follows by comparing both terms.

We have the following rules for the conformal gauge derivative.

1. If $T, \widetilde{T}: \mathcal{P} \longrightarrow \Gamma\left(\mathcal{E}_{\text {rstu }}\right)$ have simple transformation laws with the same conformal weight $\omega$ and if $\lambda, \tilde{\lambda}$ is in $\mathbb{R}$ or $\mathbb{C}$ then

$$
\mathfrak{C}(\lambda T+\tilde{\lambda} \tilde{T})=\lambda \mathfrak{C} T+\tilde{\lambda} \mathfrak{C} \tilde{T}
$$

2. $\mathfrak{C}$ commutes with contractions in the $T M$ - and $T^{*} M$-components of $\mathcal{E}_{r s t u}$ and with contraction in the $E$ - and $E^{*}$-components of $\mathcal{E}_{\text {rstu }}$.

3. If $T: \mathcal{P} \longrightarrow \Gamma\left(\mathcal{E}_{r s t u}\right)$ and $\widetilde{T}: \mathcal{P} \longrightarrow \Gamma\left(\mathcal{E}_{\tilde{r} \tilde{s} \tilde{t} \tilde{u}}\right)$ have a simple transformation laws then

$$
\mathfrak{C}(T \otimes \tilde{T})=\mathfrak{C}(T) \otimes \widetilde{T}+T \otimes \mathfrak{C}(\tilde{T}) .
$$

4. If $f \in C^{\infty}\left(M^{n}\right)$ is a smooth function and $T: \mathcal{P} \longrightarrow \Gamma\left(\mathcal{E}_{r s t u}\right)$ a mapping with simple transformation law then the mapping $f T$ defined by

$$
\begin{aligned}
f T: \mathcal{P} & \longrightarrow \Gamma\left(\mathcal{E}_{r s t u}\right) \\
P & \longmapsto f \cdot T(P)
\end{aligned}
$$

has a simple transformation law and its conformal gauge derivative satisfies

$$
\mathfrak{C}(f T)=d f \otimes T+f \mathfrak{C} T .
$$

Definition 6.8 Let $T: \mathcal{P} \longrightarrow \Gamma\left(\mathcal{E}_{r s t u}\right)$ be a mapping with simple transformation law. Fix a set of $T^{*} M$-components of $\mathcal{E}_{\text {rstu }}$. Then the symmetrization $S T$ of $T$ is defined to be the mapping

$$
\begin{aligned}
S T: \mathcal{P} & \longrightarrow \Gamma\left(\mathcal{E}_{\text {rstu }}\right) \\
P & \longmapsto S(T(P))
\end{aligned}
$$

where $S(T(P))$ is the symmetrization of $T(P)$ with respect to the chosen set of $T^{*} M$ components. 
Obviously, $S T$ has again a simple transformation law and $\mathfrak{X S T}=S \mathfrak{X} T$.

Proposition 6.5 Let $T$ be as above. Then the extension ET has a simple transformation law and satisfies

1. $(\mathfrak{X E T}(P))\left(\sigma_{1}\right)=E\left(\mathfrak{X} T(P)\left(\sigma_{1}\right)\right)$ for all $\sigma_{1} \in T^{*} M$ and any $P \in \mathcal{P}$

2. $\mathfrak{C}_{X} E T=E \mathfrak{C}_{X} T$ for all vector fields $X \in \mathfrak{X}\left(M^{n}\right)$.

In particular, we have

3. $S(\mathfrak{C E T})=S(E \mathfrak{C} T)$

where $S$ is the symmetrization over the additional $T^{*} M$-components arising from the derivation and the extension of $T$.

Notation Let $U_{1}, U_{2} \in S^{k}\left(T^{*} M\right) \otimes \mathcal{E}_{\text {rstu }}$ and $V \in S^{l}\left(T^{*} M\right)(l \leq k)$ be symmetric tensors. Then we note $U_{1} \equiv U_{2} \bmod V$ if there exist a symmetric tensor $W \in$ $S^{k-l}\left(T^{*} M\right) \otimes \mathcal{E}_{r s t u}$ such that $U_{1}-U_{2}=V \circ W$ where $\circ$ denotes the symmetric tensor product.

Proposition 6.6 Let $T$ be as above. Then $K T$ has a simple transformation law and satisfies

1. $(\mathfrak{X K T}(P))\left(\sigma_{1}\right)=K_{g}\left(\mathfrak{X} T(P)\left(\sigma_{1}\right)\right)$ for all $\sigma_{1} \in T^{*} M$ and any $P \in \mathcal{P}$ with associated metric $g$.

2. $\left(\mathfrak{C}_{X} K T\right)(P)=K_{g}\left(\mathfrak{C}_{X} T(P)\right)$ for all vector fields $X \in \mathfrak{X}\left(M^{n}\right)$ and any $P \in \mathcal{P}$ with associated metric $g$.

In particular, after symmetrization over the additional $T^{*} M$-components we obtain

3. $S(K \mathfrak{C} T) \equiv-2 S(E \mathfrak{C} T)+S(\mathfrak{C} K T) \bmod g$.

Let $T: \mathcal{P} \longrightarrow \Gamma\left(\mathcal{E}_{r s t u}\right)$ be a mapping with simple transformation law. We consider the $k$-fold conformal gauge derivative

$$
\mathfrak{C}^{k} T:=\underbrace{\mathfrak{C C} \ldots \mathfrak{C}}_{k \text { times }} T: \mathcal{P} \longrightarrow \Gamma\left(\otimes^{k} T^{*} M \otimes \mathcal{E}_{r s t u}\right)
$$

and symmetrize it with respect to the $k$ additional $T^{*} M$-components to obtain a mapping

$$
S\left(\mathfrak{C}^{k} T\right): \mathcal{P} \longrightarrow \Gamma\left(S^{k} T^{*} M \otimes \mathcal{E}_{r s t u}\right)
$$

Theorem 6.2 Let $T: \mathcal{P} \longrightarrow \Gamma\left(\mathcal{E}_{\text {rstu }}\right)$ be a conformal gauge invariant. Then the linear term of $S\left(\mathfrak{C}^{k} T\right)$ satisfies

$$
\mathfrak{X}\left(S \mathfrak{C}^{k} T\right) \equiv k(2 \omega-k+1) S\left(E \mathfrak{C}^{k-1} T\right)+k S\left(\mathfrak{C}^{k-1} K T\right) \bmod g
$$

where $S$ is the symmetrization with respect to the $k$ additional $T^{*} M$-components. 
Theorem 6.3 Let $\mathcal{P}$ be as above. Fix $\omega \in \mathbb{R} ; k \in \mathbb{N}, k \geq 1$ and $r, s, t, u \in \mathbb{N}$. There exist "universal" mappings

$$
\begin{aligned}
\mathcal{K}^{(\nu, k)}: \mathcal{P} & \longrightarrow \Gamma\left(\left(T^{*} M\right)^{\nu} \otimes \operatorname{Hom}\left(\left(T^{*} M\right)^{k-\nu} \otimes \mathcal{E}_{r s t u}\right)\right. \\
P & \longmapsto \mathcal{K}_{P}^{(\nu, k)} \quad(1 \leq \nu \leq k)
\end{aligned}
$$

(depending only on $\omega$ and $r, s, t, u$ ) such that

$$
\mathfrak{C}^{k} T(P)=\nabla^{k} T(P)+\sum_{\nu=1}^{k} \mathcal{K}_{P}^{(\nu, k)}\left(\nabla^{k-\nu} T(P)\right)
$$

holds for all conformal gauge invariants $T: \mathcal{P} \longrightarrow \Gamma\left(\mathcal{E}_{\text {rstu }}\right)$ of weight $\omega$.

\subsection{The trace free part of mappings with simple transformation law}

Let $\Phi \in S^{k}\left(T^{*} M\right) \otimes \mathcal{E}_{r s t u}$ be a symmetric tensor. Then there exists a unique decomposition $\Phi=\Phi_{1}+g \circ \Phi_{2}$ such that $\Phi_{1} \in S^{k}\left(T^{*} M\right) \otimes \mathcal{E}_{r s t u}, \Phi_{2} \in S^{k-2}\left(T^{*} M\right) \otimes \mathcal{E}_{r s t u}$ and the trace of $\Phi_{1}$ vanishes, i.e. Trace $\left(\Phi_{1}\right)=0$. The tensor $\Phi_{1}$ is called the trace free part of $\Phi$ and is denoted by $\mathcal{T} \Phi$. On a certain condition given by the following theorem the trace free part of a mapping with simple transformation law is a conformal gauge invariant.

Theorem 6.4 Let $T: \mathcal{P} \longrightarrow \Gamma\left(S^{k}\left(T^{*} M\right) \otimes \mathcal{E}_{\text {rstu }}\right)$ be a mapping with simple transformation law. If $\mathfrak{X} T \equiv 0 \bmod g$ then $\mathcal{T} T$ is a conformal gauge invariant.

Proof. Let $T=\mathcal{T} T+g \circ \hat{T}$ be the unique decomposition of $T$ into a trace free and a trace part. Then one can prove that $\hat{T}$ is given by $T$ in the form

$$
\hat{T}=\sum_{l=1}^{\left[\frac{k}{2}\right]} c_{l} g^{l-1} \circ \operatorname{Trace}_{g}^{l} T
$$

where $c_{l}$ are certain constants. Since $\mathrm{T}$ has simple transformation law with conformal weight $\omega$ and $g$ has conformal weight 1

$$
g^{l-1} \circ \operatorname{Trace}_{g}^{l} T: \mathcal{P} \longrightarrow \Gamma\left(S^{k-2}\left(T^{*} M\right) \otimes \mathcal{E}_{r s t u}\right)
$$

has simple transformation law with conformal weight $\omega-1$ and the linear term is

$$
\mathfrak{X}\left(g^{l-1} \circ \operatorname{Trace}_{g}^{l} T\right)=g^{l-1} \circ \operatorname{Trace}_{g}^{l}(\mathfrak{X} T) .
$$

Hence, by (15) $\hat{T}$ has simple transformation law with conformal weight $\omega-1$ and linear term

$$
\mathfrak{X}(\hat{T})=\sum_{l=1}^{\left[\frac{k}{2}\right]} c_{l} g^{l-1} \circ \operatorname{Trace}_{g}^{l}(\mathfrak{X} T) .
$$


It follows that $\mathcal{T} T \longrightarrow \Gamma\left(S^{k}\left(T^{*} M\right) \otimes \mathcal{E}_{\text {rstu }}\right)$ has simple transformation law with conformal weight $\omega$ and linear term

$$
\mathfrak{X}(\mathcal{T} T)=\mathfrak{X}(T)-g \circ \mathfrak{X}(\hat{T}) .
$$

Since Trace $(\mathfrak{X}(\mathcal{T} T))=\mathfrak{X}(\operatorname{Trace} \mathcal{T} T)=0, \mathfrak{X}(\mathcal{T} T)$ is the trace free part of $\mathfrak{X}(T)$, i.e.

$$
\mathcal{T}(\mathfrak{X} T)=\mathfrak{X}(\mathcal{T} T) .
$$

By assumption $\mathfrak{X} T \equiv 0 \bmod g$. Hence, $\mathfrak{X}(\mathcal{T} T)=\mathcal{T}(\mathfrak{X} T)=0$. Therefore, accordingly to Proposition $6.2 \mathcal{T} T$ is a conformal gauge invariant.

\subsection{Kernels with simple transformation law}

Now we extend the notion of mappings with simple transformation law to mappings from $\mathcal{P}$ into the space of smooth sections of a certain vector bundle over $M^{n} \times M^{n}$. These are called kernels. Let $M^{n}, E, \mathcal{P}$ be as in the preceding section. We consider the product manifold $M^{n} \times M^{n}$ and denote by $p_{1}$ and $p_{2}$ the projection onto the first and second factor, respectively.

Definition 6.9 Denote by $\mathcal{F}_{r s k l}$ the bundle $\otimes^{r} p_{1}^{*}\left(T^{*} M\right) \otimes \bigotimes^{s} p_{2}^{*}\left(T^{*} M\right) \otimes \bigotimes^{k} p_{1}^{*}(T M) \otimes$ $\otimes^{l} p_{2}^{*}(T M) \otimes E^{*} \otimes E$ over $M^{n} \times M^{n}$.

Let $A \in \Gamma(\operatorname{Aut}(E))$ be an invertible homomorphism. We define an action of $A$ on $\mathcal{F}_{r s k l}$ by

$$
A \cdot(\Psi \otimes f \otimes e)_{(x, y)}=(\Psi \otimes A(x) \cdot f \otimes A(y) \cdot e)_{(x, y)}
$$

where $\Psi \in \otimes^{r} p_{1}^{*}\left(T^{*} M\right) \otimes \otimes^{s} p_{2}^{*}\left(T^{*} M\right) \otimes \otimes^{k} p_{1}^{*}(T M) \otimes \otimes^{l} p_{2}^{*}(T M)$ and $A(x) \cdot f=$ $\left(A(x)^{-1}\right)^{*}(f), A(y) \cdot e=A(y)(e)$. If we identify $\Phi_{(x, y)} \in\left(E^{*} \otimes E\right)_{(x, y)}$ in the standard way with the homomorphism $\Phi(x, y) \in \operatorname{Hom}\left(E_{x}, E_{y}\right)$ the action $A \cdot \Phi_{(x, y)}$ transforms into $A(y) \circ \Phi(x, y) \circ A(x)^{-1}$.

Definition 6.10 A kernel $T: \mathcal{P} \longmapsto \Gamma\left(\mathcal{F}_{\text {rskl }}\right)$ has a simple transformation law with conformal weight $\omega$ if there exist linear maps

$$
\begin{gathered}
\mathfrak{X}_{(\mu, \nu)}: \mathcal{P} \longrightarrow \Gamma\left(S^{\mu} p_{1}^{*}(T M) \otimes S^{\nu} p_{2}^{*}(T M) \otimes \mathcal{F}_{r s k l}\right) \\
0 \leq \mu \leq M(T), \quad 0 \leq \nu \leq N(T), \mu+\nu \geq 1
\end{gathered}
$$

such that

$$
\begin{aligned}
& T\left(P_{\varphi, A}\right)_{(x, y)}=e^{\omega(\varphi(x)+\varphi(y))} A^{-1} \cdot(T(P) \\
& \quad+\sum_{\substack{\nu=0, \ldots, N(T) \\
\mu=0, \ldots, M(T) \\
\mu, \nu \geq 1}}\left(\mathfrak{X}_{(\mu, \nu)} T\right)(P)(\underbrace{p_{1}^{*}(d \varphi), \ldots, p_{1}^{*}(d \varphi)}_{\mu \text { times }}, \underbrace{\left.\left.p_{2}^{*}(d \varphi), \ldots, p_{2}^{*}(d \varphi)\right)\right)}_{\nu \text { times }})
\end{aligned}
$$

holds for all $(\varphi, A) \in \mathcal{G}_{c}(E)$ and all $P \in \mathcal{P}$.

$\mathfrak{X}^{(1)} T:=\mathfrak{X}_{(1,0)} T: \mathcal{P} \longmapsto \Gamma\left(p_{1}^{*}(T M) \otimes \mathcal{F}_{r s k l}\right)$ and $\mathfrak{X}^{(2)} T:=\mathfrak{X}_{(0,1)} T: \mathcal{P} \longmapsto \Gamma\left(p_{2}^{*}(T M) \otimes\right.$ $\left.\mathcal{F}_{\text {rskl }}\right)$ are called the linear terms of $T$. 
Notation Similarly as above we identify $p_{1}^{*}\left(T^{*} M\right) \otimes \mathcal{F}_{r s k l}$ with $\mathcal{F}_{r+1 \text { skl }}$ etc.

Proposition 6.7 If $T: \mathcal{P} \longrightarrow \Gamma\left(\mathcal{F}_{\text {rskl }}\right)$ has a simple transformation law given by $\mathfrak{X}_{(\mu, \nu)} T: \mathcal{P} \longrightarrow \Gamma\left(\mathcal{F}_{r s k+\mu l+\nu}\right) ; 0 \leq \mu \leq M(T), \quad 0 \leq \nu \leq N(T), \mu+\nu \geq 1$ then the maps $X_{(\mu, \nu)}$ have a simple transformation law and

$$
\begin{aligned}
\mathfrak{X}_{(\alpha, \beta)}\left(\mathfrak{X}_{(\mu, \nu)} T\right)=\left(\begin{array}{c}
\mu+\alpha \\
\mu
\end{array}\right)\left(\begin{array}{c}
\nu+\beta \\
\nu
\end{array}\right) \mathfrak{X}_{(\mu+\alpha, \nu+\beta)} T ; \\
\mu+\alpha \leq M(T), \nu+\beta \leq N(T) .
\end{aligned}
$$

Now we consider again the Schouten map $L_{g}: T M \longrightarrow T^{*} M$ and define

$$
\begin{aligned}
& L_{g}^{(i)}: p_{i}^{*} T M \longrightarrow p_{i}^{*}\left(T^{*} M\right) \quad i=1,2 \\
& L_{g}^{(i)}:=p_{i}^{*} L_{g}
\end{aligned}
$$

Notation We denote by $\left.L^{(i)}\right\lrcorner \mathfrak{X}^{(i)} T, i=1,2$ the mappings

$$
\begin{gathered}
\left.L^{(i)}\right\lrcorner \mathfrak{X}^{(i)} T: \mathcal{P} \longrightarrow \Gamma\left(p_{i}^{*}\left(T^{*} M\right) \otimes \mathcal{F}_{r s k l}\right) \\
\left.\left(L^{(i)}\right\lrcorner \mathfrak{X}^{(i)} T\right)(P)\left(X_{1}\right)=\left(\mathfrak{X}^{(i)} T\right)(P)\left(L_{g}^{(i)}\left(X_{1}\right)\right) \quad i=1,2
\end{gathered}
$$

where $g$ is the Lorentzian metric induced by $P$.

Definition 6.11 Let $T: \mathcal{P} \longrightarrow \Gamma\left(\mathcal{F}_{\text {rskl }}\right)$ be a kernel with simple transformation law and linear terms $\mathfrak{X} T$. Then the partial conformal gauge derivatives $\mathfrak{C}^{(i)} T$ of $T$ are defined to be the mappings

$$
\begin{gathered}
\mathfrak{C}^{(i)} T: \mathcal{P} \longrightarrow \Gamma\left(p_{i}^{*}\left(T^{*} M\right) \otimes \mathcal{F}_{r s k l}\right) \\
\left.\mathfrak{C}^{(i)} T(P):=\nabla^{(i)} T(P)-\left(L^{(i)}\right\lrcorner \mathfrak{X}^{(i)} T\right)(P),
\end{gathered}
$$

where $\nabla^{(i)} T(P)$ is the covariant derivative defined by the Levi-Civita connection of $g_{P}$ and $\nabla^{P}$ in the ith component of the product $M \times M$.

Definition 6.12 Let $g$ be a metric on $M^{n}$ and $\sigma \in p_{1}^{*}\left(T^{*} M\right), X \in p_{1}^{*}(T M)$. Then we define a map $K_{g}^{(1)}(\sigma, X)$ on $\mathcal{F}_{\text {rskl }}$ by

$$
\begin{aligned}
& K_{g}^{(1)}(\sigma, X)\left(\sigma_{1} \otimes \ldots \sigma_{r} \otimes \tau_{1} \otimes \ldots \tau_{s} \otimes X_{1} \otimes \ldots X_{k} \otimes Y_{1} \otimes \ldots X_{l} \otimes f \otimes e\right) \\
& =\sum_{i=1}^{r} \sigma_{1} \otimes \ldots \otimes\left(p_{1}^{*} K_{g}\right)(\sigma, X) \sigma_{i} \otimes \ldots \otimes \sigma_{r} \otimes \tau_{1} \otimes \ldots \otimes f \otimes e \\
& \quad+\sum_{j=1}^{k} \sigma_{1} \otimes \ldots \otimes X_{1} \otimes \ldots \otimes\left(p_{1}^{*} K_{g}\right)(\sigma, X) X_{j} \otimes \ldots \otimes X_{k} \otimes \ldots \otimes f \otimes e .
\end{aligned}
$$

In particular, $K_{g}^{(1)}(\sigma, X) \equiv 0$ on $\mathcal{F}_{r s k l}$ if $r=k=0$.

Now let $T: \mathcal{P} \longrightarrow \Gamma\left(\mathcal{F}_{\text {rskl }}\right)$ be a kernel with simple transformation law. We set

$$
\begin{gathered}
K^{(1)} T: \mathcal{P} \longrightarrow \Gamma\left(\mathcal{F}_{r+1 s k+1 l}\right) \\
\left(K^{(1)} T\right)(P)\left(X_{1}, \sigma_{1}\right)=K_{g}^{(1)}\left(\sigma_{1}, X_{1}\right)(T(P)) .
\end{gathered}
$$

Similarly we define $K^{(2)} T: \mathcal{P} \longrightarrow \Gamma\left(\mathcal{F}_{r s+1 k l+1}\right)$. 
Let $\Sigma \in T^{*} M \otimes T M$ be the Kronecker tensor as defined above.

Definition 6.13 Let $T$ be as in the preceding definition. We define

$$
\begin{aligned}
& E^{(i)} T: \mathcal{P} \longrightarrow \Gamma\left(p_{i}^{*}\left(T^{*} M\right) \otimes p_{i}^{*}(T M) \otimes \mathcal{F}_{r s k l}\right) \\
& E^{(i)} T(P):=p_{1}^{*} \Sigma \otimes T(P) .
\end{aligned}
$$

Similarly to Theorem 6.1 one proves

Theorem 6.5 Let $T: \mathcal{P} \longrightarrow \Gamma\left(\mathcal{F}_{\text {rskl }}\right)$ be a kernel with simple transformation law and conformal weight $\omega$. Then the partial conformal gauge derivatives $\mathfrak{C}^{(1)} T$ and $\mathfrak{C}^{(2)} T$ have simple transformation laws with conformal weight $\omega$ and their linear terms satisfy

$$
\begin{array}{cl}
\mathfrak{X}^{(i)}\left(\mathfrak{C}^{(i)} T\right)=\mathfrak{C}^{(i)}\left(\mathfrak{X}^{(i)} T\right)+K^{(i)} T+\omega E^{(i)} T & \text { for } i=1,2 \\
\mathfrak{X}^{(i)}\left(\mathfrak{C}^{(j)} T\right)=\mathfrak{C}^{(j)}\left(\mathfrak{X}^{(i)} T\right) & \text { if } i \neq j .
\end{array}
$$

We have the following rules for the partial conformal gauge derivatives.

1. If $T, \widetilde{T}: \mathcal{P} \longrightarrow \Gamma\left(\mathcal{F}_{r s k l}\right)$ have simple transformation laws with the same conformal weight $\omega$ and if $\lambda, \tilde{\lambda}$ is in $\mathbb{R}$ or $\mathbb{C}$ then

$$
\mathfrak{C}^{(i)}(\lambda T+\tilde{\lambda} \tilde{T})=\lambda \mathfrak{C}^{(i)} T+\tilde{\lambda} \mathfrak{C}^{(i)} \tilde{T}, i=1,2 .
$$

2. $\mathfrak{C}^{(i)}$ commutes with contractions in the $p_{j}^{*}(T M)$ - and $p_{j}^{*}\left(T^{*} M\right)$-components of $\mathcal{F}_{r s k l}, i=1,2$ and with contraction in the $E$ - and $E^{*}$-component of $\mathcal{F}_{r s k l}$.

3. If $f \in C^{\infty}\left(M^{n} \times M^{n}\right)$ is a smooth function and $T: \mathcal{P} \longrightarrow \Gamma\left(\mathcal{F}_{r s k l}\right)$ a kernel with simple transformation law then the mapping $f T$ defined by

$$
\begin{aligned}
f T: \mathcal{P} & \longrightarrow \Gamma\left(\mathcal{F}_{r s k l}\right) \\
P & \longmapsto f \cdot T(P)
\end{aligned}
$$

has a simple transformation law and its partial conformal gauge derivatives satisfy

$$
\mathfrak{C}^{(i)}(f T)=d f \otimes T+f \mathfrak{C}^{(i)} T, i=1,2 .
$$

4. If $T$ is as above, then

$$
\mathfrak{C}^{(1)} \mathfrak{C}^{(2)} T=\mathfrak{C}^{(2)} \mathfrak{C}^{(1)} T
$$

In the following $T: \mathcal{P} \longrightarrow \Gamma\left(\mathcal{F}_{r s k l}\right)$ is a kernel with simple transformation law.

Definition 6.14 We fix a set of $p_{i}^{*}\left(T^{*} M\right)$-components of $\mathcal{F}_{r s k l},(i=1,2)$ and denote by $S^{(i)} T$ the symmetrization of $T$ with respect to these components.

Direct calculations show that the following commutation rules hold. 
Proposition $6.8 S^{(i)} T$ is a kernel with simple transformation law and linear terms

$$
\mathfrak{X}^{(j)} S^{(i)} T=S^{(i)} \mathfrak{X}^{(j)} T,(i=1,2) .
$$

If $i \neq j$ then

$$
\begin{aligned}
S^{(i)} \mathfrak{C}^{(j)} T & =\mathfrak{C}^{(j)} S^{(i)} T \\
S^{(i)} E^{(j)} T & =E^{(j)} S^{(i)} T \\
S^{(i)} K^{(j)} T & =K^{(j)} S^{(i)} T .
\end{aligned}
$$

Proposition 6.9 The extensions $E^{(i)} T$ are kernels with simple transformation laws and satisfy

1. $\mathfrak{X}^{(i)} E^{(j)} T=E^{(j)} \mathfrak{X}^{(i)} T$ if $i \neq j$

2. $\left(\mathfrak{X}^{(i)} E^{(i)} T(P)\right)\left(\sigma_{1}\right)=E^{(i)}\left(\mathfrak{X}^{(i)} T(P)\left(\sigma_{1}\right)\right),(i=1,2)$ for all $\sigma_{1} \in p_{i}^{*}\left(T^{*} M\right)$ and any $P \in \mathcal{P}$

3. $\mathfrak{C}^{(i)} E^{(j)} T=E^{(j)} \mathfrak{C}^{(i)} T$ if $i \neq j$

4. $\mathfrak{C}_{X}^{(i)} E^{(i)} T=E^{(i)} \mathfrak{C}_{X}^{(i)} T$ for all vector fields $X \in p_{i}^{*}(T M)$.

In particular, we have for $i=1,2$

5. $S^{(i)}\left(\mathfrak{C}^{(i)} E^{(i)} T\right)=S^{(i)}\left(E^{(i)} \mathfrak{C}^{(i)} T\right)$, where $S^{(i)}$ is the symmetrization over the additional $p_{i}^{*}\left(T^{*} M\right)$-components arising from the derivation and the extension of $T$.

Notation Let $U_{1}, U_{2} \in S^{k}\left(p_{i}^{*}\left(T^{*} M\right)\right) \otimes \mathcal{F}_{r s k l}$ and $V \in S^{l}\left(p_{i}^{*}\left(T^{*} M\right)\right)(l \leq k)$ be symmetric. Then we note $U_{1} \equiv U_{2} \bmod V$ if there exist a symmetric kernel $W \in$ $S^{k-l}\left(T^{*} M\right) \otimes \mathcal{F}_{r s k l}$ such that $U_{1}-U_{2}=V \circ W$.

Proposition 6.10 $K^{(i)} T,(i=1,2)$ are kernels with simple transformation laws and satisfy

1. $\mathfrak{X}^{(i)} K^{(j)} T=K^{(j)} \mathfrak{X}^{(i)} T$ if $i \neq j$

2. $\left(\mathfrak{X}^{(i)} K^{(i)} T(P)\right)\left(\sigma_{1}\right)=K^{(i)}\left(\mathfrak{X}^{(i)} T(P)\left(\sigma_{1}\right)\right),(i=1,2)$ for all $\sigma_{1} \in p_{i}^{*}\left(T^{*} M\right)$ and any $P \in \mathcal{P}$

3. $\mathfrak{C}^{(i)} K^{(j)} T=K^{(j)} \mathfrak{C}^{(i)} T$ if $i \neq j$

4. $\mathfrak{C}_{X}^{(i)} K^{(i)} T=K^{(i)} \mathfrak{C}_{X}^{(i)} T$ for all vector fields $X \in p_{i}^{*}(T M)$.

In particular, we have for $i=1,2$

5. $S^{(i)}\left(K^{(i)} \mathfrak{C}^{(i)} T\right) \equiv S^{(i)}\left(\mathfrak{C}^{(i)} K^{(i)} T\right)-2 S^{(i)}\left(E^{(i)} C^{(i)} T\right) \bmod g^{(i)}$, where $S^{(i)}$ is the symmetrization over the additional $p_{i}^{*}\left(T^{*} M\right)$-components and $g^{(i)}=p_{i}^{*}(g) \in S^{2}\left(p_{i}^{*}\left(T^{*} M\right)\right)$. 
Similarly to Theorem 6.2 we have

Theorem 6.6 Let $T: \mathcal{P} \longrightarrow \Gamma\left(\mathcal{F}_{r s k l}\right)$ be a conformal gauge invariant kernel. Then the linear term of the symmetrized $k$-fold partial conformal gauge derivative $S^{(i)}\left(\mathfrak{C}^{(i) k} T\right)$ of $T$ satisfies

$$
\begin{aligned}
\mathfrak{X}^{(i)}\left(S^{(i)} \mathfrak{C}^{(i) k} T\right) \equiv & k(\omega-k+1) S\left(E \mathfrak{C}^{(i) k-1} T\right) \\
& +k S^{(i)}\left(\mathfrak{C}^{(i) k-1} K^{(i)} T\right) \quad \bmod g^{(i)}
\end{aligned}
$$

where $S^{(i)}$ is the symmetrization with respect to the $k$ additional $p_{i}^{*}\left(T^{*} M\right)$-components.

Remark An analogous statement of Theorem 6.3 is valid for the partial conformal gauge derivative $\mathfrak{C}^{(i)}$.

Now let $T \mathcal{P} \longrightarrow \Gamma\left(E^{*} \otimes E\right)$ be a conformal gauge invariant kernel. Denote by $\Delta$ the diagonal of $M^{n} \times M^{n}$, i.e. $\Delta=\left\{(m, m) \mid m \in M^{n}\right\} \subset M^{n} \times M^{n}$ and identify it with $M^{n}$. Restricting the mixed partial conformal gauge derivative of $T$ to $\Delta$ we obtain a mapping $\left.\mathfrak{C}^{(1) \alpha} \mathfrak{C}^{(2) \beta_{T}}\right|_{\Delta}: \mathcal{P} \longrightarrow \Gamma\left(\mathcal{E}_{\alpha+\beta 011}\right)$ with simple transformation law. Now we will symmetrize this mapping with respect to the $T^{*} M$-components and calculate the linear term of the arising map $\bmod g$.

Clearly, for a mapping $T: \mathcal{P} \longrightarrow \Gamma\left(\mathcal{F}_{r+\alpha s+\beta k l}\right)$ the symmetrizations $S^{(i)}$ with respect to the additional $p_{i}^{*}\left(T^{*} M\right)$-components and the symmetrization $S$ with respect to the additional $T^{*} M$-components satisfy

$$
S\left(\left.T\right|_{\Delta}\right)=S\left(\left.\left(S^{(1)} S^{(2)} T\right)\right|_{\Delta}\right)
$$

Therefore,

$$
\begin{aligned}
\mathfrak{X}\left(S\left(\left.\mathfrak{C}^{(1) \alpha} \mathfrak{C}^{(2) \beta} T\right|_{\Delta}\right)\right) & =\mathfrak{X} S\left(\left.S^{(1)} S^{(2)} \mathfrak{C}^{(1) \alpha} \mathfrak{C}^{(2) \beta} T\right|_{\Delta}\right) \\
& =S \mathfrak{X}\left(\left.S^{(1)} S^{(2)} \mathfrak{C}^{(1) \alpha} \mathfrak{C}^{(2) \beta} T\right|_{\Delta}\right) \\
& =S\left(\left.\mathfrak{X}^{(1)} S^{(1)} S^{(2)} \mathfrak{C}^{(1) \alpha} \mathfrak{C}^{(2) \beta} T\right|_{\Delta}+\left.\mathfrak{X}^{(2)} S^{(1)} S^{(2)} \mathfrak{C}^{(1) \alpha} \mathfrak{C}^{(2) \beta} T\right|_{\Delta}\right)
\end{aligned}
$$

On the other hand we know from the properties of the partial conformal gauge derivatives proven above

$$
\begin{aligned}
& \mathfrak{X}^{(1)} S^{(1)} S^{(2)} \mathfrak{C}^{(1) \alpha} \mathfrak{C}^{(2) \beta} T=S^{(2)} \mathfrak{C}^{(2) \beta} X^{(1)} S^{(1)} \mathfrak{C}^{(1) \alpha} T \\
& \quad=S^{(2)} \mathfrak{C}^{(2) \beta}\left(\alpha(\omega-\alpha+1) S^{(1)} E^{(1)} \mathfrak{C}^{(1) \alpha-1} T+g^{(1)} \circ T^{\prime}\right) \\
& \quad=\alpha(\omega-\alpha+1) S^{(1)} S^{(2)} E^{(1)} \mathfrak{C}^{(1) \alpha-1} \mathfrak{C}^{(2) \beta} T+g^{(1)} \circ T^{\prime \prime}
\end{aligned}
$$

and

$$
\begin{aligned}
& \mathfrak{X}^{(2)} S^{(1)} S^{(2)} \mathfrak{C}^{(1) \alpha} \mathfrak{C}^{(2) \beta} T=S^{(1)} \mathfrak{C}^{(1) \alpha} X^{(2)} S^{(2)} \mathfrak{C}^{(2) \beta} T \\
& \quad=\beta(\omega-\beta+1) S^{(1)} S^{(2)} E^{(2)} \mathfrak{C}^{(1) \alpha} \mathfrak{C}^{(2) \beta-1} T+g^{(2)} \circ T^{\prime \prime \prime}
\end{aligned}
$$

We conclude

$$
\begin{aligned}
\mathfrak{X}\left(S\left(\left.\mathfrak{C}^{(1) \alpha} \mathfrak{C}^{(2) \beta} T\right|_{\Delta}\right)\right) \equiv \quad & \alpha(\omega-\alpha+1) S\left(\left.E^{(1)} \mathfrak{C}^{(1) \alpha-1} \mathfrak{C}^{(2) \beta} T\right|_{\Delta}\right) \\
& +\beta(\omega-\beta+1) S\left(\left.E^{(2)} \mathfrak{C}^{(1) \alpha} \mathfrak{C}^{(2) \beta-1} T\right|_{\Delta}\right) \bmod g
\end{aligned}
$$


Now we will choose a suitable linear combination of mixed partial conformal gauge derivatives $\mathfrak{C}^{(1) \alpha} \mathfrak{C}^{(2) \beta} T$ such that the linear term of this combination vanishes $\bmod g$. Note that $\left.E^{(1)} \tilde{T}\right|_{\Delta}=\left.E^{(2)} \widetilde{T}\right|_{\Delta}$.

Theorem 6.7 Let $T: \mathcal{P} \longrightarrow \Gamma\left(E^{*} \otimes E\right)$ be a conformal gauge invariant kernel of weight $\omega \neq 0,1,2, \ldots$. We define for each integer $k \geq 0$

$$
\widehat{I}_{k} T:=\sum_{\alpha+\beta=k} c(\alpha, \beta) S\left(\left.\mathfrak{C}^{(1) \alpha} \mathfrak{C}^{(2) \beta} T\right|_{\Delta}\right)
$$

where $c(\alpha, \beta) \in \mathbb{R}$ are given by

$$
c(\alpha, \beta)=(-1)^{\alpha+\beta} \frac{\left(\begin{array}{c}
\omega \\
\alpha+\beta
\end{array}\right)\left(\begin{array}{c}
\alpha+\beta \\
\alpha
\end{array}\right)\left(\begin{array}{c}
\alpha+\beta-\omega-1 \\
\alpha
\end{array}\right)}{\left(\begin{array}{c}
\omega \\
\alpha
\end{array}\right)\left(\begin{array}{c}
2(\alpha+\beta)-2 \omega-2 \\
\alpha+\beta
\end{array}\right)} .
$$

Then the trace free part $I_{k}=\mathcal{T} \widehat{I}_{k}$ of $\widehat{I}_{k}$ is a conformal gauge invariant of weight $\omega$.

Proof. By Theorem 6.4 it suffices to prove $\mathfrak{X} \widehat{I}_{k} \equiv 0 \bmod g$. From (16) it follows that

$$
\begin{aligned}
\mathfrak{X} \widehat{I}_{k} T \equiv \sum_{\alpha+\beta=k}\left(c(\alpha, \beta) \alpha(\omega-\alpha+1) S\left(\left.E^{(1)} \mathfrak{C}^{(1) \alpha-1} \mathfrak{C}^{(2) \beta} T\right|_{\Delta}\right)\right. \\
\left.+c(\alpha, \beta) \beta(\omega-\beta+1) S\left(\left.E^{(2)} \mathfrak{C}^{(1) \alpha} \mathfrak{C}^{(2) \beta-1} T\right|_{\Delta}\right)\right) \\
\equiv \sum_{\alpha+\beta=k}\left(c(\alpha, \beta) \alpha(\omega-\alpha+1) S\left(\left.E^{(1)} \mathfrak{C}^{(1) \alpha-1} \mathfrak{C}^{(2) \beta} T\right|_{\Delta}\right)\right. \\
\left.\quad+c(\alpha-1, \beta+1)(\beta+1)(\omega-\beta) S\left(\left.E^{(1)} \mathfrak{C}^{(1) \alpha-1} \mathfrak{C}^{(2) \beta} T\right|_{\Delta}\right)\right) \bmod g
\end{aligned}
$$

The assertion now follows from

$$
c(\alpha, \beta) \alpha(\omega-\alpha+1)=-c(\alpha-1, \beta+1)(\beta+1)(\omega-\beta) .
$$

The maps $\widehat{I}_{k} T$ can also be given in the following form.

\section{Corollary 6.1}

$\widehat{I}_{k} T=\sum_{\alpha+\beta=k} b(\alpha, \beta) S \mathfrak{C}^{\alpha}\left(\left.\mathfrak{C}^{(2) \beta} T\right|_{\Delta}\right)$, where $b(\alpha, \beta)=(-1)^{\alpha} \frac{\left(\begin{array}{c}-2 \omega+\alpha+2 \beta-2 \\ \beta\end{array}\left(\begin{array}{c}\alpha+\beta-\omega-1 \\ \alpha\end{array}\right)\right.}{\left(\begin{array}{c}2(\alpha+\beta)-2 \omega-2 \\ \alpha+\beta\end{array}\right)}$

\section{Corollary 6.2}

$$
\widehat{I}_{k} T=\left.S\left(\mathfrak{C}^{(2) k} T\right)\right|_{\Delta}-\sum_{\alpha+\beta=k} d(\alpha, \beta) S \mathfrak{C}^{\alpha} \widehat{I}_{\beta} T, \text { where } d(\alpha, \beta)=\frac{\left(\begin{array}{c}
\alpha+\beta \\
\alpha
\end{array}\right)\left(\begin{array}{l}
\alpha+\beta-\omega-1 \\
\alpha+\beta
\end{array}\right)}{\left(\begin{array}{c}
\alpha+2 \beta-2 \omega-1 \\
\alpha
\end{array}\right)}
$$




\subsection{Construction of the moments}

In this subsection let $M^{n}$ be a manifold of even dimension $n, n \geq 4$ and $E$ and $\mathcal{P}$ as above. Consider a normally hyperbolic operator $P$ with associated Lorentzian metric $g$ and a conformal gauge transform $\widetilde{P}=P_{\varphi, A}$ of $P$ with associated metric $\widetilde{g}=e^{2 \varphi} g$. Recall that if we are given a geodesically normal domain $\Omega$ with respect to $g$ then there exist a geodesically normal domain $\widetilde{\Omega} \subset \Omega$ with respect to $\widetilde{g}$ and a domain $\Omega_{0} \subset \widetilde{\Omega} \subset \Omega$ which is causal with respect to $g$ and $\widetilde{g}$ and on this domain $\Omega_{0}$ the tail terms $T, \widetilde{T} \in \Gamma\left(\Omega_{0} \times \Omega_{0}, E^{*} \otimes E\right)$ of $P$ and $\widetilde{P}$ satisfy

$$
\widetilde{T}(x, y)=e^{-\frac{n-2}{2}(\varphi(x)+\varphi(y))} A^{-1} \cdot T(x, y)
$$

for all $x \in \Omega_{0}, y \in \mathcal{J}_{ \pm}^{\Omega_{0}}(x)$ (cf. Proposition 5.2). The aim of this section is to carry out the above described construction of conformal gauge invariants $I_{k}$ using the tail term $T$. Unfortunately, the tail term is not a conformal gauge invariant kernel, since its transformation law is only valid in a domain $\Omega_{0}$ depending on the operator $P$ and only if the second variable is chosen in the future or past cone of the first variable. We get round these difficulties in the following way. We divide $\mathcal{P}$ into classes of conformal gauge equivalent operators. Let $\mathcal{P}_{0}$ be one of this equivalence classes and $P_{0} \in \mathcal{P}_{0}$ one of its elements. Furthermore fix a causal domain $\Omega_{0}$ with respect to $P$. Let $T\left(P_{0}\right)$ be the tail term of $P_{0}$ defined on $\Omega_{0} \times \Omega_{0}$. Now we consider an arbitrary element $P$ of $\mathcal{P}_{0}$, i. e. $P=\left(P_{0}\right)_{\varphi, A}$ and set

$$
T(P)(x, y):=e^{-\frac{n-2}{2}(\varphi(x)+\varphi(y))} A^{-1} T\left(P_{0}\right)(x, y)
$$

for all $(x, y) \in \Omega_{0} \times \Omega_{0}$. Then

$$
T: \mathcal{P}_{0} \longrightarrow \Gamma\left(\Omega_{0} \times \Omega_{0}, E \otimes E^{*}\right)
$$

is a conformal gauge invariant. Now we can construct the mappings $\widehat{I}_{k}: \mathcal{P}_{0} \longrightarrow$ $\Gamma\left(\left.\left(T^{*} M\right)^{k} \otimes E^{*} \otimes E\right|_{\Omega_{0}}\right)$ with simple transformation law and its trace free parts $I_{k} T$ as above. The result is independent of the choice of $P_{0} \in \mathcal{P}_{0}$ since $I_{k} T$ depends only on the values of $T$ and $\mathfrak{C}^{(1) \alpha} \mathfrak{C}^{(2) \beta} T$ on the diagonal $\Delta\left(\Omega_{0} \times \Omega_{0}\right)$ and these are exactly the values of the original tail term and its partial conformal gauge derivatives. Hence, we obtain

Theorem 6.8 Let $M^{n}$ be a manifold of even dimension $n \geq 4$. We can assign to each normally hyperbolic operator $P: \Gamma(E) \longrightarrow \Gamma(E)$ a sequence $\left\{I_{k}(P)\right\}_{k \geq 0}$

$$
I_{k}(P) \in \Gamma\left(S^{k}\left(T^{*} M\right) \otimes E^{*} \otimes E\right)
$$

of symmetric trace free mappings that are conformal gauge invariants of weight $\omega=1-\frac{n}{2} . I_{k}(P)$ is called the moment of $P$ of order $k$.

Let $T(P)$ be the tail term of the operator $P$. Then the following formulas are valid for $I_{k}(P)$.

$$
\begin{aligned}
I_{k}(P)(x) & =\mathcal{T}\left(\sum_{\alpha+\beta=k} c(\alpha, \beta) S\left(\left.\mathfrak{C}^{(1) \alpha} \mathfrak{C}^{(2) \beta} T(P)\right|_{\Delta}\right)(x, x)\right) \\
& =\mathcal{T}\left(S\left(\left.\mathfrak{C}^{(2) k} T(P)\right|_{\Delta}(x, x)\right)-\sum_{\alpha+\beta=k} d(\alpha, \beta) S \mathfrak{C}^{\alpha} I_{\beta}(P)(x)\right)
\end{aligned}
$$


where $c(\alpha, \beta), d(\alpha, \beta) \in \mathbb{R}$ are given by

$$
\begin{aligned}
& c(\alpha, \beta)=(-1)^{\alpha+\beta} \frac{\left(\begin{array}{c}
1-\frac{n}{2} \\
\alpha+\beta
\end{array}\right)\left(\begin{array}{c}
\alpha+\beta \\
\alpha
\end{array}\right)\left(\begin{array}{c}
\alpha+\beta+\frac{n}{2}-2 \\
\alpha
\end{array}\right)}{\left(\begin{array}{c}
1-\frac{n}{2} \\
\alpha
\end{array}\right)\left(\begin{array}{c}
2(\alpha+\beta)+n-4 \\
\alpha+\beta
\end{array}\right)} \\
& d(\alpha, \beta)=\frac{\left(\begin{array}{c}
\alpha+\beta \\
\alpha
\end{array}\right)\left(\begin{array}{c}
\alpha+\beta+\frac{n}{2}-2 \\
\alpha+2
\end{array}\right)}{\left(\begin{array}{c}
\alpha+2 \beta+n-3 \\
\alpha
\end{array}\right)} .
\end{aligned}
$$

The next aim is to prove the equivalence of the property of a normally hyperbolic operator $P$ to be of Huygens type and the vanishing of its moments in the real analytic case. Let $\Omega \subset M^{n}$ be a causal domain such that $\left.E\right|_{\Omega}$ is a trivial bundle. Fix a trivialization of $\left.E\right|_{\Omega}$. Then partial derivatives of sections in $\mathcal{F}_{r s k l}$ are defined. Let $X$ be a vector in $T \Omega$ and $\Psi \in \Gamma\left(\left.\mathcal{F}_{r s k l}\right|_{\Omega \times \Omega}\right)$ a section in $\left.\mathcal{F}_{r s k l}\right|_{\Omega \times \Omega}$. Then $X^{(2)} \Psi$ denotes the derivative of $\Psi$ in direction $X^{(2)}=p_{2}^{*}(X) \in p_{2}^{*}(T \Omega) \subset T(\Omega \times \Omega)$.

Proposition 6.11 Let $P$ be a normally hyperbolic operator with tail term $T(P)$. If $I_{l}(P)=0$ on $M^{n}$ for $0 \leq l<k$ then

$$
I_{k}(P)(x)\left(X_{1}, \ldots, X_{k}\right)=X_{1}^{(2)} X_{2}^{(2)} \ldots X_{k}^{(2)} T(P)(x, x)
$$

for all $x \in \Omega$ and $X_{1}, \ldots, X_{k} \in T_{x} \Omega$.

Proof. For $k=0$ this is obvious since $I_{0}(P)(x)=T(P)(x, x)$. Now assume that the assertion is true for $0,1, \ldots, k-1$. Suppose $I_{l}(P)=0$ on $M^{n}$ for $0 \leq l<k$. Fix $x \in \Omega$. By our assumption $X_{1}^{(2)} X_{2}^{(2)} \ldots X_{l}^{(2)} T(P)(x, x)=0$ holds for $0 \leq l<k$ and all $X_{1}, \ldots, X_{l} \in \Gamma(T \Omega)$. Therefore

$$
\begin{array}{cc}
\nabla^{(2) l} T(P)\left(Y_{1}, \ldots, Y_{l}\right)(x, x)=0 & 0 \leq l<k \\
\nabla^{(2) k} T(P)\left(Y_{1}, \ldots, Y_{k}\right)(x, x)=Y_{1}^{(2)} Y_{2}^{(2)} \ldots Y_{k}^{(2)} T(P)(x, x) &
\end{array}
$$

for arbitrary $Y_{1}, \ldots, Y_{l} \in \Gamma(T \Omega)$ since all partial derivatives of order $<k$ vanish. We now obtain from Theorem 6.3 and the corresponding remark on partial conformal gauge derivatives

$$
\begin{aligned}
\mathfrak{C}^{(2) l} T(P)(x, x) & =0 \quad 0 \leq l<k \\
\mathfrak{C}^{(2) k} T(P)\left(Y_{1}, \ldots, Y_{k}\right)(x, x) & =\nabla^{(2) k} T(P)\left(Y_{1}, \ldots, Y_{k}\right)(x, x) \\
& =Y_{1}^{(2)} Y_{2}^{(2)} \ldots Y_{k}^{(2)} T(P)(x, x) \\
& =: \partial^{(2) k} T(P)\left(Y_{1}, \ldots, Y_{k}\right)(x, x)
\end{aligned}
$$

Note that $\partial^{(2) k} T(P)$ is in our case symmetric. By Theorem $6.8,3$.

$$
I_{k}(P)(x) \equiv \partial^{(2) k} T(P)(x, x) \bmod g .
$$

Now it suffices to prove that $\partial^{(2) k} T(P)(x, x)$ itself is trace free. Then the assertion will follow since the decomposition of $\partial^{(2) k} T(P)(x, x)$ into a trace free part and a symmetric product $g \circ T^{\prime}$ is unique. To calculate the trace of $\partial^{(2) k} T(P)(x, x)$ we show first

$$
P[T(P)(x, \cdot)](y)=0 \quad \text { if } y \in \mathcal{J}_{ \pm}^{\Omega}(x) .
$$


We know that in case $n \geq 4$ is even the fundamental solution has the following structure

$$
\left.G_{ \pm}^{\Omega}\right)(x)=\sum_{i=0}^{\frac{n-4}{2}} c_{(2, i)} U_{i}(x, \cdot) R_{ \pm}^{\Omega}(2+2 i, x)+c_{\left(2, \frac{n-2}{2}\right)} T(x, \cdot) R_{ \pm}^{\Omega}(n, x) .
$$

Hence, we have on the interior $\operatorname{int} \mathcal{J}_{ \pm}^{\Omega}(x)$ of $\mathcal{J}_{ \pm}^{\Omega}(x)$

$$
\left.P\left[G_{ \pm}^{\Omega}\right]\right|_{\operatorname{int} \mathcal{J}_{ \pm}^{\Omega}(x)}=\left.P\left[\sum \ldots+c_{\left(2, \frac{n-2}{2}\right)} T(x, \cdot) R_{ \pm}^{\Omega}(n, x)\right]\right|_{\operatorname{int} \mathcal{J}_{ \pm}^{\Omega}(x)} \cdot
$$

Consequently,

$$
0=\left.c_{\left(2, \frac{n-2}{2}\right)} P[T(x, \cdot)] \cdot R_{ \pm}^{\Omega}(n, x)\right|_{\text {int } J_{ \pm}^{\Omega}(x)}
$$

since all other summands are multiples of $R_{ \pm}^{\Omega}(2 m, x)$ with $2 m<n$ and have therefore supports in $\mathcal{C}_{ \pm}^{\Omega}(x)$ (cf. Proposition 2.4, 4. and 5.). On the other hand we know

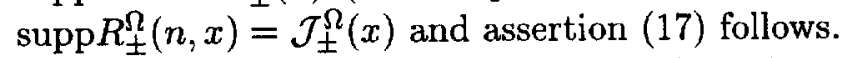

Now we differentiate equation $(17)(k-2)$ times and consider the result at $(x, x)$. Since all partial derivatives of $T$ of order less than $k$ vanish we obtain

$$
-\sum g^{i j} X_{1}^{(2)} X_{2}^{(2)} \ldots X_{k}^{(2)} \partial_{i}^{(2)} \partial_{j}^{(2)} T(P)(x, x)=0 .
$$

The l. h. s. of this equation is exactly the trace of $\partial^{(2) k} T(P)(x, x)$ and we conclude $I_{k}(P)(x)=\partial^{(2) k} T(P)(x, x)$.

Theorem 6.9 Let $M^{n}$ be a manifold of even dimension $n \geq 4$ and $P: \Gamma(E) \longrightarrow \Gamma(E)$ a normally hyperbolic operator.

1. If $P$ is a Huygens operator then all moments of $P$ vanish, i.e. $I_{k}(P) \equiv 0$ on $M^{n}$ for $k=0,1,2, \ldots$

2. If $M^{n}$ and $P$ are real analytic and $I_{k}(P) \equiv 0$ on $M^{n}$ for all $k=0,1,2, \ldots$ then $P$ is a Huygens operator.

\section{Proof.}

1. Fix a point $x \in M^{n}$ and a causal domain $\Omega$ containing $x$. If $P$ is of Huygens type then the tail term $T$ of $P$ satisfies $T(x, y)=0$ for all $y \in \mathcal{J}_{ \pm}^{\Omega}(x)$. Therefore all partial conformal gauge derivatives of $T$ vanish at $(x, x)$. Hence, $I_{k}(P) \equiv 0$ on $M^{n}$ for all $k=0,1,2, \ldots$ by definition.

2. If all $I_{k}(P)$ vanish then by Proposition $6.11 \partial_{i_{1}}^{(2)} \partial_{i_{2}}^{(2)} \ldots \partial_{i_{k}}^{(2)} T(P)(x, x)=0$ for all $k \geq 0$. Since $M^{n}$ and $P$ are analytic the tail term $T$ is analytic, too. A look at the Taylor expansion of $T$ near $(x, x)$ shows $T(P)(x, y)=0$. Consequently, $P$ is of Huygens type . 


\subsection{Moments of small order}

Now we are going to demonstrate how to calculate the moments $I_{k}(P)$ of a normally hyperbolic operator $P$. Since the moments are conformal gauge invariants it suffices to know $I_{k}\left(P_{\varphi}\right)$ for a special conformal transform $P_{\varphi}$ of $P$. Therefore we first try to choose the function $\varphi$ in such a way that the metric associated to $P_{\varphi}$ is especially suitable for computations.

\subsection{1 $x_{0}$ - adapted metrics}

Let $\left(M^{n}, g\right)$ be a pseudo-Riemannian manifold and let $x_{0} \in M^{n}$ be a fixed point. We choose a pseudo-orthonormal basis in the tangential space $T_{x_{0}} M$ and denote by $\left(g_{i j}\right)$ the coefficients of the metric $g$ in normal coordinates near $x_{0}$ with respect to this basis. We define functions $|g|$ and $\Delta(g)$ in a neighborhood of $x_{0}$ by

$$
|g|=\left|\operatorname{det}\left(g_{i j}\right)\right|, \quad \Delta(g)=\operatorname{det}\left(g_{i j}\right)
$$

Then $|g|$ and $\Delta(g)$ do not depend on the choice of the pseudo-orthonormal basis in $T_{x_{0}} M$.

Definition 6.15 Let $r \in \mathbb{N}$ be a fixed (large) positive integer. In the situation considered above the metric $g$ is said to be $x_{0}$ - adapted of order $r$ if the associated function $\Delta(g)$ satisfies

$$
\Delta(g)\left(\exp _{x_{0}} t U\right)=\Delta(g)\left(x_{0}\right)+O\left(t^{r}\right)
$$

for all $U \in T_{x_{0}} M$ and small $t<t_{0} \in \mathbb{R}$.

Our next aim is to prove that given a metric $g$ on $M^{n}, x_{0} \in M^{n}$ and $r \in \mathbb{N}$ we can find a conformal transformation such that the changed metric is $x_{0}$ - adapted. To begin with we discuss the Taylor formula for the coefficients of the metric $g$ in normal coordinates. First let us recall some facts on symmetric differentials.

Consider a $C^{\infty}$-function $f: \mathbb{R}^{n} \supset U(0) \longrightarrow \mathbb{R}$ on an open neighborhood $U(0)$ of $0 \in \mathbb{R}^{n}$. Define a symmetric tensor $d^{k} f(0) \in S^{k}\left(\mathbb{R}^{n}\right)^{*}$ by

$$
d^{k} f(0)(U, U, \ldots, U):=\left.\frac{d^{k}}{d r^{k}} f(r U)\right|_{r=0}
$$

for $U \in \mathbb{R}^{n}$. Then for $X_{1}, \ldots, X_{k} \in \mathbb{R}^{n}$

$$
d^{k} f(0)\left(X_{1}, \ldots, X_{k}\right)=X_{1} \ldots X_{k} f(0)
$$

holds where now $X_{1}, \ldots, X_{k}$ denote the "constant" vector fields $X_{i}(x)=X_{i}, i=$ $1, \ldots, k, x \in U(0)$. With this notation the Taylor formula for $f$ near $x_{0}$ has the form

$$
f(t U)=\sum_{k=0}^{N} \frac{t^{k}}{k !}\left(d^{k} f\right)(0)(U, U, \ldots, U)+O\left(t^{N+1}\right)
$$

for small $t \in \mathbb{R}$. 
Similarly, given a $C^{\infty}$-function $f: M^{n} \longrightarrow \mathbb{R}$ on a manifold $M^{n}$ we define

$$
\left(d^{k} f\right)\left(x_{0}\right)(U, U, \ldots, U):=\left.\frac{d^{k}}{d r^{k}} f\left(\exp _{x_{0}} r U\right)\right|_{r=0}
$$

for $x_{0} \in M^{n}, U \in T_{x_{0}} M$.

Now let $B \in \Gamma\left(\left(T^{*} M\right)^{p}\right)$ be a tensor field on $M^{n}$. Denote by $\nabla^{k} B \in \Gamma\left(\left(T^{*} M\right)^{p+k}\right)$ the $\mathrm{k}$-fold covariant derivative of $B$ with respect to the Levi-Civita connection defined by $g$. In particular we have

$$
\left(\nabla^{k} B\right)\left(x_{0}\right)(U, U, \ldots, U)=\left(\nabla_{U} \nabla_{U} \ldots \nabla_{U} B\right)\left(x_{0}\right)=:\left(\nabla_{U}^{k} B\right)\left(x_{0}\right)
$$

where now $U$ is the parallel vector field along $\exp _{x_{0}} t U$ with $U\left(x_{0}\right)=U$.

For a symmetric tensor field $B \in \Gamma\left(S^{p}\left(T^{*} M\right)\right)$ let the symmetric differential $d^{k} B \in$ $\Gamma\left(S^{p+k}\left(T^{*} M\right)\right)$ be the symmetrized k-fold covariant derivative, i.e.

$$
d^{k} B=S\left(\nabla^{k} B\right)
$$

If $X_{1}, \ldots, X_{k+p}$ are vector fields parallel along geodesics through $x_{0}$ then we obtain by polarization from (18) and (19)

$$
d^{k} B\left(X_{1}, \ldots, X_{k+p}\right)\left(x_{0}\right)=\frac{1}{(k+p) !} \sum_{\sigma \in S_{k+p}}\left(\nabla_{X_{\sigma(1)}} \ldots \nabla_{X_{\sigma(k)}} B\right)\left(X_{\sigma(k+1)}, \ldots, X_{\sigma(k+p)}\right) .
$$

The following properties of the symmetric differential can easily be proved.

1. If $f \in C^{\infty}\left(M^{n}\right), B \in \Gamma\left(S^{p}\left(T^{*} M\right)\right)$ then

$$
d^{k}(f \cdot B)=\sum_{i=0}^{k}\left(\begin{array}{c}
k \\
i
\end{array}\right) d^{i} f \circ d^{k-i} B
$$

2. If $A \in \Gamma\left(S^{p}\left(T^{*} M\right)\right), B \in \Gamma\left(S^{q}\left(T^{*} M\right)\right)$ then

$$
d(A \circ B)=d A \circ B+A \circ d B .
$$

3. If $B \in \Gamma\left(S^{p}\left(T^{*} M\right)\right)$ then

$$
\operatorname{Trace}_{g}(d B)=\frac{p-1}{p+1} d\left(\operatorname{Trace}_{g} B\right)-\frac{2}{p+1} \delta B
$$

where $\delta B:=-$ Trace $_{g} \nabla B$.

Now we turn to the Taylor formula for a pseudo-Riemannian metric in normal coordinates. We will see that the symmetric differentials of the metric coefficients are polynomials in covariant derivatives of the curvature tensor $R$. The following proof can be found in [BGM74]. Let $\left(M^{n}, g\right)$ be pseudo-Riemannian and $x_{0} \in M^{n}$. We 
fix a vector $U \in T_{x_{0}} M$. Choose $r_{0} \in \mathbb{R}, r_{0}>0$ such that $\exp _{x_{0}} r_{0} U$ exists and define $\gamma_{U}:\left[0, r_{0}\right] \longrightarrow M^{n}$ by $\gamma_{U}(r)=\exp _{x_{0}} r U$. Now we consider the map

$$
\begin{gathered}
\mathcal{Y}:\left[0, r_{0}\right] \longrightarrow T_{x_{0}} M^{n} \\
\mathcal{Y}(r)=\mathcal{P}\left(\gamma_{U}(r), x_{0}\right)\left(\operatorname{dexp}_{x_{0}}(r U)(Y)\right)
\end{gathered}
$$

where $\mathcal{P}\left(\gamma_{U}(r), x_{0}\right)$ denotes as usual the parallel displacement from $\gamma_{U}(r)$ to $x_{0}$ along $\gamma_{U}$. We verify that the coefficients in the Taylor formula of $\mathcal{Y}(r)$ near 0 are polynomials in the covariant derivatives of the curvature tensor. We use the fact that for all $Y \in$ $T_{x_{0}} M$ the vector $\operatorname{dexp}_{x_{0}}(r U)(Y)$ equals the value of that Jacobi field $\tilde{Y}_{r}$ along $\gamma_{U}$ in $\gamma_{U}(r)$ which satisfies $\widetilde{Y}_{r}\left(x_{0}\right)=0$ and $\nabla_{U} \widetilde{Y}_{r}\left(x_{0}\right)=\frac{Y}{r}$. Clearly $\widetilde{Y}_{r}=\frac{r_{0}}{r} \widetilde{Y}_{r_{0}}$ and therefore

$$
\mathcal{Y}(r)=\mathcal{P}\left(\gamma_{U}(r), x_{0}\right)\left(\widetilde{Y}_{r}\right)=\frac{r_{0}}{r} \mathcal{P}\left(\gamma_{U}(r), x_{0}\right)\left(\widetilde{Y}_{r_{0}}\right)
$$

Using the notation $\mathcal{Y}_{r_{0}}(r):=\mathcal{P}\left(\gamma_{U}(r), x_{0}\right)\left(\tilde{Y}_{r_{0}}\right)$ and taking into consideration that $\mathcal{Y}_{r_{0}}(0)=0$ we obtain

$$
\mathcal{Y}(r)=r_{0}\left(\mathcal{Y}_{r_{0}}^{\prime}(0)+\frac{r}{2 !} \mathcal{Y}_{r_{0}}^{\prime \prime}(0)+\ldots+\frac{r^{k}}{(k+1) !} \mathcal{Y}_{r_{0}}^{(k+1)}(0)+O\left(r^{k+1}\right)\right)
$$

On the other hand we have

$$
\mathcal{Y}_{r_{0}}^{(k)}(0)=\left.\frac{d^{k}}{d r^{k}} \mathcal{P}\left(\gamma_{U}(r), x_{0}\right)\left(\widetilde{Y}_{r_{0}}\right)\right|_{r=0}=\left(\nabla_{U}^{k} \widetilde{Y}_{r_{0}}\right)\left(x_{0}\right)
$$

Hence we have to calculate $\nabla_{\gamma_{U}^{\prime}}^{k} \widetilde{Y}_{r_{0}}$. Since $\widetilde{Y}_{r_{0}}$ is a Jacobi field $\nabla_{\gamma_{U}^{\prime}}^{2} \widetilde{Y}_{r_{0}}+\mathcal{R}\left(\gamma_{U}^{\prime}, \widetilde{Y}_{r_{0}}\right) \gamma_{U}^{\prime}$ $=0$ holds along $\gamma_{U}(r)$. Consequently,

$$
\nabla_{\gamma_{U}^{\prime}}^{k} \tilde{Y}_{r_{0}}=-\sum_{i=0}^{k-2}\left(\begin{array}{c}
k-2 \\
i
\end{array}\right)\left(\nabla_{\gamma_{U}^{\prime}}^{k-2-i} \mathcal{R}\right)\left(\gamma_{U}^{\prime}, \nabla_{\gamma_{U}^{\prime}}^{k} \tilde{Y}_{r_{0}}\right) \gamma_{U}^{\prime}, \quad k \geq 2 .
$$

Taking into consideration that by definition $\widetilde{Y}_{r}\left(x_{0}\right)=0$ and $\nabla_{U} \tilde{Y}_{r}\left(x_{0}\right)=\frac{Y}{r}$ we obtain

$$
\begin{aligned}
& \nabla_{U} \widetilde{Y}_{r}\left(x_{0}\right)=\frac{Y}{r} \\
& \nabla_{U}^{2} \tilde{Y}_{r}\left(x_{0}\right)=0 \\
& \nabla_{U}^{3} \widetilde{Y}_{r}\left(x_{0}\right)=-\frac{1}{r_{0}} \mathcal{R}(U, Y) U \\
& \nabla_{U}^{4} \widetilde{Y}_{r}\left(x_{0}\right)=-\frac{2}{r_{0}}(\nabla U \mathcal{R})(U, Y) U \\
& \nabla_{U}^{5} \tilde{Y}_{r}\left(x_{0}\right)=-\frac{3}{r_{0}}\left(\nabla_{U}^{2} \mathcal{R}\right)(U, Y) U+\frac{1}{r_{0}} \mathcal{R}(U, \mathcal{R}(U, Y) U) U \\
& \cdots \\
& \nabla_{U}^{k} \tilde{Y}_{r}\left(x_{0}\right)=-\frac{k-2}{r_{0}}\left(\nabla_{U}^{k-3} \mathcal{R}\right)(U, Y) U+\begin{array}{l}
\text { polynomials in covariant deri- } \\
\text { vatives } \nabla_{U}^{i} \mathcal{R} \text { of order } i \leq k-5
\end{array}
\end{aligned}
$$


We conclude

$$
\begin{aligned}
\mathcal{Y}(r)= & Y-\frac{r^{2}}{3 !} \mathcal{R}(U, Y) U-\frac{r^{3}}{4 !} \cdot 2 \cdot\left(\nabla_{U} \mathcal{R}\right)(U, Y) U \\
& -\frac{r^{4}}{5 !}\left(3\left(\nabla_{U}^{2} \mathcal{R}\right)(U, Y) U+\mathcal{R}(U, \mathcal{R}(U, Y) U) U\right) \\
& -\cdots \\
& -\frac{r^{k}}{(k+1) !}\left((k-1)\left(\nabla_{U}^{k-2} \mathcal{R}\right)(U, Y) U+\underset{\text { vatives } \nabla_{U}^{i} \mathcal{R} \text { of order } i \leq k-4}{\text { polynomials in covariant deri- }}\right) \\
& +O\left(r^{k+1}\right)
\end{aligned}
$$

Now we are able to write the Taylor formula for the coefficients $g_{i j}$ of the metric $\mathrm{g}$ with respect to normal coordinates centered in $x_{0}$. More generally, we consider $\left(\exp _{x_{0}}^{*}\right)(r U)(Y, Z)$, where $Y$ and $Z$ are the constant vector fields $Y(U) \equiv Y \in T_{x_{0}} M^{n}$, $Z(U) \equiv Z \in T_{x_{0}} M^{n}$ on $T_{x_{0}} M^{n}$. We have

$$
\begin{aligned}
& \left(\exp ^{*} g_{x_{0}}\right)_{r U}(Y, Z)=g_{\gamma_{U}(r)}\left(\operatorname{dexp}_{x_{0}}(r U)(Y), \operatorname{dexp}_{x_{0}}(r U)(Z)\right) \\
& \quad=g_{x_{0}}\left(\mathcal{P}\left(\gamma_{U}(r), x_{0}\right)\left(\operatorname{dexp}_{x_{0}}(r U)(Y)\right), \mathcal{P}\left(\gamma_{U}(r), x_{0}\right)\left(\exp _{x_{0}}(r U)(Z)\right)\right) \\
& \quad=g_{x_{0}}(\mathcal{Y}(r), \mathcal{Z}(r)) .
\end{aligned}
$$

Combining (26) and (27) we obtain

$$
\begin{aligned}
& \left(\exp ^{*} g\right)_{r U}(Y, Z)=g_{x_{0}}(Y, Z)+\frac{r^{2}}{2 !}\left(-\frac{2}{3} g_{x_{0}}(Y, \mathcal{R}(U, Z) U)\right) \\
& \quad+\frac{r^{3}}{3 !}\left(-\frac{2 \cdot 2}{4} g_{x_{0}}\left(Y,\left(\nabla_{U} \mathcal{R}\right)(U, Z) U\right)\right) \\
& \quad+\frac{r^{4}}{4 !}\left(-\frac{2 \cdot 3}{5} g_{x_{0}}\left(Y,\left(\nabla_{U}^{2} \mathcal{R}\right)(U, Z) U\right)+\frac{16}{15} g_{x_{0}}(\mathcal{R}(U, Y) U, \mathcal{R}(U, Z) U)\right) \\
& \quad+\ldots \\
& \quad+\frac{r^{k}}{k !}\left(-\frac{2(k-1)}{k+1} g_{x_{0}}\left(Y,\left(\nabla_{U}^{k-2} \mathcal{R}\right)(U, Z) U\right)+\begin{array}{l}
\text { polynomials in covariant deri- } \\
\text { vatives } \nabla_{U}^{i} \mathcal{R} \text { of order } i \leq k-4
\end{array}\right) \\
& \quad+O\left(r^{k+1}\right)
\end{aligned}
$$

This provides a rule for the calculation of

$$
d^{k}\left(\left(\exp ^{*} g\right)_{r U}(\cdot, \cdot)\right)\left(x_{0}\right):\left(T_{x_{0}} M\right)^{2} \longrightarrow S^{k}\left(T_{x_{0}}^{*} M\right)
$$

as polynomials in covariant derivatives of the curvature tensor $\mathcal{R}$.

We now associate to the metric $g$ on $M^{n}$ a sequence of symmetric tensors $N_{k}$ as follows. Consider the function $|g|: U\left(x_{0}\right) \longrightarrow \mathbb{R}$ as defined above on an open neighborhood of $x_{0} \in M^{n}$ and set

\section{Definition 6.16}

$$
N_{k}\left(x_{0}\right)=\frac{k+1}{2 k-2} d^{k} \ln |g|\left(x_{0}\right) \in S^{k}\left(T_{x_{0}}^{*} M\right), k=1,2, \ldots
$$


These tensors are related to the curvature tensor of $M^{n}$ in the following way.

Definition 6.17 For $A, B \in\left(T^{*} M\right)^{4}$ let $Q_{4}(A, B) \in S^{4}\left(T^{*} M\right)$ be the symmetric tensor

$$
Q_{4}(A, B):=S\left(\text { Trace }_{g(2,6)(4,8)} A \otimes B\right) .
$$

Proposition 6.12 The tensors $N_{k}$ defined as above satisfy

$$
\begin{aligned}
& N_{1}=0 \\
& N_{2}=\text { Ric } \\
& N_{3}=d \text { Ric } \\
& N_{4}=d^{2} \operatorname{Ric}-\frac{2}{9} Q_{4}(\mathcal{R}, \mathcal{R}) \\
& \ldots \\
& N_{k}=d^{k-2} \operatorname{Ric}+\begin{array}{l}
\text { polynomials in covariant deri- } \\
\text { vatives } \nabla_{U}^{i} \mathcal{R} \text { of order } i \leq k-3
\end{array}
\end{aligned}
$$

Proof. Let $s_{1}, \ldots, s_{n}$ be a pseudo-orthonormal basis in $T_{x_{0}} M$ and $\varepsilon_{\alpha}:=g\left(s_{\alpha}, s_{\alpha}\right)$. Denote by $g_{i j}$ the coefficients of $g$ in normal coordinates centered in $x_{0}$ with respect to this basis. From the Taylor formula of $g_{i j}$ we can get $d^{k}|g|\left(x_{0}\right)$. For this we use the Leibniz rule for the differentiation of the determinant. Let $\varepsilon$ be the number $\varepsilon=(-1)^{\text {index }(g)}$. Then $|g|=\varepsilon \cdot \Delta(g)$. In particular using the notation $R_{i j k l}=$ $=g_{x_{0}}\left(\mathcal{R}\left(s_{i}, s_{j}\right) s_{k}, s_{l}\right),\left(\nabla_{m} \mathcal{R}\right)_{i j k l}=g_{x_{0}}\left(\left(\nabla_{s_{m}} \mathcal{R}\right)\left(s_{i}, s_{j}\right) s_{k}, s_{l}\right), \operatorname{Ric}_{i j}=\operatorname{Ric}\left(s_{i}, s_{j}\right)\left(x_{0}\right)$ etc. we obtain

$$
\begin{aligned}
& \partial_{i}|g|\left(x_{0}\right)=0
\end{aligned}
$$

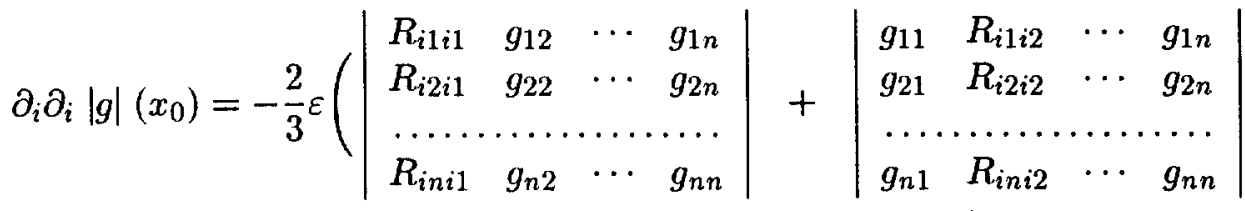

$$
\begin{aligned}
& \left.+\cdots+\left|\begin{array}{cccc}
R_{i 1 i 1} & g_{12} & \cdots & g_{1 n} \\
R_{i 2 i 1} & g_{22} & \cdots & g_{2 n} \\
\cdots \cdots & \cdots \cdots \cdots & \cdots \\
R_{i n i 1} & g_{n 2} & \cdots & g_{n n}
\end{array}\right|\right) \\
& =-\frac{2}{3} \varepsilon\left(\varepsilon \varepsilon_{1} R_{i 1 i 1}+\varepsilon \varepsilon_{2} R_{i 2 i 2}+\ldots+\varepsilon \varepsilon_{n} R_{i n i n}\right) \\
& =\frac{2}{3} \operatorname{Ric}_{i i}
\end{aligned}
$$

and similarly

$$
\begin{aligned}
\partial_{i} \partial_{i} \partial_{i}|g|\left(x_{0}\right) & =-\varepsilon\left(\varepsilon \varepsilon_{1}\left(\nabla_{1} \mathcal{R}\right)_{i 1 i 1}+\varepsilon \varepsilon_{2}\left(\nabla_{2} \mathcal{R}\right)_{i 2 i 2}+\ldots+\varepsilon \varepsilon_{n}\left(\nabla_{n} \mathcal{R}\right)_{i n i n}\right) \\
& =\left(\nabla_{i} \operatorname{Ric}\right)_{i i} \\
\nabla_{i}^{(4)}|g|\left(x_{0}\right) & =\frac{6}{5}\left(\nabla_{i}^{2} \operatorname{Ric}\right)_{i i}+\frac{16}{15} \sum_{\alpha, \beta} \varepsilon_{\alpha} \varepsilon_{\beta}\left(R_{i \alpha i \beta}\right)^{2}
\end{aligned}
$$




$$
\begin{gathered}
+\frac{4}{3} \sum_{\alpha \neq \beta} \varepsilon_{\alpha} \varepsilon_{\beta}\left(R_{i \alpha i \alpha} R_{i \beta i \beta}-\left(R_{i \alpha i \beta}\right)^{2}\right) \\
\nabla_{i}^{(k)}|g|\left(x_{0}\right)=c(k)\left(\nabla_{i}^{k-2} \operatorname{Ric}\right)_{i i}+\begin{array}{l}
\text { polynomials in covariant deri- } \\
\text { vatives } \nabla_{U}^{i} \mathcal{R} \text { of order } i \leq k-4
\end{array}
\end{gathered}
$$

Furthermore, we have

$$
\begin{aligned}
\partial_{i} \ln |g| & =\frac{1}{|g|} \partial_{i}|g| \\
\partial_{i} \partial_{i} \ln |g| & =-\frac{1}{|g|^{2}}\left(\partial_{i}|g|\right)^{2}+\frac{1}{|g|^{2}} \partial_{i} \partial_{i}|g| \\
\partial_{i} \partial_{i} \partial_{i} \ln |g| & =\frac{2}{|g|^{3}}\left(\partial_{i}|g|\right)^{3}-\frac{3}{|g|^{2}}\left(\partial_{i}|g|\right)\left(\partial_{i} \partial_{i}|g|\right)+f r a c 1|g| \partial_{i} \partial_{i} \partial_{i}|g| \\
\partial_{i}^{(4)} \ln |g| & =-\frac{6}{|g|^{4}}\left(\partial_{i}|g|\right)^{4}+\frac{12}{|g|^{3}}\left(\partial_{i}|g|\right)^{2}\left(\partial_{i} \partial_{i}|g|\right)-\frac{4}{|g|^{2}}\left(\partial_{i} \partial_{i} \partial_{i}|g|\right)\left(\partial_{i}|g|\right) \\
\ldots & -\frac{3}{|g|^{2}}\left(\partial_{i} \partial_{i}|g|\right)^{2}+\frac{1}{|g|} \partial_{i}^{(4)}|g| \\
\partial_{i}^{(k)} \ln |g| & =(-1)^{k}(k-1) ! \cdot \frac{1}{|g|^{k}}\left(\partial_{i}|g|\right)^{k}+\ldots+\frac{1}{|g|} \partial_{i}^{(k)}|g|
\end{aligned}
$$

and therefore

$$
\begin{aligned}
\partial_{i} \ln |g|\left(x_{0}\right)= & 0 \\
\partial_{i} \partial_{i} \ln |g|\left(x_{0}\right)= & \partial_{i} \partial_{i}|g|\left(x_{0}\right)=\frac{2}{3} \operatorname{Ric}_{i i} \\
\partial_{i} \partial_{i} \partial_{i} \ln |g|\left(x_{0}\right)= & \partial_{i} \partial_{i} \partial_{i}|g|\left(x_{0}\right)=\left(\nabla_{i} \operatorname{Ric}\right)_{i i} \\
\partial_{i}^{(4)} \ln |g|\left(x_{0}\right)= & -3\left(\partial_{i} \partial_{i}|g|\left(x_{0}\right)\right)^{2}+\partial_{i}^{(4)}|g|\left(x_{0}\right) \\
= & -\frac{4}{3}\left(\operatorname{Ric}_{i i}\right)^{2}+\frac{6}{5}\left(\nabla_{i}^{2} \operatorname{Ric}\right)_{i i}+ \\
& \frac{16}{15} \sum_{\alpha, \beta} \varepsilon_{\alpha} \varepsilon_{\beta}\left(R_{i \alpha i \beta}\right)^{2}+\frac{4}{3} \sum_{\alpha \neq \beta} \varepsilon_{\alpha} \varepsilon_{\beta}\left(R_{i \alpha i \alpha} R_{i \beta i \beta}-\left(R_{i \alpha i \beta}\right)^{2}\right) \\
= & -\frac{4}{3}\left(\sum_{\alpha \neq \beta} \varepsilon_{\alpha} \varepsilon_{\beta} R_{i \alpha i \alpha} R_{i \beta i \beta}+\sum_{\alpha}\left(R_{i \alpha i \alpha}\right)^{2}\right)+\frac{6}{5}\left(\nabla_{i}^{2} \operatorname{Ric}\right)_{i i}+ \\
& \frac{16}{15} \sum_{\alpha, \beta} \varepsilon_{\alpha} \varepsilon_{\beta}\left(R_{i \alpha i \beta}\right)^{2}+\frac{4}{3} \sum_{\alpha \neq \beta} \varepsilon_{\alpha} \varepsilon_{\beta}\left(R_{i \alpha i \alpha} R_{i \beta i \beta}-\left(R_{i \alpha i \beta}\right)^{2}\right) \\
= & \frac{6}{5}\left(\nabla_{i}^{2} \operatorname{Ric}\right)_{i i}+-\frac{4}{15} \sum_{\alpha, \beta} \varepsilon_{\alpha} \varepsilon_{\beta}\left(R_{i \alpha i \beta}\right)^{2} .
\end{aligned}
$$

Since $d^{k} \ln |g|\left(x_{0}\right)$ is symmetric we obtain

$$
\begin{aligned}
& d \ln |g|\left(x_{0}\right)=0 \\
& d^{2} \ln |g|\left(x_{0}\right)=\frac{2}{3} \operatorname{Ric}\left(x_{0}\right)
\end{aligned}
$$




$$
\begin{aligned}
d^{3} \ln |g|\left(x_{0}\right) & =d \operatorname{Ric}\left(x_{0}\right) \\
d^{4} \ln |g|\left(x_{0}\right) & =\frac{6}{5} d^{2} \operatorname{Ric}\left(x_{0}\right)-\frac{4}{15} Q_{4}(\mathcal{R}, \mathcal{R})\left(x_{0}\right) \\
\ldots & \\
d^{k} \ln |g|\left(x_{0}\right) & =c(k) d^{(k-2)} \operatorname{Ric}\left(x_{0}\right)+\begin{array}{l}
\text { polynomials in covariant deri- } \\
\text { vatives } \nabla_{U}^{i} \mathcal{R} \text { of order } i \leq k-4
\end{array}
\end{aligned}
$$

and the assertion follows.

Next we discuss the behaviour of the tensors $N_{k}$ under conformal transformation of the metric $g$. Let $\widetilde{g}$ be the changed metric $\widetilde{g}=e^{2 \varphi} g$. The Ricci tensors Ric ${ }^{g}$ and Ric ${ }^{\widetilde{g}}$ with respect to $g$ and $\tilde{g}$ satisfy

$$
\operatorname{Ric}^{\tilde{g}}=\operatorname{Ric}^{g}-(n-2) \nabla(d \varphi)-d \varphi \circ d \varphi+\left(\Delta^{g} \varphi-(n-2)|d \varphi|^{2}\right) g .
$$

By (29) and Proposition 6.12 we obtain for the tensors $N_{k}^{g}$ and $N_{k}^{\tilde{g}}$ associated to $g$ and $\tilde{g}$, respectively

$$
N_{k}^{\widetilde{g}}=N_{k}^{g}-(n-2) d^{k-2} \nabla^{g}(d \varphi)+d^{k-2}\left(\Delta^{g} \varphi \cdot g\right)+\begin{aligned}
& \text { terms containing deriva- } \\
& \text { tives of } \varphi \text { of order } \leq k-1
\end{aligned} .
$$

Since $\nabla(d \varphi)$ is symmetric we have $\nabla(d \varphi)=d^{2} \varphi$ and therefore $d^{k-2} \nabla^{g}(d \varphi)=d^{k} \varphi$. Consider now the third term. By (1) and because of $\nabla^{g} g=0$ we have $d^{k-2}\left(\Delta^{g} \varphi \cdot g\right)=$ $d^{k-2}\left(\Delta^{g} \varphi\right) \circ g$. We want to prove now that $d^{k-2}\left(\Delta^{g} \varphi\right)$ differs from - Trace $g\left(d^{k} \varphi\right)$ only by terms containing derivatives of $\varphi$ of order $\leq k-1$. Let $s_{1}, \ldots s_{n}$ be an orthonormal basis of $T_{x_{0}} M$. We extend $s_{1}, \ldots s_{n}$ to a local $f$ rame by parallel displacement along geodesics through $x_{0}$. Moreover let $X_{1}, \ldots, X_{k-2}$ be arbitrary vectors in $T_{x_{0}} M$ and extend them in the same way. Then

$$
\begin{aligned}
& \text { Trace }_{g}\left(d^{k} \varphi\right)\left(x_{0}\right)\left(X_{1}, \ldots, X_{k-2}\right)=\sum_{i=1}^{n} \frac{1}{k !}\left(2 \sum_{\sigma \in S_{k-2}} s_{i} s_{i} X_{\sigma(1)} \cdots X_{\sigma(k-2)} \varphi\right. \\
& \quad+2 \sum_{\sigma \in S_{k-2}} s_{i} X_{\sigma(1)} s_{i} X_{\sigma(2)} \cdots X_{\sigma(k-2)} \varphi+2 \sum_{\sigma \in S_{k-2}} s_{i} X_{\sigma(1)} X_{\sigma(2)} s_{i} X_{\sigma(3)} \cdots X_{\sigma(k-2)} \varphi \\
& \left.+\ldots+2 \sum_{\sigma \in S_{k-2}} X_{\sigma(1)} \cdots X_{\sigma(k-2)} s_{i} s_{i} \varphi\right)\left(x_{0}\right) \\
& \quad+\sum_{i=1}^{n} \frac{1}{(k-2) !} X_{\sigma(1)} \cdots X_{\sigma(k-2)} s_{i} s_{i} \varphi\left(x_{0}\right)+\begin{array}{l}
\text { terms containing deriva- } \\
\text { tives of } \varphi \text { of order } \leq k-1
\end{array}
\end{aligned}
$$

since $s_{i} X_{\sigma(j)}=X_{\sigma(j)} s_{i}+\left[s_{i}, X_{\sigma(j)}\right]$. Furthermore, this equals

$$
\begin{aligned}
= & \sum_{i=1}^{n} \frac{1}{(k-2) !} X_{\sigma(1)} \cdots X_{\sigma(k-2)}\left(\Delta^{g} \varphi+\text { derivatives of } \varphi \text { of order } 1\right)\left(x_{0}\right) \\
& +\begin{array}{l}
\text { terms containing deriva- } \\
\text { tives of } \varphi \text { of order } \leq k-1
\end{array} \\
= & d^{k-2}\left(\Delta^{g} \varphi\right)\left(x_{0}\right)\left(X_{1}, \ldots, X_{k-2}\right)+\begin{array}{l}
\text { terms containing deriva- } \\
\text { tives of } \varphi \text { of order } \leq k-1
\end{array}
\end{aligned}
$$


Finally,

$$
N_{k}^{\widetilde{g}}=N_{k}^{g}-(n-2) d^{k} \varphi-\operatorname{Trace}_{g}\left(d^{k} \varphi\right) \circ g+\begin{aligned}
& \text { terms containing deriva- } \\
& \text { tives of } \varphi \text { of order } \leq k-1
\end{aligned} .
$$

Now we are able to prove the following

Theorem 6.10 Let $\left(M^{n}, g\right)$ be a pseudo-Riemannian manifold, $x_{0} \in M^{n}$ and $r \in \mathbb{N}$ a (large) positive integer. Then there exists a function $\varphi \in C^{\infty}\left(M^{n}\right)$ such that $\tilde{g}=e^{2 \varphi} g$ is $x_{0}$-adapted of order $r$, i.e.

$$
\Delta(\tilde{g})\left(\exp _{x_{0}} t X\right)=\Delta(\tilde{g})\left(x_{0}\right)+O\left(t^{r}\right)
$$

for all $X \in T_{x_{0}} M^{n}$ and small $t>0$.

Proof. To begin with we prove the following assertion. Given a symmetric tensor $B \in S^{l}\left(T^{*} M^{n}\right)$ and positive real numbers $c_{1}, c_{2} \in \mathbb{R}$ there exists a further symmetric tensor $A \in S^{l}\left(T^{*} M^{n}\right)$ such that

$$
B=c_{1} A+c_{2}\left(\operatorname{Trace}_{g} A\right) \circ g .
$$

We verify this by induction. The assertion is obvious for $l=0,1$. Suppose it is proved for all $l<L$. Let $B \in S^{L}\left(T^{*} M^{n}\right)$ be fixed. Then $B$ admits a unique decomposition $B=\mathcal{T} B+B^{\prime} \circ g$ with $B^{\prime} \in S^{L-2}\left(T^{*} M^{n}\right)$. Set $n_{1}=\frac{(L-2)(L-3)}{L(L-1)}$ and $n_{2}=\frac{2(n+2 L-4)}{L(L-1)}$. By our assumption there is an $A^{\prime} \in S^{L-2}\left(T^{*} M^{n}\right)$ such that

$$
B^{\prime}=\left(c_{1}+c_{2} n_{2}\right) A^{\prime}+c_{2} n_{1} \text { Trace }_{g} A^{\prime} \circ g
$$

holds. Let $A \in S^{L}\left(T^{*} M^{n}\right)$ be the symmetric tensor $A=\frac{1}{c_{1}} \mathcal{T} B+A^{\prime} \circ g$. Then we obtain

$$
\begin{aligned}
c_{1} A+c_{2}\left(\operatorname{Trace}_{g} A\right) \circ g & =\mathcal{T} B+c_{1} A^{\prime} \circ g+c_{2} \operatorname{Trace}_{g}\left(A^{\prime} \circ g\right) \circ g \\
& \left.=\mathcal{T} B+c_{1} A^{\prime} \circ g+c_{2} n_{1}\left(\operatorname{Trace}_{g} A^{\prime}\right) \circ g\right) \circ g+c_{2} n_{2} A^{\prime} \circ g \\
& =\mathcal{T} B+B^{\prime} \circ g=B
\end{aligned}
$$

because of $\operatorname{Trace}_{g}\left(A^{\prime} \circ g\right)=n_{1} \operatorname{Trace}_{g} A^{\prime} \circ g+n_{2} A^{\prime}$ and the assertion is proved.

Now we apply this to the following problem. Given a metric $g$ on $M^{n}$, a point $x_{0} \in M^{n}$ and a positive integer $r \in \mathbb{N}$ find a function $\varphi \in C^{\infty}\left(M^{n}\right)$ such that

$$
\varphi\left(x_{0}\right)=1, d \varphi\left(x_{0}\right)=0, N_{2}^{\tilde{g}}\left(x_{0}\right)=0, \ldots, N_{k}^{\tilde{g}}\left(x_{0}\right)=0, \ldots, N_{r}^{\tilde{g}}\left(x_{0}\right)=0
$$

where $\widetilde{g}=e^{2 \varphi} g$. A look at equation (31) shows that we have to solve the system

$$
(n-2) d^{k} \varphi\left(x_{0}\right)+\operatorname{Trace}_{g}\left(d^{k} \varphi\right) \circ g_{x_{0}}=N_{k}^{g}\left(x_{0}\right)+r_{k-1}, 1<k<r
$$

where $r_{k-1}$ contains only derivatives of $\varphi$ of order less than $k$. This can be done by induction. The term $r_{k-1}$ is known from the previous steps and the assertion proven above gives us a solution of (32). Hence, there is a function $\varphi \in C^{\infty}\left(M^{n}\right)$ such that $N_{k}^{\tilde{g}}\left(x_{0}\right)=0,0<k<r$ for $\tilde{g}=e^{2 \varphi} g$. 
In particular, $d^{k} \ln |\tilde{g}|\left(x_{0}\right), 0<k<r$ holds. Since on the other hand

$$
\begin{aligned}
d|\widetilde{g}|\left(x_{0}\right) & =d \ln (|\widetilde{g}|)\left(x_{0}\right) \\
d^{k}|\widetilde{g}|\left(x_{0}\right) & =d^{k} \ln |\widetilde{g}|\left(x_{0}\right)+\text { terms in } d^{s}|\widetilde{g}|\left(x_{0}\right), s<k
\end{aligned}
$$

it turns out that $d^{k}|\widetilde{g}|\left(x_{0}\right)=0$ for $0<k<r$. Because of $\Delta(\widetilde{g})=\varepsilon|\widetilde{g}|$ the assertion of the theorem now follows from the Taylor formula for $|\widetilde{g}|$.

Corollary 6.3 If the metric $g$ is $x_{0}$-adapted then the curvature tensor $\mathcal{R}$, the Ricci tensor Ric, the scalar curvature $R$, and the Schouten tensor $L$ associated to $g$ satisfy at $x_{0}$

$$
\begin{gathered}
\operatorname{Ric}\left(x_{0}\right)=0, \quad d \operatorname{Ric}\left(x_{0}\right)=0, \quad d^{2} \operatorname{Ric}\left(x_{0}\right)=\frac{2}{9} Q_{4}(\mathcal{R}, \mathcal{R}) \\
R\left(x_{0}\right)=0, \quad d R\left(x_{0}\right)=0 \\
L\left(x_{0}\right)=0, \quad d L\left(x_{0}\right)=0, \quad d^{2} L\left(x_{0}\right) \equiv-\frac{2}{9(n-2)} Q_{4}(\mathcal{R}, \mathcal{R}) \bmod g_{x_{0}}
\end{gathered}
$$

Proof. The first three equations follow from the proof of Theorem 6.10. It remains to show $d R\left(x_{0}\right)=0$. On one hand $\delta$ Ric $=-\frac{1}{2} d R$ holds on every (pseudo-) Riemannian manifold. On the other hand $d \operatorname{Ric}\left(x_{0}\right)=0$ implies with the same notation as above $\left(\left(\nabla_{k} \text { Ric }\right)_{i i}+2\left(\nabla_{i} \text { Ric }\right)_{i k}\right)\left(x_{0}\right)=0$. Hence, at $x_{0}$

$$
(\delta \mathrm{Ric})_{k}=-\sum_{i} \varepsilon_{i}\left(\nabla_{i} \mathrm{Ric}\right)_{i k}=\sum_{i} \varepsilon_{i} \frac{1}{2}\left(\nabla_{k} \mathrm{Ric}\right)_{i i}=\frac{1}{2} d R\left(s_{k}\right)
$$

and the assertion follows.

\subsubsection{The moments of small order for $x$-adapted metrics}

Now, we can express the first five moments $I_{j}(x), j=0, \ldots, 4$, of a normally hyperbolic operator $P$ for $x$-adapted metrics. Let $B \in \Gamma\left(S^{l}\left(T^{*} M\right) \otimes E\right)$ be a smooth symmetric tensor with values in $E$. The $k$-fold symmetric differential of $B$ is defined by $d^{k} B:=$ $S\left(\left(\nabla^{P}\right)^{k} B\right)$ where $\nabla^{P}$ is the derivative in $\Gamma\left(S^{l}\left(T^{*} M\right) \otimes E\right)$ defined by the Lorentzian metric and the covariant derivative $\nabla^{P}$ in $E$ defined by $P$ via its Weitzenböck formula. Let $U \in \Gamma\left(E^{*} \otimes E\right)$. In order to simplify the notation we denote by $d^{k} U(x, x)$ the $k$-fold symmetric differential of $U(x, \cdot)$ in the second component of the product $M \times M$ evaluated at $x: d^{k} U(x, x):=d^{k} U(x, \cdot)(x)$.

Proposition 6.13 Let $\left(M^{n}, g\right)$ be a Lorentzian manifold of even dimension $n \geq 4$ with a metric adapted to the point $x \in M$. Then the moments $I_{0}(x), \ldots, I_{4}(x)$ of a normally hyperbolic operator $P$ in the point $x$ satisfy the following recursion formula

$$
I_{k}(x)=\mathcal{T}\left(d^{k} U_{\frac{n-2}{2}}(x, x)-\sum_{l=1}^{k} d(k, l) d^{l} I_{k-l}(x)\right)+J_{k}
$$

where $U_{\frac{n-2}{2}}$ is the Hadamard coefficient of $P$,

$$
J_{k}= \begin{cases}0 & 0 \leq k \leq 3 \\ \frac{1}{9}(2 d(4,4)-1) \mathcal{T}\left(Q_{4}(\mathcal{R}, \mathcal{R})_{x}\right) I_{0}(x) & k=4\end{cases}
$$


and

$$
d(k, l)=\left(\begin{array}{l}
k \\
l
\end{array}\right)\left(\begin{array}{c}
k+\frac{n}{2}-2 \\
l
\end{array}\right)\left(\begin{array}{c}
2 k-l+n-3 \\
l
\end{array}\right)^{-1} .
$$

Proof: Since $g$ is adapted to $x \in M$ the Schouten tensor $L$ satisfies

$$
L_{x}=0, \quad d L_{x}=0, \quad d^{2} L_{x} \equiv-\frac{2}{9(n-2)} Q_{4}(\mathcal{R}, \mathcal{R})_{x} \bmod g_{x}
$$

Let $T \in \Gamma\left(\Omega_{0} \times \Omega_{0}, E^{*} \otimes E\right)$ be the tail term of $P$ on a causal neighbourhood of $x$. According to Theorem 6.8 the moments are given by

$$
I_{k}(x)=\mathcal{T}\left(S \mathfrak{C}^{k} T(x, x)-\sum_{l=1}^{k} d(k, l) S \mathfrak{C}^{l} I_{k-l}(x)\right)
$$

Here all derivatives refer to the second component of the product $M \times M$. If an equality or an equivalence mod $g$ holds only in the point $x$ or $(x, x)$ we denote this by $\stackrel{x}{=}$ and $\stackrel{x}{=}$, respectively.

We first prove the following relations between the conformal gauge derivatives and the symmetric differentials of a conformal gauge invariant $B: \mathcal{P} \longrightarrow \Gamma\left(S^{p}\left(T^{*} M\right) \otimes\right.$ $H o m(E, E))$ of weight $\omega$ and order $p \leq 4-k$

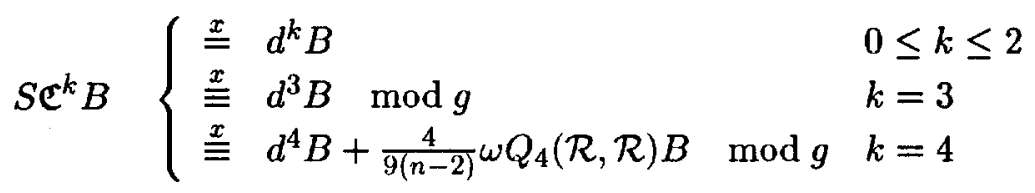

The linear term $\mathfrak{X} B$ of a conformal gauge invariant $B$ vanishes, hence $\mathfrak{C} B=\nabla B$ and $S \mathfrak{C} B=d B$. According to Theorem 6.1 the linear term of $\mathfrak{C} B$ is given by

$$
\begin{aligned}
\mathfrak{X C} B & =\mathfrak{C X} B+K B+2 \omega E B \\
& =K B+2 \omega E B .
\end{aligned}
$$

Hence,

$$
\begin{aligned}
\mathfrak{C}^{2} B & \left.=\nabla^{2} B-L\right\lrcorner(K B+2 \omega E B) \\
& \left.=\nabla^{2} B-L\right\lrcorner K B-2 \omega L \otimes B
\end{aligned}
$$

Because of $L_{x}=0$ this gives $S \mathfrak{C}^{2} B \stackrel{x}{=} d^{2} B$. Furthermore, from (36) follows

$$
\begin{aligned}
\mathfrak{C}^{3} B & \left.=\nabla \mathfrak{C}^{2} B-L\right\lrcorner \mathfrak{X} \mathfrak{C}^{2} B \\
& \left.\left.=\nabla^{3} B-2 \omega(\nabla L \otimes B+L \otimes \nabla B)-\nabla(L\lrcorner K B\right)-L\right\lrcorner \mathfrak{X C}^{2} B .
\end{aligned}
$$

Since $L_{x}=0$ and $d L_{x}=0$ follows $\left.S C^{3} B \stackrel{x}{=} d^{3} B-S(\nabla L\lrcorner K B\right)$. In case $p=0$ we have $K B=0$. Therefore, $S \mathfrak{C}^{3} B \stackrel{x}{=} d^{3} B$. Using the definition of the operation $K$ we get in case $p=1$ for a vector $U \in T_{x} M$

$$
S(\nabla L\lrcorner K B)(U, U, U, U)=2\left(\nabla_{U} L\right)(U, U) B(U)-g(U, U) B\left(\left(\nabla_{U} L\right)^{\sharp}\right)
$$


Hence,

$$
S(\nabla L\lrcorner K B)=2 d L \circ B-g B_{1} \stackrel{x}{=} 0 \bmod g
$$

and therefore, $S \mathfrak{C}^{3} B \stackrel{x}{=} d^{3} B \bmod g$. From (37) follows for an invariant $B$ of order $p=0$

$$
\left.\nabla \mathfrak{C}^{3} B=\nabla^{4} B-2 \omega\left(\nabla^{2} L \otimes B+2 \nabla L \otimes \nabla B+L \otimes \nabla^{2} B\right)-\nabla(L\lrcorner \mathfrak{X} \mathfrak{C}^{2} B\right)
$$

Then

$$
S \mathfrak{C}^{4} B \stackrel{x}{=} S \nabla \mathfrak{C}^{3} B \stackrel{x}{=} d^{4} B-2 \omega d^{2} L \circ B-S \nabla\left(L-S \mathfrak{X} \mathfrak{C}^{2} B\right)
$$

Using Theorem 6.1, Proposition 6.5 and (35) we obtain

$$
\begin{aligned}
S \mathfrak{X C} \mathfrak{C}^{2} B & =S \mathfrak{C X C} B+S K \mathfrak{C} B+2 \omega S E \mathfrak{C} B \\
& =2 \omega S \mathfrak{C E} B+2 \omega S E \mathfrak{C} B+S K \mathfrak{C} B \\
& =4 \omega S E \mathfrak{C} B+S K \mathfrak{C} B
\end{aligned}
$$

Regarding $L_{x}=0$ and $d L_{x}=0$ it follows

$$
\left.\left.\left.S \nabla(L\lrcorner S \mathfrak{X} \mathfrak{C}^{2} B\right) \stackrel{x}{=} 4 \omega S(\nabla L \otimes \nabla B)+S(\nabla L\lrcorner K \nabla B\right) \stackrel{x}{=} S(\nabla L\lrcorner K \nabla B\right)
$$

In the same way as in (38) we obtain $S(\nabla L\lrcorner K \nabla B) \stackrel{x}{=} 0 \bmod g$. Therefore, $\left.\left.S \nabla(L\lrcorner S \mathfrak{X C} \mathfrak{C}^{2} B\right)\right) \stackrel{x}{=} 0 \bmod g$ and finally

$$
\begin{aligned}
S \mathfrak{C}^{4} B & \stackrel{x}{=} d^{4} B-2 \omega d^{2} L \circ B \\
& \stackrel{x}{=} d^{4} B+\frac{4}{9(n-2)} \omega Q_{4}(\mathcal{R}, \mathcal{R}) B \bmod g
\end{aligned}
$$

The moments are conformal gauge invariants of weight $\omega=-\frac{n-2}{2}$. Therefore we can apply formula (34) with $\omega=-\frac{n-2}{2}$. For the tail term and the partial conformal gauge derivative we can use (34) replacing $2 \omega$ by $\omega=-\frac{n-2}{2}$. Then from (33) we obtain for the moments the formula

$$
I_{k}(x)=\mathcal{T}\left(d^{k} T(x, x)-\sum_{l=1}^{k} d(k, l) d^{l} I_{k-l}(x)\right)+J_{k} .
$$

According to Theorem 3.1 the tail term has the asymptotic expansion

$$
T(x, y) \underset{(x, y) \rightarrow C}{\widetilde{\frac{n-2}{2}}}(x, y)+\sigma(x, y) \tilde{T}(x, y)
$$

where $\tilde{T} \in \Gamma\left(\Omega_{0} \times \Omega_{0}, E^{*} \otimes E\right)$ is a smooth section and $\sigma$ is the quadratic geodesic distance function. Therefore, in $x$

$$
d^{k} T \stackrel{x}{=} d^{k} U_{\frac{n-2}{2}}+d^{k}(\sigma \tilde{T})=d^{k} U_{\frac{n-2}{2}}+\sum_{i=0}^{k}\left(\begin{array}{c}
k \\
i
\end{array}\right) d^{i} \sigma \circ d^{k-i} \tilde{T}
$$


holds. The quadratic geodesic distance function satisfies

$$
d^{i} \sigma(x, x)= \begin{cases}0 & i \neq 2 \\ 2 g_{x} & i=2\end{cases}
$$

Hence, $\quad d^{k} T \stackrel{x}{=} d^{k} U_{\frac{n-2}{2}} \quad \bmod g$. This proves the proposition.

We want to calculate the first moments for normally hyperbolic operators on 4dimensional Lorentzian manifolds. According to Proposition 6.13 we need the symmetric differentials of the first Hadamard coefficient $U_{1}$ of the operator.

Proposition 6.14 Let $P: \Gamma(E) \longrightarrow \Gamma(E)$ be a normally hyperbolic operator acting on a bundle $E$ over a 4-dimensional Lorentzian manifold $\left(M^{4}, g\right)$ with $x$-adapted metric $g$ of order $r \in \mathbb{N}$. Then the symmetric differentials of the first Hadamard coefficient $U_{1}$ of $P$ satisfy

$$
2(l+1) d^{l} U_{1}(x, x)=-d^{l} P \mathcal{P}(x, x) \quad l \leq r-3,
$$

where $\mathcal{P}(x, y) \in \Gamma\left(\operatorname{Hom}\left(E_{x}, E_{y}\right)\right)=\Gamma\left(E_{x}^{*} \otimes E_{y}\right)$ is the parallel displacement from $E_{x}$ to $E_{y}$ along geodesics with respect to $\nabla^{P}$ and all derivatives refer to the second component of the product $M \times M$.

We use Proposition 3.2 to calculate the Hadamard coefficient $U_{1}(x, y)$ of $P$ in normal coordinates. Let $\varphi(y)=\left(y_{1}, \ldots, y_{n}\right)$ be normal coordinates in a neighbourhood $\Omega$ of the point $x \in M$, arising from an orthonormal basis in $T_{x} M$. Then $\varphi(x)=0$ and $\left|\operatorname{det} g_{i j}(0)\right|=1$. In normal coordinates the quadratic geodesic distance function is given by

$$
\sigma(x, y)=\sum_{i j} g_{i j}\left(y_{1}, \ldots, y_{n}\right) y_{i} y_{j}=\sum_{i j} g_{i j}(0) y_{i} y_{j}
$$

Using $\sum_{k} g_{k j}(0) y_{k}=\sum_{k} g_{k j}(y) y_{k}$ for the divergence measure results

$$
m(x, \cdot)=-\frac{1}{2} \Delta \sigma(x, \cdot)-4=\frac{1}{2} \mathbf{r}_{x}\left(\ln \left|\operatorname{det}\left(g_{i j}\right)\right|\right)
$$

where $\mathbf{r}_{x}=\sum_{j} y_{j} \frac{\partial}{\partial y_{j}}$ is the radial vector field with respect to $x \in \Omega$. Let $\gamma(s)$ be the geodesic in $\Omega$ joining $x$ and $y$. Then because of $\mathbf{r}_{x}(\gamma(s))=s \cdot \gamma^{\prime}(s)$ follows

$$
\begin{aligned}
\tau(x, y) & :=\exp \left\{\frac{1}{2} \int_{0}^{1} \frac{m(x, \gamma(s))}{s} d s\right\}=\exp \left\{\frac{1}{4} \int_{0}^{1} \frac{\mathbf{r}_{x}(\ln |\operatorname{det}(g)|)}{s} d s\right\} \\
& =\exp \left\{\frac{1}{4} \int_{0}^{1} \frac{d}{d s}(\ln |\operatorname{det} g(\gamma(s))|) d s\right\}=\left|\frac{\operatorname{det} g(y)}{\operatorname{det} g(0)}\right|^{\frac{1}{4}}=|\operatorname{det} g(y)|^{\frac{1}{4}}
\end{aligned}
$$

Since $g$ is $x$-adapted of order $r$ we know that $|\operatorname{det} g(y)|=1+O\left(|\varphi(y)|^{r}\right)$. Hence $\frac{1}{\tau(x, y)}=1+O\left(|\varphi(y)|^{r}\right)$. According to Proposition 3.2 the 0 . Hadamard coefficient 
$U_{0}$ of $P$ is given by $U_{0}(x, y)=\left(1+O\left(|\varphi(y)|^{r}\right)\right) \mathcal{P}(x, y)$, where $\mathcal{P}(x, y) \in E_{x}^{*} \otimes E_{y}=$ $\operatorname{Hom}\left(E_{x}, E_{y}\right)$ is the parallel displacement from $E_{x}$ to $E_{y}$ along $\gamma$ with respect to the covariant derivative $\nabla^{P}$ defined by $P$. Furthermore, since $P$ is of second order $U_{1}(x, \cdot)$ satisfies the differential equation

$$
\nabla_{\mathbf{r}_{x}}^{P} U_{1}(x, \cdot)+\left(\frac{1}{2} m(x, \cdot)+1\right) U_{1}(x, \cdot)=-\frac{1}{2} P U_{0}(x, \cdot)=-\frac{1}{2} P \mathcal{P}(x, \cdot)+O\left(|\varphi(\cdot)|^{r-2}\right)
$$

Using (39) we obtain $m(x, y)=O\left(|\varphi(y)|^{r}\right)$. Hence,

$$
\left(\nabla_{\mathbf{r}_{x}}^{P}+1\right) U_{1}(x, \cdot)=-\frac{1}{2} P \mathcal{P}(x, \cdot)+O\left(|\varphi(\cdot)|^{r-2}\right) .
$$

Let $\left(u_{1}, \ldots, u_{m}\right)$ be a basis in the bundle $\left.E\right|_{\Omega}$ arising from a basis in the fibre $E_{x}$ by parallel displacement along geodesics through $x$. Then $\mathcal{P}(x, y)=\sum_{J=1}^{m} u_{J}^{*}(x) \otimes u_{J}(y)$. Let $P u_{I}=\sum_{J} B_{I J} u_{J}$ and $U_{1}(x, y)=\sum_{I J} U_{1}(x, y)_{I J} u_{I}^{*}(x) \otimes u_{J}(y)$. Then from (40) follows

$$
\left(1+y_{i} \frac{\partial}{\partial y_{i}}\right) U_{1}(x, \cdot)_{I J}=-\frac{1}{2} B_{I J}+O\left(|\varphi(\cdot)|^{r-2}\right) \quad I, J=1, \ldots, m
$$

If we insert in (41) the Taylor expansion of $U_{1}(x, \cdot)_{I J}$ and $B_{I J}$ with respect to the normal coordinates around $\varphi(x)=0$ and compare the coefficients up to the order $r-3$ we obtain

$$
\sum_{\pi \in S_{l}}\left(\partial_{j_{\pi(1)}} \ldots \partial_{j_{\pi(l)}} B_{I J}\right)(x)=-2(l+1) \sum_{\pi \in S_{l}}\left(\partial_{j_{\pi(1)}} \ldots \partial_{j_{\pi(l)}} U_{1}(x, \cdot)\right)(x) \quad l \leq r-3,
$$

where $\partial_{k}$ denotes the derivative with respect to the canonic basis vector $\frac{\partial}{\partial y_{k}}$. By definition of the symmetric differential $d^{l}=S\left(\nabla^{P}\right)^{l}$ this gives

$$
2(l+1) d^{l} U_{1}(x, x)=-d^{l} P \mathcal{P}(x, x) \quad l \leq r-3
$$

where the differentiation refer to the second component of the product $M \times M$.

\subsubsection{The moment of order 0}

We are going now to calculate the moment $I_{0}(P)$ for a given normally hyperbolic operator $P$ on $E$ over $M^{4}$ with associated Lorentzian metric $g$. Let $x \in M^{4}$ be fixed. We transform $g$ conformally to obtain an $x$-adapted metric $\tilde{g}=e^{2 \varphi} g$ of sufficiently high order. The following calculations are carried out with respect to this metric and the transformed operator $\widetilde{P}=P_{\varphi}$. From Proposition 6.13 we know

$$
I_{0}(P)(x)=\mathcal{T}\left(U_{1}(x, x)\right)=U_{1}(x, x)
$$


and from Proposition 6.14

$$
U_{1}(x, x)=-\frac{1}{2} P \mathcal{P}(x, x)
$$

Furthermore we have for $\widetilde{P}$ the Weitzenböck formula

$$
\widetilde{P}=\Delta^{\nabla^{\widetilde{P}}}+H_{\widetilde{P}}
$$

Let $u_{1}, \ldots, u_{m}$ be a local basis in $E$ such that $u_{I} 1 \leq I \leq m$ is parallel with respect to $\nabla^{\widetilde{P}}$ along geodesics through $x$. Furthermore, let $s_{1}, \ldots, s_{4}$ be a local pseudo-orthonormal frame in $T\left(M^{4}\right)$ parallel along geodesics through $x$. In particular $\nabla_{s_{i}} s_{i}(x)=0$ and $\nabla_{s_{i}}^{\widetilde{P}} \nabla_{s_{i}}^{\widetilde{P}} u_{I}(x)=0$ hold. Then we have

$$
\widetilde{P} \mathcal{P}(x, x)=\sum_{I=1}^{m} u_{I}^{*}(x) \otimes\left(\widetilde{P} u_{I}\right)(x)=\sum_{I=1}^{m} u_{I}^{*}(x) \otimes\left(\Delta^{\nabla^{\widetilde{P}}}+H_{\widetilde{P}}\right)(x) .
$$

Since $\Delta \nabla^{\widetilde{P}} u_{I}(x)=-\sum_{i=1}^{4} \varepsilon_{i}\left(\nabla_{s_{i}}^{\widetilde{P}} \nabla_{s_{i}}^{\widetilde{P}} u_{I}+\nabla_{\nabla_{s_{i}} s_{i}}^{\widetilde{P}} u_{I}\right)(x)=0$ where as usual $\varepsilon_{i}=\widetilde{g}\left(s_{i}, s_{i}\right)$ we conclude

$$
\tilde{P} \mathcal{P}(x, x)=\sum u_{I}^{*}(x) \otimes\left(H_{\widetilde{P}} u_{I}\right)(x)=H_{\widetilde{P}}(x) \in E_{x}^{*} \otimes E_{x} \cong \operatorname{Hom}\left(E_{x}, E_{x}\right) .
$$

On the other hand we observed that the scalar curvature $R(x)$ of the $x$-adapted metric vanishes at $x$. Hence, $H_{\widetilde{P}}(x)$ equals the Cotton invariant $C_{\widetilde{P}}(x)$ at $x$. Consequently, since the moment $I_{0}$ as well as the Cotton invariant are conformal invariants, we obtain $I_{0}(P)(x)=-\frac{1}{2} C_{P}(x)$. Finally, we conclude

$$
I_{0}=-\frac{1}{2} C
$$

\subsubsection{The moment of order 1}

We will proceed in the same manner as in the previous section. Without loss of generality we will start with an $x$-adapted metric and prove that the result is conformally invariant. We will use the same notation as above except we will omit the index $P$ and write $\nabla, H, \ldots$ instead of $\nabla^{P}, H_{P}, \ldots$ Again, by Propositions 6.13 and 6.14

$$
I_{1}(P)(x)=\mathcal{T}\left(d U_{1}(x, x)-\frac{1}{2} d I_{0}(P)(x)\right)
$$

and

$$
d U_{1}(x, x)=-\frac{1}{4} d P \mathcal{P}(x, x)=-\frac{1}{4} \sum_{I=1}^{m} u_{I}^{*}(x) \otimes\left(d \Delta u_{I}(x)+d H(x) u_{I}(x)\right)
$$


Let $u \in \Gamma(E)$ be a section in $E$ parallel along geodesics through $x$. In particular, $d^{k} u(x)=0, k>0$. Then for $Y \in T M$

$$
\begin{aligned}
d \Delta u(x)(Y) & =-\nabla_{Y} \sum_{i=1}^{4} \varepsilon_{i}\left(\nabla_{s_{i}} \nabla_{s_{i}} u+\nabla_{\nabla_{s_{i}} s_{i}} u\right)(x) \\
& =-\sum_{i=1}^{4} \varepsilon_{i}\left(\nabla_{Y} \nabla_{s_{i}} \nabla_{s_{i}} u+\nabla_{Y} \nabla_{\nabla_{s_{i}} s_{i}} u\right)(x)
\end{aligned}
$$

Now we extend $Y$ by parallel displacement along geodesics through $x$ and obtain from $d^{3} u(x)=0$ the equation

$$
\left(\nabla_{Y} \nabla_{s_{i}} \nabla_{s_{i}} u+\nabla_{s_{i}} \nabla_{Y} \nabla_{s_{i}} u+\nabla_{s_{i}} \nabla_{s_{i}} \nabla_{Y} u\right)(x)=0 .
$$

We have

$$
\begin{aligned}
\left(\nabla_{s_{i}} \nabla_{Y} \nabla_{s_{i}} u\right)(x) & =\left(\nabla_{Y} \nabla_{s_{i}} \nabla_{s_{i}} u+F\left(s_{i}, Y\right) \nabla_{s_{i}} u+\nabla_{\left[s_{i}, Y\right]} \nabla_{s_{i}} u\right)(x) \\
& =\left(\nabla_{Y} \nabla_{s_{i}} \nabla_{s_{i}} u\right)(x)
\end{aligned}
$$

where $F$ denotes the curvature of $\nabla$ and

$$
\begin{aligned}
& \nabla_{s_{i}} \nabla_{s_{i}} \nabla_{Y} u \stackrel{x}{=} \nabla_{s_{i}}\left(\nabla_{Y} \nabla_{s_{i}} u+F\left(s_{i}, Y\right) u+\nabla_{\left[s_{i}, Y\right]} u\right) \\
& \quad \stackrel{x}{=} \nabla_{s_{i}} \nabla_{Y} \nabla_{s_{i}} u+\left(\nabla_{s_{i}} F\right)\left(s_{i}, Y\right) u+F\left(\nabla_{s_{i}} s_{i}, Y\right) u+F\left(s_{i}, \nabla_{s_{i}} Y\right) u+\nabla_{s_{i}} \nabla_{\left[s_{i}, Y\right]} u \\
& \quad \stackrel{x}{=} \nabla_{Y} \nabla_{s_{i}} \nabla_{s_{i}} u+\left(\nabla_{s_{i}} F\right)\left(s_{i}, Y\right) u+\nabla_{s_{i}} \nabla_{\left[s_{i}, Y\right]} u .
\end{aligned}
$$

Since

$$
\nabla_{s_{i}} \nabla_{\left[s_{i}, Y\right]} u(x)=\left(\nabla_{\left.\left[s_{i},\left[s_{i}, Y\right]\right]^{u}\right)(x)+F\left(s_{i},\left[s_{i}, Y\right]\right) u(x)=0}\right.
$$

equation (43) now implies

$$
3 \nabla_{Y} \nabla_{s_{i}} \nabla_{s_{i}} u(x)+\left(\nabla_{s_{i}} F\right)\left(s_{i}, Y\right) u(x)=0 .
$$

On the other hand we get

$$
\nabla_{Y} \nabla_{\nabla_{s_{i}} s_{i}} u(x)=\nabla_{\left[Y, \nabla_{s_{i}} s_{i}\right]} u(x)+F\left(Y, \nabla_{s_{i}} s_{i}\right) u(x)=0 .
$$

Combining (42), (45) and (46) it turns out that

$$
d \Delta u(x)(Y)=\frac{1}{3} \sum \varepsilon_{i}\left(\nabla_{s_{i}} F\right)\left(s_{i}, Y\right) u(x)=-\frac{1}{3} \delta F(Y) u(x) .
$$

Consequently,

$$
d U_{1}(x, x)=\frac{1}{12} \delta F(x)-\frac{1}{4} d H(x) .
$$

Furthermore, because of $d R(x)=0$ we have

$$
d I_{0}(P)(x)=-\frac{1}{2} d C(x)=-\frac{1}{2} d H(x)
$$

and conclude

$$
I_{1}(P)(x)=\mathcal{T}\left(\frac{1}{12} \delta F\right)(x) .
$$

Since $\delta F$ is obviously trace free and conformally invariant we obtain

$$
I_{1}=\frac{1}{12} \delta F .
$$




\subsubsection{The moment of order 2}

The formula for $I_{2}$ will include a conformal invariant tensor on $M^{4}$ not discussed until now. Therefore, the first item of this section will be the introduction of this tensor and the description of some of its properties. However, let us first recall some facts about the Weyl curvature. The Weyl curvature of $\left(M^{4}, g\right)$ is defined to be the tensor $W=\mathcal{R}-g \bigotimes L \in \Gamma\left(\left(T^{*} M\right)^{4}\right)$, where $\oslash$ denotes the Kulkarni - Nomizu product (see [Bes87]). In the following all traces refer to the metric $g$ which is under consideration.

Proposition 6.15 1. $W(X, Y, U, V)=W(U, V, X, Y)=-W(Y, X, U, V)$ for all $X, Y, U, V \in T M$.

2. $\operatorname{Trace}_{(1,4)} W=0$

3. $\operatorname{Trace} Q_{4}(W, W)=\frac{1}{8} g \cdot\|W\|^{2}$

4. $\sum \varepsilon_{j}\left(\nabla_{s_{j}} W\right)\left(X, Y, Z, s_{j}\right)=-\left(\nabla_{X} L\right)(Y, Z)+\left(\nabla_{Y} L\right)(X, Z)$ for all $X, Y, Z \in T M$ and a pseudo-orthonormal frame $s_{1}, \ldots, s_{4}$ with $\varepsilon_{i}=g\left(s_{i}, s_{i}\right)$.

Definition 6.18 The symmetric tensor $\mathcal{B} \in \Gamma\left(S^{2} T^{*} M\right)$ defined by

$$
\mathcal{B}:=S \operatorname{Trace}_{(1,3)(2,6)} \mathfrak{C}^{2} W
$$

is called the Bach tensor of $\left(M^{4}, g\right)$.

Similarly to $Q_{4}$ we define for $A \in \Gamma\left(\left(T^{*} M\right)^{2}\right)$ and $B \in \Gamma\left(\left(T^{*} M\right)^{4}\right)$

$$
Q_{2,4}(A, B):=S \operatorname{Trace}_{(1,3)(2,6)}(A \otimes B) .
$$

Then we have

Proposition 6.16 1. $\mathcal{B}=S \operatorname{Trace}_{(1,3)(2,6)} \nabla^{2} W-Q_{2,4}(L, W)$

2. $\mathcal{B}$ is trace free.

3. $\mathcal{B}$ is conformally invariant of weight -1 .

Proof. Since $W$ is conformally invariant $\mathfrak{X} W=0$ holds. Thus, $\mathfrak{C} W=\nabla W$. From Theorem 6.1 we now obtain

$$
\mathfrak{X C} W=\mathfrak{C} \mathfrak{X} W+K W+2 E W=K W+2 E W .
$$

Finally,

$$
\begin{aligned}
\mathfrak{C}^{2} W & =\nabla \mathfrak{C} W-L\lrcorner \mathfrak{X C} W \\
& \left.\left.=\nabla^{2} W-L\right\lrcorner K W-2 L\right\lrcorner E W \\
& \left.=\nabla^{2} W-L\right\lrcorner K W-2 L \otimes W
\end{aligned}
$$


It is a straightforward calculation using the definition of the map $K$ and the symmetry properties of the Weyl curvature to verify the equality

$$
\left.\operatorname{Trace}_{(1,3)(2,6)}(L\lrcorner K W\right)=Q_{2,4}(L, W) .
$$

This yields assertion 1 . Because of the symmetry properties of $W$ we have $Q_{2,4}(L, W)=$ $\operatorname{Trace}_{(1,3)(2,6)}(L \otimes W)$. We obtain

$$
\begin{aligned}
\operatorname{Trace}_{2,4}(L, W) & =\operatorname{Trace} \operatorname{Trace}_{(1,3)(2,6)}(L \otimes W)=\operatorname{Trace}_{(1,3)(2,6)(4,5)}(L \otimes W) \\
& =0
\end{aligned}
$$

where the last equality follows from $\operatorname{Trace}_{(1,4)} W=0$. Similarly, this property of $W$ yields

$$
\text { Trace } \operatorname{Trace}_{(1,3)(2,6)} \nabla^{2} W=0
$$

and assertion 2. follows.

Note that each mapping $T$ with simple transformation satisfies $\mathfrak{X}$ Trace $T=$ Trace $\mathfrak{X} T$.

As we observed in Section 6.2 the conformal gauge derivative $\mathfrak{C} W$ has again a simple transformation law with weight $\omega=1$. By Prop. 6.1 it suffices to prove

$$
\mathfrak{X T r a c e}_{(1,3)(2,6)} \mathfrak{C C W}=\operatorname{Trace}_{(1,3)(2,6)} \mathfrak{X} \mathfrak{C C} W=0 .
$$

From Theorem 6.1 we see

$$
\begin{aligned}
\mathfrak{X} \mathfrak{C} W & =\mathfrak{C X C} W+K \mathfrak{C} W+2 E \mathfrak{C} W \\
& =\mathfrak{C}(K W+2 E W)+K \mathfrak{C} W+2 E \mathfrak{C} W
\end{aligned}
$$

Consequently,

$$
\begin{aligned}
\mathfrak{X C C} & \left(\sigma, Z_{1}, Z_{2}, X, Y, U, V\right)= \\
= & \mathfrak{C}_{Z_{1}} K W\left(\sigma, Z_{2}, X, Y, U, V\right)+2 \mathfrak{C}_{Z_{1}} E W\left(\sigma, Z_{2}, X, Y, U, V\right) \\
& +\left(K\left(\sigma, Z_{1}\right) \cdot \mathfrak{C} W\right)\left(Z_{2}, X, Y, U, V\right)+2 \sigma\left(Z_{1}\right) W(X, Y, U, V) \\
= & \left(K\left(\sigma, Z_{2}\right) \cdot \nabla_{Z_{1}} W\right)(X, Y, U, V)+2 \sigma\left(Z_{2}\right)\left(\nabla_{Z_{1}} W\right)(X, Y, U, V) \\
& +\left(\nabla_{-K\left(\sigma, Z_{1}\right) Z_{2}} W\right)(X, Y, U, V)+\left(K\left(\sigma, Z_{1}\right) \cdot \nabla_{Z_{2}} W\right)(X, Y, U, V) \\
& +2 \sigma\left(Z_{1}\right)\left(\nabla_{Z_{2}} W\right)(X, Y, U, V)
\end{aligned}
$$

where we used Propositions 6.5 and 6.6 and $\mathfrak{C} W=\nabla W$. A simple calculation using the definition of $K$ and the symmetry properties of $W$ now implies

$$
\operatorname{Trace}_{(1,3)(2,6)} \mathfrak{X C C W}=0
$$

which proves assertion 3 . 
Proposition 6.17 Let $g$ be an $x$-adapted metric on $M^{4}, x \in M^{4}$. Then the Bach tensor $\mathcal{B}$ associated to this metric satisfies

$$
\mathcal{B} \stackrel{x}{=}-\frac{5}{3} d^{2} R \quad \bmod g
$$

Proof. Let $s_{1}, \ldots, s_{4}$ be as usual a local pseudo-orthonormal frame with $\varepsilon_{i}=g\left(s_{i}, s_{i}\right)$ and $Y, Z$ vector fields on $M^{4}$ and let all fields be parallel along geodesics through $x$. From Proposition 6.15 assertion 4 we obtain

$$
\begin{aligned}
\sum_{i, j} \varepsilon_{i} \varepsilon_{j}\left(\nabla_{s_{i}} \nabla_{s_{j}} W\right)\left(s_{i}, Y, Z, s_{j}\right) & \stackrel{x}{=} \sum_{i} \varepsilon_{i} s_{i}\left(\sum_{j} \varepsilon_{j}\left(\nabla_{s_{j}} W\right)\left(s_{i}, Y, Z, s_{j}\right)\right) \\
& \stackrel{x}{=} \sum_{i} \varepsilon_{i} s_{i}\left(-\left(\nabla_{s_{i}} L\right)(Y, Z)+\left(\nabla_{Y} L\right)\left(s_{i}, Z\right)\right) \\
& \stackrel{x}{=} \sum_{i} \varepsilon_{i}\left(-\left(\nabla_{s_{i}} \nabla_{s_{i}} L\right)(Y, Z)+\left(\nabla_{s_{i}} \nabla_{Y} L\right)\left(s_{i}, Z\right)\right) \\
& \stackrel{x}{=} \sum_{i} \varepsilon_{i}\left(-\left(\nabla_{s_{i}} \nabla_{s_{i}} L\right)(Y, Z)+\left(\nabla_{Y} \nabla_{s_{i}} L\right)\left(s_{i}, Z\right)\right)
\end{aligned}
$$

where the last equality uses $L(x)=0$. Hence,

$$
S \operatorname{Trace}_{(1,3)(2,6)} \nabla^{2} W \stackrel{x}{=} \Delta L-d \delta L .
$$

the first term of the r.h.s. equals

$$
\Delta L=\frac{1}{12}(\nabla R) \cdot g-\frac{1}{2} \Delta(\mathrm{Ric})
$$

which follows from $L=\frac{1}{12} R g-\frac{1}{2}$ Ric and $\nabla g=0$. Now turn to the second term. By

$$
\delta(R \cdot g)(X)=-\sum_{i} \varepsilon_{i} \nabla_{s_{i}}(R \cdot g)\left(s_{i}, X\right)=-\sum_{i} \varepsilon_{i} d R\left(s_{i}\right) \cdot g\left(s_{i}, X\right)=-d R(X)
$$

we get

$$
d \delta R g=-d^{2} R
$$

Furthermore, $\delta \mathrm{Ric}=-\frac{1}{2} d R$ yields

$$
d \delta \text { Ric }=-\frac{1}{2} d^{2} R
$$

Consequently,

$$
d \delta L=-\frac{1}{12} d^{2} R+\frac{1}{4} d^{2} R=\frac{1}{6} d^{2} R .
$$

On the other hand we have $Q_{2,4}(W, L) \stackrel{x}{=} 0$ since $L(x)=0$. Hence, by definition

$$
\mathcal{B} \stackrel{x}{=} S \operatorname{Trace}_{(1,3)(2,6)} \nabla^{2} W .
$$


Combining this with (47), (48) and (49) we conclude

$$
\mathcal{B} \stackrel{x}{=} \frac{1}{12}(\Delta R) \cdot g-\frac{1}{2} \Delta(\mathrm{Ric})-\frac{1}{6} d^{2} R \stackrel{x}{=}-\frac{1}{2} \Delta(\mathrm{Ric})-\frac{1}{6} d^{2} R .
$$

Now the term $\Delta$ (Ric) remains to be discussed. As we observed the Ricci tensor of an $x$-adapted metric satisfies $d^{2} \operatorname{Ric} \stackrel{x}{=} \frac{2}{9} Q_{4}(\mathcal{R}, \mathcal{R})$. Since $\mathcal{R}=W+g \otimes L$ and $L(x)=0$ we get

$$
d^{2} \operatorname{Ric} \stackrel{x}{=} \frac{2}{9} Q_{4}(W, W) .
$$

Consequently, by Lemma 6.15 , assertion 3

$$
\operatorname{Trace} d^{2} \operatorname{Ric} \stackrel{x}{=} \frac{2}{9} \operatorname{Trace} Q_{4}(W, W)=\frac{1}{8}\|W\|^{2} g \stackrel{x}{=} 0 .
$$

On the other hand,

$$
\begin{aligned}
& \operatorname{Trace} d^{2} \operatorname{Ric}(X, Y)=\sum_{i} \varepsilon_{i}\left(d^{2} \operatorname{Ric}\right)\left(s_{i}, s_{i}, X, Y\right)= \\
& =\frac{1}{4 !} \sum_{i} \varepsilon_{i}\left(4\left(\nabla_{s_{i}} \nabla_{s_{i}} \operatorname{Ric}\right)(X, Y)+4\left(\nabla_{s_{i}} \nabla_{X} \operatorname{Ric}\right)\left(s_{i}, Y\right)+4\left(\nabla_{X} \nabla_{s_{i}} \operatorname{Ric}\right)\left(s_{i}, Y\right)\right. \\
& +4\left(\nabla_{s_{i}} \nabla_{Y} \operatorname{Ric}\right)\left(s_{i}, X\right)+4\left(\nabla_{Y} \nabla_{s_{i}} \operatorname{Ric}\right)\left(s_{i}, X\right) \\
& \left.+2\left(\nabla_{X} \nabla_{Y} \operatorname{Ric}\right)\left(s_{i}, s_{i}\right)+2\left(\nabla_{Y} \nabla_{X} \operatorname{Ric}\right)\left(s_{i}, s_{i}\right)\right)
\end{aligned}
$$

Since Ric $(x)=0$ implies $\nabla_{s_{i}} \nabla_{X} \operatorname{Ric} \stackrel{x}{=} \nabla_{X} \nabla_{s_{i}}$ Ric this equals

$$
\begin{aligned}
& =-\frac{1}{6}(\Delta \operatorname{Ric})(X, Y)-\frac{2}{3} d \delta \operatorname{Ric}(X, Y)+\frac{1}{6} d^{2} R(X, Y) \\
& =-\frac{1}{6}(\Delta \operatorname{Ric})(X, Y)+\frac{1}{2} d^{2} R(X, Y)
\end{aligned}
$$

using again $\delta$ Ric $=-\frac{1}{2} d R$. Equations (51) and (52) now yield

$$
-\Delta \mathrm{Ric}+3 d^{2} R \stackrel{x}{\equiv} 0 \bmod g
$$

and Equation (50) implies

$$
\mathcal{B} \stackrel{x}{=}-\frac{3}{2} d^{2} R-\frac{1}{6} d^{2} R=-\frac{5}{3} d^{2} R \bmod g .
$$

Now we are able to calculate $I_{2}(P)$ for a normally hyperbolic operator $P$ on $M^{4}$. Again let without loss of generality the associated Lorentz metric $g$ be $x$-adapted for a fixed $x \in M^{4}$. Propositions 6.13 and 6.14 yield

$$
I_{2}(P)(x)=\mathcal{T}\left(-\frac{1}{6} d^{2} P \mathcal{P}(x, x)-d I_{1}(P)(x)-\frac{1}{3} d^{2} I_{0}(P)(x)\right)
$$


Since $I_{1}=\frac{1}{12} \delta F$ and $I_{0}=-\frac{1}{2} C$ as we have seen in the previous sections we get

$$
I_{2}(P)(x)=\mathcal{T}\left(-\frac{1}{6} d^{2} P \mathcal{P}(x, x)-\frac{1}{12} d \delta F+\frac{1}{6} d^{2} C\right)
$$

With the same notation as above we have

$$
d^{2} P \mathcal{P}(x, x)=\sum_{I} u_{I}^{*}(x) \otimes d^{2} P u_{I}(x)=\sum_{I} u_{I}^{*}(x) \otimes d^{2} \Delta u_{I}(x)+d^{2} H(x) .
$$

By definition of the Cotton invariant

$$
d^{2} H=d^{2}\left(C+\frac{1}{6} R\right)=d^{2} C+\frac{1}{6} d^{2} R .
$$

Proposition 6.17 now implies

$$
d^{2} H \stackrel{x}{=} d^{2} C-\frac{1}{10} \mathcal{B} \bmod g
$$

Consequently,

$$
I_{2}(P)(x)=\mathcal{T}\left(-\frac{1}{6} \sum_{I} u_{I}^{*}(x) \otimes d^{2} \Delta u_{I}(x)+\frac{1}{60} \mathcal{B}-\frac{1}{12} d \delta F\right)
$$

Consider now the first term of the r.h.s. Here we proceed in the same manner as in Section 6.6.4. Let $Y$ be a vector in $x$ extended by parallel displacement along geodesics to a local vector field. Furthermore, let $u$ be a section parallel along geodesics through $x$. In particular, we have $d^{4} u(x)=0$. Consequently,

$$
\begin{aligned}
& \left(\nabla_{s_{i}} \nabla_{s_{i}} \nabla_{Y} \nabla_{Y}+\nabla_{s_{i}} \nabla_{Y} \nabla_{s_{i}} \nabla_{Y}+\nabla_{Y} \nabla_{s_{i}} \nabla_{s_{i}} \nabla_{Y}\right. \\
& \left.\quad+\nabla_{Y} \nabla_{s_{i}} \nabla_{Y} \nabla_{s_{i}}+\nabla_{Y} \nabla_{Y} \nabla_{s_{i}} \nabla_{s_{i}}+\nabla_{s_{i}} \nabla_{Y} \nabla_{Y} \nabla_{s_{i}}\right) u \stackrel{x}{=} 0
\end{aligned}
$$

Commuting the derivatives we get

$$
\begin{aligned}
0 \stackrel{x}{=} & 6 \nabla_{Y} \nabla_{Y} \nabla_{s_{i}} \nabla_{s_{i}} u+3\left(\nabla_{Y} \nabla_{s_{i}} F\right)\left(s_{i}, Y\right) u+8 \nabla_{Y} \nabla_{s_{i}} \nabla_{s_{i}} u \\
& -4 \nabla_{\nabla_{s_{i}}} \nabla_{Y s_{i}} \nabla_{Y} u+6 F\left(s_{i}, Y\right) \nabla_{Y} \nabla_{s_{i}} u+6 F\left(s_{i}, \nabla_{Y} \nabla_{s_{i}} Y\right) u
\end{aligned}
$$

Extending $\nabla_{Y} \nabla_{s_{i}} Y(x)$ parallel along geodesics starting at $x$ and using $d^{2} u(x)=0$ we obtain

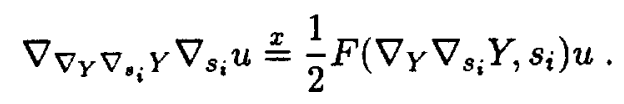

Furthermore, $\nabla_{Y} \nabla_{s_{i}} Y=\frac{1}{2} \mathcal{R}\left(Y, s_{i}\right) Y$ and $\nabla_{Y} \nabla_{s_{i}} u=\frac{1}{2} F\left(Y, s_{i}\right) u$. After summation (54) now yields

$$
\begin{aligned}
0 \stackrel{x}{=} & 6 \sum_{i} \varepsilon_{i} \nabla_{Y} \nabla_{Y} \nabla_{s_{i}} \nabla_{s_{i}} u-3 d \delta F(Y, Y)+4 \nabla_{R i c(Y)} \nabla_{Y} u \\
& -3 \sum_{i} \varepsilon_{i} F\left(s_{i}, Y\right) F\left(s_{i}, Y\right) u-\sum_{i} \varepsilon F\left(\mathcal{R}\left(Y, s_{i}\right) Y, s_{i}\right) u .
\end{aligned}
$$


The last term of (55) vanish because of the symmetry properties of $\mathcal{R}$ and $F$. Finally, using $\operatorname{Ric}(x)=0$ we conclude

$$
\sum_{i} \varepsilon_{i} \nabla_{Y} \nabla_{Y} \nabla_{s_{i}} \nabla_{s_{i}} u \stackrel{x}{=} \frac{1}{2} d \delta F(Y, Y)-\frac{1}{2} \sum_{i} \varepsilon_{i} F\left(s_{i}, Y\right) F\left(s_{i}, Y\right) u .
$$

In the same way by commuting the derivatives and using again $\operatorname{Ric}(x)=0$ we get

$$
\nabla_{Y} \nabla_{Y} \nabla_{\nabla_{s_{i}} s_{i}} u \stackrel{x}{=} 0 \text {. }
$$

Defining

$$
Q_{2}(F, F):=S \operatorname{Trace}_{(1,3)} F \otimes F
$$

we obtain

$$
d^{2} \Delta u(x)=-\frac{1}{2} d \delta F u(x)-\frac{1}{2} Q_{2}(F, F) u(x) .
$$

Now we are ready to finish the calculation of $I_{2}$. Combining (53) with (56) yields

$$
I_{2} \stackrel{x}{=} \frac{1}{12} \mathcal{T}\left(Q_{2}(F, F)+\frac{1}{5} \mathcal{B}\right) \text {. }
$$

We already know that the Bach tensor is trace free. On the other hand we have

$$
\begin{aligned}
\mathcal{T}\left(Q_{2}(F, F)\right) & =Q_{2}(F, F)-\frac{1}{4} g \otimes \operatorname{Trace} Q_{2}(F, F) \\
& =Q_{2}(F, F)-\frac{1}{4} g \otimes\|F\|^{2}
\end{aligned}
$$

It turns out that

$$
I_{2}=\frac{1}{12}\left(Q_{2}(F, F)-\frac{1}{4} g \otimes\|F\|^{2}+\frac{1}{5} \mathcal{B} \otimes \operatorname{Id}_{E}\right) .
$$

Collecting the above proven results one obtains the following geometric conditions for a normally hyperbolic operator to be of Huygens type:

Theorem 6.11 Let $P: \Gamma(E) \longrightarrow \Gamma(E)$ be a normally hyperbolic operator of Huygens type on a 4-dimensional manifold $M^{4}$. Let $g$ be the Lorentzian metric defined by $P$, $\nabla^{P}$ the covariant derivative and $H_{P}$ the homomorphism on $E$ associated to $P$ by its Weitzenböck formula. Then the following conditions are satisfied:

1. $H_{P}=\frac{1}{6} R$, where $R$ is the scalar curvature of $\left(M^{4}, g\right)$.

2. $\nabla^{P}$ is a Yang-Mills connection: for the curvature $F^{P}$ of $\nabla^{P} \quad \delta F^{P}=0$ holds.

3. The Bach tensor $\mathcal{B}$ of $\left(M^{4}, g\right)$ equals a multiple of the "energy impuls tensor" of $F^{P}$ :

$$
\mathcal{B} \otimes I d_{E}=-5\left(Q_{2}\left(F^{P}, F^{P}\right)-\frac{1}{4} g \otimes\left\|F^{P}\right\|^{2}\right)
$$

Finally we remark that formulas for the moments $I_{3}(P)$ and $I_{4}(P)$ for normally hyperbolic operators on 4-dimensional manifolds can be found in the appendix of [Gün88]. 


\section{Applications}

In this section we will describe some results concerning the Huygens property for the Dirac, the Hodge-Laplace and the Yamabe operator.

In the study of Huygens operators a certain class of Lorentzian manifolds plays an extra-ordinary role, the so-called plane wave manifolds (see [Sch74] for detailed explanations).

Definition 7.1 A Lorentzian manifold $\left(M^{n}, g\right)$ is called plane wave manifold if the following conditions are satisfied:

1. There exists an isotropic parallel vector field $V \in \mathfrak{X}(M)$.

2. The curvature tensor $\mathcal{R}$ of $(M, g)$ satisfies

$$
\operatorname{Trace}_{(3,5),(4,6)} \mathcal{R} \otimes \mathcal{R}=0
$$

3. $\mathcal{R}$ is quasi recurrent with $V$ i.e. that there exists a $(4,0)$-tensor field $\mathcal{R}_{1}$ such that

$$
\nabla \mathcal{R}=V^{\mathrm{b}} \otimes \mathcal{R}_{1}
$$

where $V^{\mathrm{b}}$ denotes the 1 -form dual to $V$ with respect to $g$.

A plane wave manifold is foliated by submanifolds of codimension 1 , the integral curves of $V$ are isotropic geodesics running in the leaves of the foliation and $M$ is locally symmetric along the leaves: $\nabla_{X} \mathcal{R}=0$ for all vectors $X$ tangent to the leaves of the foliation.

The scalar curvature of a plane wave manifold is zero. A 4-dimensional plane wave manifold is of Petrov type $N$ in points where the Weyl tensor differs from zero.

Proposition 7.1 A Lorentzian manifold $\left(M^{n}, g\right)$ is a plane wave manifold with the parallel isotropic vector field $V$ if and only if for each point $x \in M$ there exists a coordinate neighbourhood $\left(U,\left(x^{1}, \ldots, x^{n}\right)\right)$ such that the metric $g$ has the form

$$
g_{\mid U}=2 d x^{1} d x^{2}+a_{\alpha \beta}\left(x^{1}\right) d x^{\alpha} d x^{\beta} \quad 3 \leq \alpha, \beta \leq n
$$

where $\left(a_{\alpha \beta}\right)$ is a positive definite matrix, depending only on $x^{1}$ and $V$ is given by $\left.V\right|_{U}=\frac{\partial}{\partial x_{2}}$.

\subsection{Dirac operators}

Let $\left(M^{n}, g\right)$ be an oriented Lorentzian spin manifold with spinor bundle $S$ and denote by $D: \Gamma(S) \longrightarrow \Gamma(S)$ the Dirac operator of $\left(M^{n}, g\right)$ :

$$
D \varphi:=\sum_{j=1}^{n} \varepsilon_{j} s_{j} \cdot \nabla_{s_{j}}^{S} \varphi, \quad \varphi \in \Gamma(S),
$$

where $\left(s_{1}, \ldots, s_{n}\right)$ denotes a local orthonormal basis with timelike vector $s_{1}, \varepsilon_{j}=g\left(s_{j}, s_{j}\right)$ and $\cdot$ is the Clifford multiplication.

If $\left(M^{n}, g\right)$ is a plane wave manifold of even dimension $n$, then all Hadamard coefficients $U_{k}, k \geq 1$, of the square $D^{2}$ of the Dirac operator vanish (for a suitable covering by geodesically normal domains). Hence from the Hadamard criterion follows 
Theorem 7.1 ([Bau96])

Let $\left(M^{n}, g\right)$ be a plane wave manifold of even dimension $n \geq 4$ with a spinor bundle $S$. Then the square of the Dirac operator $D^{2}: \Gamma(S) \longrightarrow \Gamma(S)$ is Huygens.

Furthermore, comparing the square of the Dirac operator on Lorentzian space forms with the Yamabe operator one can prove

Theorem 7.2 (Bau96])

Let $D$ be the Dirac operator on a Lorentzian spin manifold of constant sectional curvature $K$. Then the operator $D^{2}-K$ is of Huygens type.

Dirac operators and other spinor field operators on 4-dimensional manifold were studied by V.Wünsch using the 2-component spinor calculus introduced by Infeld, van der Warden and Penrose (see [Wün78], [Wün79], [Wün80], [Wün85]). The curvature conditions arising from the vanishing of the moments allow a complete characterization of the 4-dimensional analytic Lorentzian spin manifolds on which the square of the Dirac operator is of Huygens type.

Theorem 7.3 ([Wün78] Folg.3.3, [Wün79] Prop.5.6, [Wün80] Prop. 2.11)

Let $D: \Gamma(S) \longrightarrow \Gamma(S)$ denote the Dirac operator of a 4-dimensional space and time oriented analytic Lorentzian spin manifold $\left(M^{4}, g\right)$ and let $f$ be a smooth function on $M$. If the operator $D^{2}-f: \Gamma(S) \longrightarrow \Gamma(S)$ is Huygens, then the scalar curvature $R$ of $(M, g)$ is constant and equals $12 f$.

If $R$ is constant and non zero, then $D^{2}-\frac{1}{12} R$ is Huygens if and only if $\left(M^{4}, g\right)$ has constant sectional curvature.

If $R$ is identically zero, then $D^{2}$ is Huygens if and only if $\left(M^{4}, g\right)$ is conformally flat or a plane wave manifold.

Now, let $G$ be a Lie group, let $P$ be a $G$-principal fibre bundle over $(M, g)$ and denote by $E:=P \times{ }_{G} V$ a complex vector bundle associated to $P$. Then each connection $A$ of $P$ defines a covariant derivative $\nabla^{A}$ in $E$. The Dirac operator $D_{A}$ coupled to the connection $A$ is defined by

$$
D_{A}(\varphi \otimes e):=D \varphi \otimes e+\sum_{j=1}^{n} \varepsilon_{j} s_{j} \cdot \varphi \otimes \nabla_{s_{j}}^{A} e .
$$

For Huygens operators on 4-dimensional manifolds the Cotton invariant vanishes. Therefore, one can reduce the study of the Huygens property for coupled Dirac operators on 4- dimensional manifolds to the uncoupled case.

Theorem 7.4 ([Ill88], [Bau96])

Let $D_{A}: \Gamma(S \otimes E) \longrightarrow \Gamma(S \otimes E)$ denote the Dirac operator over a 4-dimensional space and time oriented analytic Lorentzian spin manifold $\left(M^{4}, g\right)$ coupled to a connection $A$ and let $f$ be a smooth function on $M$.

If the operator $D_{A}^{2}-f$ is Huygens, then the scalar curvature $R$ of $\left(M^{4}, g\right)$ is constant 
and equals $12 f$. If $R$ is constant and non zero, then $D_{A}^{2}-\frac{1}{12} R$ is Huygens if and only if $\left(M^{4}, g\right)$ has constant sectional curvature and $A$ is flat.

If $R$ is identiaclly zero, then $D_{A}^{2}$ is Huygens if and only if $\left(M^{4}, g\right)$ is conformally flat or locally isometric to a plane wave manifold and $A$ is flat.

\subsection{Hodge-Laplace operators}

Let us denote by $\Delta_{p}: \Omega^{p}(M) \longrightarrow \Omega^{p}(M)$ the Hodge-Laplace operator on $p$-forms over an oriented Lorentzian manifold $\left(M^{n}, g\right)$.

For a plane wave manifold of dimension $n$ one can prove that all Hadamard coefficients $U_{k}^{(p)}, k \geq 2$, of the Hodge-Laplace operator $\Delta_{p}, p=1, \ldots, n-1$, vanish. Hence the Hadamard criterion yields

Theorem 7.5 ([Sch71], [Gün88], Lemma 8.3.6, Th.8.3.7)

Let $\left(M^{n}, g\right)$ be a plane wave manifold of even dimension $n \geq 6$. Then the Hodge-Laplace operators $\Delta_{p}$ on $p$-forms of $\left(M^{n}, g\right)$ are of Huygens type for all $p=1, \ldots, n-1$.

In the 4-dimensional analytic case there is a complete description of the Lorentzian manifolds with Huygens Hodge-Laplace operators:

Theorem 7.6 ([Sch78], [Wün79], [Gün88] Example to Th. 7.3.8, Rem. 7.3.9, Prop. 7.4.10)

Let $\left(M^{4}, g\right)$ be a 4-dimensional oriented Lorentzian manifold. Then

1. The Hodge-Laplace operators $\Delta_{1}$ and $\Delta_{3}$ are Huygens if and only if $\left(M^{4}, g\right)$ is flat.

2. Let $\left(M^{4}, g\right)$ be analytic. Then $\Delta_{2}$ is Huygens if and only if $\left(M^{4}, g\right)$ is conformally flat with vanishing scalar curvature.

\subsection{The Yamabe operator}

Let $Y_{g}:=\Delta_{0}+\frac{n-2}{4(n-1)} R: C^{\infty}(M) \longrightarrow C^{\infty}(M)$ be the Yamabe operator of a Lorentzian manifold $(M, g)$. The Yamabe operator is conformally invariant:

$$
Y_{\tilde{g}}=e^{\frac{-(n+2)}{2} \varphi} Y_{g} e^{\frac{n-2}{2} \varphi} \quad, \quad \tilde{g}=e^{2 \varphi} g
$$

On conformally flat Lorentzian manifolds of even dimension $n \geq 4$ the Yamabe operator is of Huygens type, since it is trivial. Moreover, for Lorentzian space forms the following result was proved

Theorem 7.7 ([Hel94] chap. 5.5.4, [SS94])

Let $\left(M^{n}, g\right)$ be a Lorentzian manifold of constant sectional curvature and even dimension $n \geq 4$. Then for each $m=3,5, \ldots n-1$ the shifted Laplace operator

$$
L_{m}:=\Delta_{0}+K(n-m)(m-1)
$$

is of Huygens type. 
For plane wave manifolds $\left(M^{n}, g\right)$ all Hadamard coefficients $U_{k}, k \geq 1$, of the Yamabe operator vanish. Hence, using the Hadamard criterion and the conformal invariance of the Yamabe operator one obtains

Theorem 7.8 ([Gün65], [Gün88] Th. 8.3.4)

Let $\left(M^{n}, g\right)$ be a Lorentzian manifold of even dimension $n \geq 4$. If $\left(M^{n}, g\right)$ is locally conformal equivalent to a plane wave manifold, then the Yamabe operator of $\left(M^{n}, g\right)$ is Huygens.

Contrary to the case of the Dirac and the Hodge-Laplace operators on $p$-forms, there is no complete description of the class of 4-dimensional manifolds on which the Yamabe operator is Huygens. All known results suggest the conjecture:

Conjecture: The Yamabe operator on a 4-dimensional Lorentzian manifold $\left(M^{4}, g\right)$ is of Huygens type if and only if $\left(M^{4}, g\right)$ is locally conformal equivalent to a plane wave manifold.

Several special classes of 4-dimensional manifolds were studied:

Theorem 7.9 ([McL69], [Wün79], [CM86], [CM87], [CM88], [Gün88] chap.8, [Wün89], [CCMW91], [AM93], [AM94], [Wün94])

Let $\left(M^{4}, g\right)$ be a 4-dimensional Lorentzian manifold such that the Yamabe operator $Y$ of $\left(M^{4}, g\right)$ is Huygens. Then:

1. If $\left(M^{4}, g\right)$ is Ricci-flat and not locally symmetric, then it is a plane wave manifold.

2. If $\left(M^{4}, g\right)$ is an Einstein space, then it is a plane wave manifold or a space of constant sectional curvature.

3. If $(M, g)$ is locally symmetric or if the Ricci tensor is parallel, then it is conformally flat or a plane wave manifold.

4. If $\left(M^{4}, g\right)$ is of Petrov type $N$, then it is locally conformal equivalent to a plane wave manifold.

5. $\left(M^{4}, g\right)$ is not of Petrov type D.

6. If $\left(M^{4}, g\right)$ is conform recurrent, then $\left(M^{4}, g\right)$ is a plane wave manifold.

7. If $\left(M^{4}, g\right)$ is recurrent then it is conformally flat or a plane wave manifold.

8. If $\left(M^{4}, g\right)$ is $(2 \times 2)$-decomposable or central symmetric, then it is conformally flat.

9. If the divergence of the Weyl tensor vanishes, then $\left(M^{4}, g\right)$ is locally conformal equivalent to a plane wave manifold.

\subsection{The wave operator of Riemannian symmetric spaces}

Let $\left(X^{m}, h\right)$ be a Riemannian symmetric space and denote by $\Delta_{X}$ the Laplace operator acting on functions over $X$. Let $\left(M=\mathbb{R} \times X, g=-d t^{2}+h\right)$ be the Lorentzian product of $X$ with the real line. We consider the wave operator $\square_{X}:=\frac{\partial^{2}}{\partial t^{2}}+\Delta_{X}: C^{\infty}(M) \longrightarrow$ $C^{\infty}(M)$. The Huygens property for this wave operator was studied by Helgason, Olafsson, Schlichtkrull et al deriving explicit formulas for the fundamental solution using methods of harmonic analysis. As already stated, the shifted wave operator 
$\square_{X}+\frac{(m-1)^{2}}{4} K$ for an $m$-dimensional space $X$ of constant sectional curvature $K$ is trivial, hence for odd $m \geq 3$ this operator is of Huygens type.

For Riemannian symmetric spaces of non-compact type the following is known:

Theorem 7.10 ([Hel92], [OS92], [Hel94], chap.5.5)

Let $X=G / K$ be an odd-dimensional Riemannian symmetric space of noncompact type and suppose that all Cartan subgroups of $G$ are conjugated. Denote by $\|\rho\|$ the length of the half sum of all positive roots with respect to the Killing form. Then the shifted wave operator $\square_{X}-\|\rho\|^{2}$ is of Huygens type.

For Riemannian symmetric spaces of compact type the following is known:

Theorem 7.11 (Feg84], [Hel84], [Hel94] chap. 5.5)

If $X$ is an odd-dimensional, compact semi-simple Lie group with the metric given by the negative Killing form, then the shifted wave operator $\square_{X}+\frac{\text { dimX }}{24}$ is of Huygens type.

\subsection{The D'Alambert operator plus potentials on the Minkowski space}

We know that the D'Alambert operator $\square_{m}=\frac{\partial^{2}}{\partial t^{2}}-\sum_{i=1}^{m} \frac{\partial^{2}}{\partial x_{i}^{2}}$ acting on functions over an open subset of the Minkowski space $\mathbb{R} \times \mathbb{R}^{m}$ is of Huygens type if and only if $m$ is odd and $m \geq 3$. In dimension $m=3$ we can not add a potential $V$, such that the operator $\square_{3}+V$ remains Huygens since the Cotton invariant has to vanish. The question which potentials can be added in higher dimension was studied by Stellmacher, Lagnese, Schimming, Berest, Veselov and others (see [BV94b] for a detailed survey).

Using so-called Dunkl operators it can be checked that for a certain class of potentials $V$ the Hadamard coefficient $U_{\frac{m-1}{2}}$ of $\square_{m}+V$ vanishes.

Theorem 7.12 ([BV94a]) Let $G$ be a Coxeter group in $\mathbb{R}^{p}, 0<p \leq m$, and denote by $\mathcal{R}^{+}$the set of all positive roots of $G$. For a $G$-invariant function $\mu: \mathcal{R}^{+} \longrightarrow \mathbb{N}$ we denote by $V_{\mu}$ the function

$$
V\left(x_{1}, \ldots, x_{p}\right):=\sum_{\alpha \in \mathcal{R}^{+}} \frac{\mu(\alpha)(\mu(\alpha)+1)\langle\alpha, \alpha\rangle}{\langle\alpha, x\rangle^{2}}
$$

Then the operator $P:=\square_{m}+V_{\mu}\left(x_{1}, \ldots, x_{p}\right)$, acting on functions over the Minkowski space without the hypersurfaces in $\mathbb{R}^{p}$ orthogonal to the roots is of Huygens type if $m>3+2 \sum_{\alpha \in R^{+}} \mu(\alpha)$.

\section{References}

[AM93] W.G. Anderson and R.G. McLenaghan. On Hygens' principle for relativistic wave equations. C.R.Math. Acad. Sci Soc. R. Can., 15(1):41-45, 1993. 
[AM94] W.G. Anderson and R.G. McLenaghan. On the validity of Hygens' principle for second order partial differential equations with four independent variables. II: A sixth necessary condition. Ann. Inst. Henri Poincare, Phys. Theor., 60(4):373-432, 1994.

[Bau96] H. Baum. The Dirac operator on Lorentzian spin manifolds and the Huygens property. SFB 288-Preprint, 1996.

[Bes87] A.L. Besse. Einstein Manifolds, volume 10 of Ergebnisse der Mathematik und ihrer Grenzgebiete. Springer, 1987.

[BGM74] M. Berger, P. Gauduchon, and E. Mazet. Le Spectre d'une Variete Riemanniennes, volume 194 of Lecture Notes in Math. Springer, 1974.

[BV94a] Y.Y. Berest and A.P. Veselov. Hadamard's problem and Coxeter groups: new examples of Huygens' equations. Funkt. Anal. and applic., 28(1):3-15, 1994. russ.

[BV94b] Y.Y. Berest and A.P. Veselov. Huygens' principle and integrability (russ.). Uspechi mat. Nauk, 49(6):7-78, 1994.

[CCMW91] J. Carminati, S.R. Czapor, R.G. McLenaghan, and G.C. Williams. Consequences of the validity of Huygens' principle for the conformally invariant scalar wave equation, Weyl's neutrino equation and maxwell's equation on Petrov type II space-times. Ann. Inst. Henri Poincare, Phys. Theor., 54(1):9-16, 1991.

[CM86] J. Carminati and R.G. McLenaghan. An explicit determination of the petrov type $\mathrm{N}$ space-times on which the conformally invariant scalar wave equation satisfies Huygens' principle. Ann. Inst. Henri Poincare, Phys. Theor., 44:115-153, 1986.

[CM87] J. Carminati and R.G. McLenaghan. An explicit determination of the space-times on which the conformally invariant scalar wave equation satisfies Huygens' principle II. petrov type D space-times. Ann. Inst. Henri Poincare, Phys. Theor., 47:337-354, 1987.

[CM88] J. Carminati and R.G. McLenaghan. An explicit determination of the space-times on which the conformally invariant scalar wave equation satisfies Huygens' principle III. petrov type III space-times. Ann. Inst. Henri Poincare, Phys. Theor., 48:77-96, 1988.

[Feg84] H.D. Fegan. Differential equations on lie groups and tori, the wave equation and Huygens' principle. Rocky Mountain J. Math., 14:699-704, 1984.

[Fri75] F.G. Friedlander. The Wave Equation on a curved Space Time. Camb. Univ. Press, 1975. 
[Gün52] P. Günther. Zur Gültigkeit des Huygensschen Prinzips bei partiellen Differentialgleichungen vom normalen hyperbolischen Typus, volume 100(2) of Ber. Verh. Sächs. Akad. Wiss. Leipzig, Math.-Nat. Klasse. AkademieVerlag, Berlin, 1952.

[Gün65] P. Günther. Ein Beispiel einer nichttrivialen Huygensschen Differentialgleichung mit vier unabhängigen Veränderlichen. Archive Rat. Mech. and Analysis, 18:103-106, 1965.

[Gün88] P. Günther. Huygens' Principle and Hyperbolic Equations, volume 5 of Persp. in Math. Acad. Press Inc., Boston, 1988.

[GW85] P. Günther and V. Wünsch. On some polynomial conformal tensors. Math. Nachr., 124:217-238, 1985.

[GW86] P. Günther and V. Wünsch. Contributions to a theory of polynomial conformal tensors. Math. Nachr., 126:83-100, 1986.

[Had23] J. Hadamard. Lectures on Cauchy's Problem in Linear Partial Differential Equations. Yale Univ. Press, New Haven, 1923.

[Hel84] S. Helgason. Wave equation on homogeneous spaces. In Lie group representations, volume 1077 of Lecture Notes in Math., pages 254-287. SpringerVerlag, 1984.

[Hel92] S. Helgason. Huygens' principle for wave equations on symmetric spaces. J. Func. Anal., 107:279-288, 1992.

[He194] S. Helgason. Geometric Analysis on Symmetric Spaces, volume 39 of Mathematical Serveys and Monographs. AMS, Providence, Rhode Island, 1994.

[Ill88] R. Illge. On Huygens' principle for the relativistic higher spin wave equation of Buchdahl and Wünsch in presence of a gravitational and electromagnetic field. Math. Nachr., 139:237-243, 1988.

[McL69] R. McLenaghan. An explicit determination of the empty space-times on which the wave equation satisfies Huygens' principle. Proc. Cambr. Phil. Soc., 65:139-155, 1969.

[OS92] G. Olafsson and H. Schlichtkrull. Wave propagation on Riemannian symmetric spaces. J. Func. Anal., 107:270-278, 1992.

[Sch71] R. Schimming. Zur Gültigkeit des Huygensschen Prinzips bei einer speziellen Metrik. ZAMM, 51:201-208, 1971.

[Sch74] R. Schimming. Riemannsche Räume mit ebenfrontiger und ebener Symmetrie. Mathematische Nachrichten, 59:129-162, 1974. 
[Sch78] R. Schimming. Das Huygenssche Prinzip bei linearen hyperbolischen Differentialgleichungen 2. Ordnung für allgemeine Felder. Beiträge zur Analysis, 11:45-90, 1978.

[Sch84] R. Schimming. Konforminvarianten vom Gewicht -1 eines Zusammenhanges oder Eichfeldes. Zeitschrift für Analysis und Anwendungen, 3:401412,1984 .

[SS94] R. Schimming and H. Schlichtkrull. Helmholtz operators on harmonic manifolds. Acta Math, 173(2):235-258, 1994.

[Wün76] V. Wünsch. Über eine Klasse konforminvarianter Tensoren. Mathematische Nachrichten, 73:37-58, 1976.

[Wün78] V. Wünsch. Cauchy-Problem und Huygensches Prinzip bei einigen Klassen spinorieller Feldgleichungen I. Beiträge zur Analysis, 12:47-76, 1978.

[Wün79] V. Wünsch. Cauchy-Problem und Huygensches Prinzip bei einigen Klassen spinorieller Feldgleichungen II. Beiträge zur Analysis, 13:147-177, 1979.

[Wün80] V. Wünsch. Selbstadjungierte Huygensche Differentialgleichungen 2. Ordnung für nicht-skalare Spintensorfelder. Math. Nachr., 94:211-242, 1980.

[Wün85] V. Wünsch. Cauchy problem and Huygens' principle for relativistic higher spin wave equations in an arbitrary curved space-time. Gen. Relat. and Grav., 17:15-38, 1985.

[Wün89] V. Wünsch. Huygens' principle on Petrov type D space-times. Ann. der Physik, 46(8):593-597, 1989.

[Wün94] V. Wünsch. Moments and Huygens' principle for conformally invariant field equations in curved space-times. Ann. Henri Poincare Vol., 60(4):433$455,1994$.

Helga Baum

Ines Kath

Institut für Mathematik

Humboldt-Universität zu Berlin

Sitz: Ziegelstr. 13a

10099Berlin

baum@mathematik.hu-berlin.de kath@mathematik.hu-berlin.de 
Sfb 288 Preprints are availlable at:

http://www-sfb288. math.tu-berlin.de

List of most recent 50 sfb288 preprints

163 M. Karowski, R. Schrader: A lattice model of local algebras of observables and fields with braid group statistics

164 H. C. Hege, K. Polthier: Visualization and Mathematic

165 I. Krichever, A. Zabrodin: Spin generalisation of the RuijsenaarsSchneider model, non-abelian $2 \mathrm{D}$ Toda chain and representations of Sklyanin algebra

166 V. Bazhanov, A. Bobenko, N. Reshetikhin: Quantum Discrete Sine-Gordon Model at Roots of 1: Integrable Quantum System on the Integrable Classical Background

167 U. Bunke, M. Olbrich: Fuchsian groups of the second kind and representations carried by the limit set

168 H. Ferguson, A. Gray, St. Markvorsen: Costa's Minimal Surface via Mathematica

169 J. Dorfmeister, I. McIntosh, F. Pedit, H. Wu: on the Meromorphic Potential for a Harmonic Surface in a k-Symmetric space

170 M. Lüdde: Notes on generalised Magnus modules over the braid group

171 V. Bach, J. Fröhlich, I. M. Sigal: Mathematical Theory of NonRelativistic Matter and Radiation

172 P. Contucci, A. Knauf: The Phase Transition of the Number-Theoretical Spin Chain

173 K. Mohnke: On Seiberg-Witten Equations on Symplectic 4-manifolds

174 D. Ferus, F. Pedit: Curved Flats in Symmetric Spaces

175 M. Hinze: on the Numerical Approximation and Computation of MinimalSurface-Continua bounded by One-Parameter-Families of Polygonal Contours

$176 U$. Hertrich-Jeromin: on Conformally flat hypersurfaces, Curved flats and Cyclic sytems

177 U. Bunke, M. Olbrich: Cohomological properties of the smooth globalization of a Harish-Chandra module

178 F. Nill, K. Szlachányi: Quantum Chains of Hopf Algebras with Quantum Double Cosymmetry

179 St. Meißner, B.-D. Dörfel: Ground state and low excitations of an integrable chain with alternating spins

180 Ch. Bär: Harmonic Spinors for Twisted Dirac Operators

181 F. Constantinescu, M. Luedde: The Alexander and Jones-invariants and the Burau module

182 A. Bobenko, U. Eitner, A. Kitaev: Harmonic Inverse Mean Curvature Surfaces and Painleve Equations

183 K.-D. Kirchberg: Kählerian E-spinors

184 J. Kellendonk: Integer Groups of Coinvariants Associated to Octagonal Tilings

185 V. Kostrykin, R. Schrader: Ionization of Atoms and Molecules by Short strong Laser Pulses

186 M. J. Pflaum: A new concept of deformation quantization I. Normal order quantization on cotangent bundles 
188 U. Bunke, M. Olbrich: Cohomological properties of the canonical globalizations of Harish-Chandra modules

189 U. Bunke, M. Olbrich: Group cohomology and the singularities of the Selberg zeta function associated to a Kleinian group

190 H. Gollek: Deformations of isotropic curves in C3 and minimal surfaces in $\mathrm{R} 3$

191 F. Guerra, A. Knauf: Free Energy and Correlations of the NumberTheoretical Spin-Chain

192 H.-W. Wiesbrock: Symmetries and Modular Intersections of von-NeumannAlgebras

193 H.-W. Wiesbrock: Modular Intersections of von-Neumann-Algebras in Quantum Field Theory

194 H. Karcher, K. Polthier: Construction of Triply Periodic Minimal Surfaces

195 V. Bach, J. Poelchau: Accuracy of the Hartree-Fock Approximation for the Hubbard Model

196 F. V. Andreev, A. V. Kitaev: Connection Formulas for the asymptotics of the fifth Painleve transcendent on the real axis. II

197 J. Dorfmeister, G. Haak: On symmetries of constant mean curvature surfaces

198 Th. Friedrich: on Superminimal Surfaces

199 M. J. Pflaum: The normal symbol on Riemannian manifolds

200 M. U. Schmidt: On complex Bloch-spaces of periodic Schroedinger operators

201 A. Fring, V. Kostrykin, R. Schrader: On the absence of bound-state stabilization through short ultra-intense fields

202 C. Binnenhei: on The Even CAR Algebra

203 I. Kath: $G^{\star 2} 2(2)$-structures on pseudo-Riemannian manifolds

2.04 W. Müller, M. Schmidt, R. Schrader: Theta functions for infinite period matrices

205 V. M. Adamyan, H. Neidhardt: On the absolutely continuous subspace for contractions and dissipative operators

206 B. D. Dörfel, St. Meißner: Ground State structure and Low Temperature Behaviour of an Integrable chain with Alternating Spins

207 V. M. Adamyan, H. Neidhardt: On the absolutely continuous subspace for non-selfadjoint operators

208 V. Bach, J. Poelchau: Hartree-Fock Gibbs states for the Hubbard Model

209 Ch. Kreft, R. Seiler: Models of the Hofstadter Type

210 M. Lüdde: A Cellular Braid Action and the Yang-Baxter Equation

211 K. Große-Braukmann, K. Polthier: Constant Mean Curvature Surfaces Derived from Delaunay's and Wente's Examples

212 H. Baum, I. Kath: Normally hyperbolic operators, the Huygens property and conformal geometry 